\title{
Cooperation and competition in wild male Barbary macaques (Macaca sylvanus) in Morocco
}

\author{
Dissertation \\ for the award of the degree \\ “Doctor rerum naturalium" (Dr.rer.nat.) \\ of the Georg-August-Universität Göttingen
}

within the doctoral program Biology

of the Georg-August University School of Science (GAUSS)

\author{
Submitted by \\ Christopher Young \\ from Dundee, United Kingdom
}

Göttingen, 2013 


\section{$\underline{\text { Thesis Committee }}$}

Prof. Dr. Julia Ostner, Primate Social Evolution Group, Courant Research Centre Evolution of Social Behaviour

Prof. Dr. Peter Kappeler, Behavioral Ecology and Sociobiology Unit, German Primate Center $\mathrm{GmbH}$

\section{Members of the Examination Board}

Reviewer:

Prof. Dr. Julia Ostner

Second Reviewer:

Prof. Dr. Peter Kappeler

Further members of the Examination Board:

Dr. Antje Engelhardt, Jr Research Group on Primate Sexual Selection, Reproductive Biology Unit, German

Primate Centre $\mathrm{GmbH}$

Prof. Dr. Julia Fischer, Cognitive Ethology Lab, German Primate Center GmbH

Prof. Dr. Eckhard Heymann, Behavioral Ecology and Sociobiology Unit, German Primate Center $\mathrm{GmbH}$

Prof. Dr. Dirk Semmann, Evolution of Cooperation and Prosocial Behaviour Group, Courant Research Centre Evolution of Social Behaviour

Date of oral examination: 05.09.2013 




\section{Table of Contents}

Summary iii

Zusammenfassung....... vii

Chapter 1-General introduction 1

Chapter 2-Male mating behaviour in relation to female sexual swellings, socio-sexual behaviour and hormonal changes in wild Barbary macaques 17

With Bonaventura Majolo, Michael Heistermann, Oliver Schülke \& Julia Ostner

Chapter 3-Male coalitions and female behaviour affect male mating success independent of dominance rank and female receptive synchrony in wild Barbary macaques 37

With Sabine Hähndel, Bonaventura Majolo, Oliver Schülke \& Julia Ostner

Chapter 4-Beyond mutual tolerance - how males form coalitions against group rivals and the Pandit/van Schaik coalition model. 59

With Oliver Schülke \& Julia Ostner

Chapter 5 - Male social bonds predict partner choice in cooperative aggression in wild Barbary macaques. 79

With Bonaventura Majolo, Oliver Schülke \& Julia Ostner

Chapter 6-General discussion 101

References. 115

Acknowledgements 131

Cirriculum Vitae 


\section{Summary}

Mammalian males compete for a non-sharable resource (receptive females) and are typically the dispersing sex, thus cooperation between males may appear counterintuitive. However, if both partners gain mutual benefits from cooperating, such as an increase in mating/reproductive success, then cooperation can become a feasible strategy. Coalitions can be opportunistic, in the short-term providing direct benefits or can function to increase rank positions and provide future reproductive payoffs. These coalition types are predicted to occur at different levels of within group contest potential predicted by the monopolization potential of the alpha male over access to receptive females. It has been suggested males must weigh-up the rank/strength of the potential allies and the target to recruit a partner who will provide enough combined intrinsic fighting ability to defeat the target. Alternatively, males may base partner recruitment decisions on past experiences with group members (attitudinal partner choice) and regularly recruit reliable partners. Coalitionary partner choice may be mediated via social bonds under such circumstances.

The aim of this thesis was to shed light on male cooperation by examining male mating competition and social relationships and how these are influenced by coalitionary activity in a group living, wild, primate with male dispersal, the Barbary macaque (Macaca sylvanus). To this end I used a bottom-up approach, by examining the complete chain of events which led to malemale cooperation. I determined what males can ascertain about female reproductive state to gain an empirical estimate of mating contest potential within the groups. I utilised the Priority of Access model as a platform to investigate male mating competition and I examined the factors which led to deviation from the models predictions, namely female behaviour and male coalition formation. Species specific measures of male contest allowed for a critical evaluation of a mathematical model designed to predict different types of within group coalition formation (Pandit/van Schaik coalition model). Specifically, I examined how the model's predictions fitted to empirical data across two species with variable contest potentials (Barbary and Assamese macaques, $M$. assamensis). Male social relationships were analysed to determine if Barbary macaque males formed long-term social relationships, even during intense periods of competition (mating seasons). Finally, male social bond strength was examined to identify if they provided an adaptive benefit in terms of cooperation through coalition formation or if coalition partners were selected by hierarchal status alone. 
Data were collected on two groups of wild Barbary macaques in the Middle Atlas Mountains, Morocco (Sept. 2009-Aug. 2011). I collected over 2,000 hours of behavioural focal observations on all male subjects in the two groups as well as ad libitum data and 549 coalition bouts were observed of various size and success. Additionally, 155 female faecal samples were analysed to assess dates of conception using enzyme immune assays of progestogen metabolites, as well as visual monitoring of female sexual swelling size to determine receptive synchrony.

Analysis of female hormone concentrations revealed ovulation was most likely to occur during the maximum swelling period. However, male mating behaviour was further concentrated around the fertile phase implying that males infer information from more than swelling size alone. Male mating frequency increased in line with female socio-sexual behaviour. Most strikingly my results showed that males invested equally in mating during fertile and non-fertile, i.e. post-conception, maximum swelling phases. Despite these additional swelling periods reducing monopolisation potential of high ranked males mating was still skewed up the hierarchy but high ranked males did not gain as large a share as expected by the Priority of Access model. Females frequently initiated sexual encounters, predominantly with mid-ranked males, increasing their mating success while male coalitionary activity independently increased mating success. Frequent associations with females were costly to males as they were the targets of bridging coalitions, decreasing future mating opportunities for the targets. Highranking males did not increase their mating success directly through bridging coalitions but acted to dilute the effects of female mating behaviour. Furthermore, Barbary macaque males formed long-term social bonds enduring through highly competitive mating seasons. Males who shared strong social bonds were more likely to be recruited as coalition partners suggesting that males use more than merely rank position of available partners when recruiting. Examination of the Pandit/van Schaik coalition model showed that at high contest potential opportunistic coalitions should be utilized to gain access to females monopolised by high ranked males. Whereas the empirical data did not match the predictions at mid-low contest potential where male affiliation may play a role in facilitating rank-changing coalitions which require reliable partners to defend higher rank positions once obtained. Thus, strong social bonds can provide both short- and long-term benefits by reducing the likelihood of partner defection during coalitions and providing a long-term partner to facilitate successful rank-changes and ultimately increase both status and mating/reproductive success.

In sum, my thesis adds to the current literature on the links between male reproductive strategies, social bonding and cooperation in group living mammalian societies, with male dispersal. Through investigation of the complete chain of evidence from male contest 
competition to mating success and cooperation via male social bonding, this study united several previously separate pieces of research into one comprehensive picture and may provide a template for future research. Coalitionary activity can provide mutual benefits for both partners suggesting that cooperation may be based on previous affiliative interactions with group members brought about by attitudinal partner choice. 


\section{Zusammenfassung}

Männliche Säugetiere konkurrieren um eine nicht-teilbare Ressource (empfängnisbereite Weibchen) und sind üblicherweise das abwandernde Geschlecht, weshalb Kooperation zwischen Männchen der Intuition zu widersprechen scheint. Wenn die Kooperation jedoch für beide Partner vorteilhaft ist, indem sich z.B. ihr Paarungs-/Fortpflanzungserfolg erhöht, kann Kooperation eine plausible Strategie sein. Koalitionen können opportunistisch sein, also kurzfristig einen direkten Nutzen bieten, oder für einen zukünftigen Fortpflanzungserfolg und Aufstieg in der Rangordnung sorgen. Welche Art von Koalition auftritt, hängt von dem Konkurrenzpotential innerhalb einer Gruppe ab, das von der Fähigkeit des alpha-Männchen, rezeptive Weibchen zu monopolisieren, bestimmt wird. Es wird davon ausgegangen, dass Männchen den Rang bzw. die Stärke von potentiellen Koalitionspartnern und Kontrahenten abwägen müssen, um einen Partner zu rekrutieren, so dass die gemeinsame intrinsische Kampfkraft ausreicht um den Kontrahenten zu besiegen. Alternativ können Männchen vorherige Erfahrungen mit Gruppenmitgliedern nutzen, um zu entscheiden wen sie rekrutieren ("attitudinal partner choice") und verlässliche Partner wählen. Unter solchen Bedingungen kann die Wahl des Koalitionspartners durch Sozialbeziehungen beeinflusst werden.

Diese Doktorarbeit sollte Aufschluss geben über die Kooperation zwischen Männchen, indem die Paarungskonkurrenz und Sozialbeziehungen von Männchen und der Einfluss von Koalitionen in einer gruppenlebenden, wilden Primatenart, dem Berberaffen (Macaca sylvanus), untersucht wurden. Dazu habe ich einen Bottom-Up-Ansatz genutzt, indem ich die vollständige Folge von Ereignissen analysierte, die zu Kooperation zwischen Männchen führte. Ich habe bestimmt, inwiefern Männchen den reproduktiven Zustand von Weibchen feststellen können, um das Potential zur Paarungskonkurrenz innerhalb von Gruppen empirisch abschätzen zu können. Ich habe das "Priority-of-Access"-Modell als Grundlage für eine Erforschung der männlichen Paarungskonkurrenz genutzt und habe die Faktoren untersucht, die zu Abweichungen von den Vorhersagen des Modells führen, vornehmlich das Verhalten der Weibchen und die männliche Koalitionsbildung. Die artspezifische Messung der Konkurrenz zwischen Männchen erlaubte eine kritische Evaluation eines mathematischen Modells, das konzipiert wurde, um verschiedene Typen von Koalitionsbildung innerhalb von Gruppen vorherzusagen (Pandit/van SchaikKoalitionsmodell). Im Besonderen habe ich untersucht, wie die Vorhersagen des Modells zu empirischen Daten zweier Arten mit variablem Konkurrenzpotential (Berberaffen und AssamMakaken, M. assamensis) passen. Die Sozialbeziehungen von Männchen wurden analysiert, um 
festzustellen, ob Berberaffen selbst in Zeiten intensiver Konkurrenz (während der Paarungszeit) langfristige Beziehungen formen. Schlussendlich wurde die Stärke männlicher Sozialbeziehungen untersucht um zu ermitteln, ob sie einen Anpassungswert durch die Kooperation in der Koalitionsbildung bieten, oder ob Koalitionspartner allein aufgrund ihres Dominanzstatus ausgewählt wurden.

Es wurden Daten zweier Gruppen freilebender Berberaffen im Mittleren Atlasgebirge Marokkos von September 2009 bis August 2011 gesammelt. Ich habe über 2000 Stunden Verhaltensdaten aller Männchen in beiden Gruppen nach der Focus-Tier-Methode sowie ad libitum gesammelt, und 549 Koalitionen verschiedener Größe und unterschiedlichen Erfolges beobachtet. Zusätzlich wurden 155 weibliche Kotproben analysiert, um das Datum der Empfängnis mittels Enzymimmunassays von Gestagenmetaboliten festzustellen. Außerdem wurden weibliche Sexualschwellungen visuell eingestuft, um die Synchronität der Empfängnisse zu ermitteln.

Die Analyse der weiblichen Hormonkonzentrationen zeigt, dass die Ovulation am wahrscheinlichsten im Zeitraum maximaler Sexualschwellung standfand. Allerdings konzentrierte sich das männliche Paarungsverhalten stärker auf die fertile Phase, was impliziert, das Männchen mehr als nur die Schwellungen an sich als Information nutzen können. Die Paarungshäufigkeit der Männchen stieg im Einklang mit dem sexualen Sozialverhalten der Weibchen. Bemerkenswerterweise zeigen meine Ergebnisse, dass Männchen gleichermaßen in Paarungen während der fertilen und nicht-fertilen (d.h. nach der Empfängnis) Phasen der Sexualschwellungen investieren. Obwohl diese zusätzlichen Sexualschwellungen das Monopolisierungspotential hochrangiger Männchen verringern, waren Paarungen zu Gunsten hochrangiger Männchen verschoben. Hochrangige Männchen haben aber keinen so großen Anteil der Paarungen erzielt, wie vom "Priority-of-Access"-Modell vorhergesagt wird. Weibchen haben regelmäßig Paarungen initiiert, in erster Linie mit mittelrangigen Männchen, was deren Paarungserfolg erhöhte, während Koaltionsbildung von Männchen deren Paarungserfolg unabhängig davon erhöhte. Regelmäßige Assoziationen mit Weibchen waren kostspielig für Männchen, da sie Ziele überbrückender Koalitionen ("bridging coalitions") wurden, was ihre zukünftigen Paarungsmöglichkeiten einschränkte. Hochrangige Männchen haben ihren Paarungserfolg nicht direkt mittels überbrückender Koalitionen ("bridging coalitions") erhöht, minderten aber den Einfluss des weiblichen Verhaltens. Außerdem bildeten Berberaffenmännchen langfristige Sozialbeziehungen die durch die höchst kompetitiven Paarungzeiten hindurch bestehen blieben. Männchen mit einer starken sozialen Bindung wurden mit höherer Wahrscheinlichkeit als Koalitionspartner rekrutiert, was nahe legt, dass für 
Männchen mehr als nur der Rang der verfügbaren Partner ausschlaggebend ist. Eine Prüfung des Pandit/van Schaik-Koalitionsmodells zeigte, dass unter hohem Konkurrenzpotential opportunistische Koalitionen genutzt werden sollten, um Zugang zu Weibchen zu gewinnen, die von hochrangigen Männchen monopolisiert sind. Indessen stimmten die empirischen Daten nicht mit den Vorhersagen unter mittlerem bis niedrigem Konkurrenzpotentials überein, bei dem männliche Affiliationen Rang-verändernde Koalitionen erleichtern könnten. Für diese werden vertrauenswürdige Partner benötigt, um die höherrangige Position zu verteidigen, sobald sie gewonnen wurde. Demnach können enge soziale Bindungen zwischen Männchen sowohl kurzals auch langfristige Vorteile liefern, indem die Wahrscheinlichkeit, dass der Partner während einer Koalition abtrünnig wird, verringert wird. Zudem steht ein langfristiger Partner zur Verfügung, der erfolgreiche Rangveränderungen erleichtert wodurch enge soziale Bindungen letzlich sowohl den Status als auch den Paarungs-/Fortpflanzungserfolg erhöhen.

Zusammenfassend erweitert meine Doktorarbeit die derzeitige Literatur über den Zusammenhang zwischen männlichen Fortpflanzungsstrategien, Sozialbeziehungen und Kooperation innerhalb von gruppenlebenden Säugetieren mit abwandernden Männchen. Durch die Untersuchung der vollständigen Beweiskette vom zwischenmännlichem Konkurrenzkampf hin zu Paarungserfolg und Kooperation durch das Schließen enger zwischenmännlicher Sozialbindungen, ergibt diese Studie ein umfassendes Bild mehrerer unabhängiger Forschungsansätze und könnte eine Vorlage für zukünftige Forschungsarbeiten bilden. Koalitionsbildung kann mutualistische Vorteile für beide Partner liefern, was nahelegt, dass Kooperation durch vorherige affiliative Interaktionen mit Gruppenmitgliedern durch "attitudinal partner choice" bestimmt sein könnte. 


\section{Chapter 1}

\section{General Introduction}

Cooperation in animal societies by definition must carry costs for at least one of the participants and therefore for cooperation to evolve costs should be offset by benefits (Hamilton 1964a; 1964b; Trivers 1971). Cooperation in eusocial insects and cooperatively breeding birds and mammals has been the subject of much theoretical and practical research (reviewed by CluttonBrock 2009b; Lukas and Clutton-Brock 2012). Inclusive fitness benefits are posited to explain the evolution of cooperation in these species (reviewed by Clutton-Brock 2009b; Lukas and CluttonBrock 2012; but see Nowak et al. 2011). Similarly, inclusive fitness benefits are also considered to be the main evolutionary driving force behind female cooperation in multi-male, multi-female group living mammalian societies (reviewed by Silk 2007; Clutton-Brock and Lukas 2011) as females are usually the philopatric sex (Johnson 1986). Recent studies have begun to reveal the fitness benefits of cooperation for mammalian females. However, there are still sizable gaps in our understanding of male-male cooperation as males often disperse from their natal groups and compete over non-sharable resources, namely females (van Hooff and van Schaik 1994). Here, I aim to shed light on male cooperation examining male mating competition and social relationships and how these are influenced by coalitionary activity in a group living, wild, social mammal with male dispersal, the Barbary macaque (Macaca sylvanus).

In the following general introduction I will outline the current knowledge on the evolution of cooperation in animal societies (section 1.1), before exploring how within group cooperation and social relationships have evolved in mammalian societies between both kin and unrelated individuals (section 1.2). Further, I describe how male competition, and thus cooperation, within groups is driven by male contest for access to receptive females (section 1.3). I will then introduce and examine coalition formation, a cooperative act observed in many non-human primate and some mammalian societies (section 1.4). In the following section I will tie these topics together by explaining how male social relationships, contest competition and cooperation through coalition formation are linked (section 1.5). I then briefly describe my study species, the Barbary macaque, the field site and current relevant knowledge on the species (section 1.6), before outlining the aims of the thesis in section 1.7. 


\subsection{Cooperation in animal societies}

In multi-male, multi-female animal societies, where several individuals live in groups, cooperation is thought to evolve between individuals if it provides fitness benefits for both participants. Cooperation between kin has been explained through inclusive fitness benefits and individuals aiding relatives gaining benefit by passing on part of their genetic make-up through their kin (Hamilton 1963; 1964a; 1964b). Cooperation between non-kin is more puzzling as individuals increase the benefits of their partner whilst cooperation comes at some form of cost to themselves, such as increased risk of injury or a reduction in time for other activities and allows for cheating (see below) (West et al. 2007b; Clutton-Brock 2009a; Gilby 2012). Cooperation in animal societies can often be between non-kin (reviewed in Clutton-Brock 2009a; Melis and Semmann 2010) and thus, several alternative mechanisms have been proposed to explain the evolution of cooperation (reviewed by Dugatkin 1997; Nowak 2006; West et al. 2007b; Clutton-Brock 2009a).

Cooperation between non-kin can arise if both partners gain direct benefits or the exchange between partners should be equal and a cooperative act received should be followed by a cooperative act given in the future, reciprocal altruism (Trivers 1971). An individual's desire to gain short-term benefits through defecting should be out-weighed by longer-term benefits of cooperation and thus, should be more likely to develop in social group living species where the opportunity to reciprocate is enhanced compared to solitary or pair-living species (Trivers 1971). However, empirical support for reciprocal altruism in animal societies is rare due to the proposed inflexibility of cooperative exchange (West et al. 2007b; Clutton-Brock 2009a; but see Schino and Aureli 2010). Cooperation between non-kin may provide mutual benefits (as defined by West et al. 2007a) for both partners and thus, the need for complete reciprocation is not required as partners always gain benefits (West et al. 2007b; Clutton-Brock 2009a). Cooperation in stable groups should allow for contingent exchange of commodities and partner choice in which cooperation depends on either previous or future cooperation (Nowak 2006; CluttonBrock 2009a; Gilby 2012).

Biological market theory (Noë et al. 1991; Noë and Hammerstein 1994; 1995; reviewed by Barclay 2013) posits that, within groups, individuals each have commodities which they can then trade with each other for the same or different commodities. For example, in primate societies this could be grooming, agonistic support or tolerance at feeding sites (Barrett et al. 1999). In these exchanges, a certain level of trust must be present or one individual (for example the individual first receiving grooming) could then defect when their partner requires to "cash in" their trade (support in agonistic aggression). It may be that these short-term (or long-term in a 
stable environment) exchanges and fluctuations in the value of commodities can better account for the observed patterns of cooperation allowing for asymmetries in exchange (Henzi and Barrett 1999; Barrett and Henzi 2001; 2006; but see Schino and Aureli 2009) rather than the equitable exchanges of reciprocal altruism (Trivers 1971).

Brosnan and de Waal (2002; also see de Waal 2000) proposed two mechanisms, calculated (cognitively based) and attitudinal (emotionally based) reciprocity, which may explain the underlying mechanisms of partner choice. Calculated reciprocity would require advanced cognitive capabilities for individuals to plan future interactions with group members in order to gain future benefits which may be beyond many non-human primates (Stevens and Hauser 2004; Stevens et al. 2005; but see Dufour et al. 2009; reviewed by Schino and Aureli 2010). Alternatively, attitudinal reciprocity is dependent on the social relationships between individuals. Group members make decisions on the partners they choose to cooperate with based on previous experiences/interactions and selection of the best partner for the task available, leading to "attitudinal partner choice" (Noë and Völkl 2013). Perhaps less cognitively demanding and within the capacities of many non-human primates (Schino and Aureli 2010). This concept builds on the idea of emotional bookkeeping (Schino and Aureli 2009), a mechanism whereby individuals keep track of previous interactions in a "bookkeeping" format driven by an emotional state towards other group members based to previous experiences. Using this mechanism, individuals need not keep track of the magnitude of services exchanged but merely update their emotional relation to the partner and base their decisions to cooperate on this cognitively less taxing form of emotional bookkeeping. In species where social relationships show differentiation within groups attitudinal partner choice may act to foster cooperation between individuals based on their previous experiences.

\subsection{Within group social bonding and cooperation}

Individuals living in groups experience both negative and positive interactions with other group members and recurring affiliative/positive interactions between individuals leads to the development of social relationships (Hinde 1976; 1983). Social relationships are considered to develop into social bonds over time, if the social relationship is stable, relatively balanced in directionality between the partners and strong compared to others within the group (Silk 2002c). In some species affiliative behaviour can merely equate to siting within close proximity or body contact without receiving aggression (Wey and Blumstein 2012). Whereas other species can show varied and more elaborate social behaviours such as ravens, Corvus corax, (preening behaviour; Fraser and Bugnyar 2010; Braun and Bugnyar 2012) and non-human primates where social behaviour such as grooming amounts to a large part of their daily activity budget (Dunbar 
1991). Engaging in social behaviour can be costly, for instance extended grooming bouts for longer than would be required for hygienic purposes can lead to reduced feeding time or mating opportunities (Dunbar 1991). Thus, maintenance of social bonds can take time away from other daily requirements and therefore must provide an adaptive benefit to have developed (reviewed by Silk 2002c; 2007; 2012).

Whether repeated interactions between two individuals can be considered a social bond however has been questioned (Barrett et al. 2007). In stable groups, such as primates, available partners and quality of partners may not change dramatically over extended time periods and thus, individuals may consistently select the same partner to meet their current needs. If there is external instability, such as food shortages, then individuals may be more inclined to change their partner preferences and select new preferred partners afterwards (Henzi et al. 2009). However, a recent study on Barbary macaques strongly suggests the presence of social bonds between males (Berghänel et al. 2011a). The frequent affiliation between dyads before the mating season predicted the frequency with which males formed coalitions in the mating season while controlling for affiliation rates within the mating season. This implies that these males may call upon some previous knowledge acquired before the mating season when supporting in agonistic aggression. Additionally, Crockford et al. (2013) found in wild chimpanzees, Pan troglodytes, that oxytocin levels (a hormone related to bond formation and maintenance) showed a significant increase only between strongly bonded dyads during grooming bouts. If grooming dyads were not strongly bonded no rise in oxytocin levels was observed, suggesting that individuals may have some representation of their social relationships with partners (Crockford et al. 2013).

Mammalian females usually associate with close kin and thus, stronger relationships form between kin, although strong relationships between non-kin are not uncommon (Silk 2002a). It has been observed across several taxa that individuals who form stronger social bonds in groups can gain reproductive benefits (Armitage and Schwartz 2000; Connor et al. 2001; Frère et al. 2010; Wey and Blumstein 2012). Longitudinal studies on baboon groups have shown that females form long-term, stable and enduring social bonds with group mates (Silk et al. 2006a; 2006b; 2010a). These bonds are highly differentiated within the group and those females with strong bonds tend to reap many benefits such as increased infant survival (Silk 2003; Silk et al. 2009). If one member of a strongly bonded dyad dies the partner's stress levels rise significantly (Engh et al. 2006). Individuals with more focused social networks were also shown to experience lower stress levels (Crockford et al. 2008; Wittig et al. 2008). Social bonds have also been shown in humans and non-human primates to increase survival and reduce mortality risks (Holt-Lunstad 
et al. 2010). Additionally, male-female dyads which exhibit strong social bonds are known to increase the reproductive success of the male and provide the female with future protection of her infant (Palombit et al. 1997; 2000; Weingrill 2000), reduce female stress levels during alpha male takeovers (Beehner et al. 2005) or increase overall reproductive success (Weidt et al. 2008; Langergraber et al. 2013).

Male dispersal and intense competition between males over access to females led researchers to propose that cooperation between males was less likely than between females (van Hooff and van Schaik 1994). However, recent evidence suggests that affiliation between male primates is more wide spread than originally thought (Silk 1994; Perry 1998; Connor et al. 2001; Duffy et al. 2007; Mitani 2009; Fraser and Bugnyar 2010; Schülke et al. 2010; Berghänel et al. 2011a; Teichroeb et al. 2013; Xia et al. 2013; Ostner and Schülke submitted). In chimpanzees where males remain in their natal groups, long-term affiliative relationships often develop between non-kin, suggesting that even when available relatedness may not be a prerequisite for social bond formation (Goldberg and Wrangham 1997; Mitani et al. 2000; Vigilant et al. 2001; Langergraber et al. 2007). These bonds in chimpanzees can even match the patterns found between philopatric female baboons (Silk et al. 2006a; 2006b; 2010a) in their stability, equability and endurance (Mitani 2009). Male dyads which form strong bonds are more likely to cooperate, for example, in coalitionary support (Silk 1994; Connor et al. 2001; Mitani et al. 2002; Watts 2002; Perry et al. 2004; Schülke et al. 2010; Berghänel et al. 2011a; Gilby et al. 2012), boundary patrols (Boesch and Boesch-Achermann 2000; Mitani 2006a), cooperative hunting (Boesch 1994a; Boesch 1994b). Additionally, comparable to female-female social bonds, a growing body of literature suggests that cooperating males gain fitness benefits over other group rivals (Duffy et al. 2007; Schülke et al. 2010; Gilby et al. 2012).

\subsection{Male Contest}

Cooperation between males is predicted to evolve differently under varying levels of contest (van Hooff and van Schaik 1992; Pandit and van Schaik 2003) and expected to be absent at extremes of high and low contest. Thus, we first need to understand what males are competing for and the level of contest competition, in order to examine male-male cooperation. Over time, repeated agonistic interactions between males will lead to the formation of a dominance hierarchy based on fighting abilities of individuals in the group (Dewsbury 1982; Drews 1993; Watts 2010). The males with the highest dominance ranks are expected to gain priority of access to resources and gain benefits befitting of their rank position. The ultimate resource males compete for is access to receptive females and high ranked males can monopolise access depending on the number of synchronously receptive females, conceptualised in the Priority of 
Access model (hereafter the PoA model; Altmann 1962). As more females become receptive synchronously, the alpha male can only defend temporal access to one female and access to other females is determined on a hierarchal basis. Several comparative analyses across primate species have shown the potential for the alpha male's mating/reproductive skew to be predicted by the level of female reproductive synchrony (Kutsukake and Nunn 2006; Ostner et al. 2008b; Gogarten and Koenig 2012). Thus, female reproductive synchrony plays an important role in male mating/reproductive success in many multi-male, multi-female societies. Therefore, the PoA model may provide an ideal platform to examine male mating success, in comparison to many other reproductive skew models which do not incorporate female reproductive synchrony into their design (reviewed in Port and Kappeler 2010).

The degree to which the alpha male can monopolise access to females can be used to provide an estimate of the contest potential within groups, using the predicted alpha male mating/reproductive success of the PoA model (Pandit and van Schaik 2003; van Schaik et al. 2004a; 2006). However, the extent to which males can ascertain female receptive synchrony varies between species in many non-human primates (reviewed in Dixson 2012). Thus, gaining an accurate estimate of the information males can infer about female reproductive state is vital in order to accurately estimate contest competition (Alberts et al. 2003; Wroblewski et al. 2009; Henzi et al. 2010). Males and females face a continual arms-race in terms of competing reproductive strategies (Trivers 1972). Females living in multi-male, multi-female groups face a quandary, termed the "female dilemma" (van Schaik et al. 2000). Females should give all males a non-zero probability of paternity, to reduce future infanticide risk, and concentrate mating to the male with the "best" genes around the period of fertility (van Schaik et al. 2000). Many species of primate show extended periods of sexual receptivity and mate extensively beyond the period when conception may occur (reviewed by Hrdy and Whitten 1987; Dixson 2012) and even into gestation (Ostner et al. 2006; Engelhardt et al. 2007; Fürtbauer et al. 2011a; Lu et al. 2012) to attempt to solve this dilemma.

Exaggerated sexual swellings have developed across many female primates, particularly in, but not limited to, cercopithecines and apes (reviewed by Nunn 1999a). The swelling increases in size reaching maximum size around the time of ovulation and rapidly deflates after ovulation, providing a probabilistic cue to males as to when ovulation will occur (Dixson 1998; Nunn 1999a; Nunn et al. 2001). In addition to maximum swelling size males can ascertain additional information about female reproductive state from more subtle changes in swelling size (Deschner et al. 2004; Higham et al. 2009), olfactory cues around ovulation (Cerda-Molina et al. 2006) or female proceptive behaviour (Wallen et al. 1984; Aujard et al. 1998; Zehr et al. 1998; 
2000; Engelhardt et al. 2005). The extent to which males can do so varies between species however, and some species have even been shown to produce additional swellings during gestation (Gordon et al. 1991; Gust 1994; Engelhardt et al. 2007) which may serve as an additional mechanism to further confuse paternity (van Schaik et al. 2000; 2004b). These postconception swelling periods and related female behaviours have been reported in the study species of this thesis, the Barbary macaque (Kuester and Paul 1984; Möhle et al. 2005; Pfefferle et al. 2011).

Male reproductive strategies can only evolve in relation to the information that is available to males. If the alpha male is unable to infer when ovulation is most likely, estimates of contest potential within these species need to be adjusted to account for this (Alberts et al. 2003; Wroblewski et al. 2009; Henzi et al. 2010). If receptivity is extended or ovulation concealed then mating will not be concentrated solely around the fertile period and offspring could be sired by males by chance. This would lead to inaccurate estimates of the alpha male's monopolisation potential and thus estimates of contest potential derived from the PoA model predictions. The information males can infer about female reproductive state will define the within group contest competition. Therefore, empirical estimates of the information males can infer about female reproductive state are critical before examining mating competition via the PoA model (see Chapter 2). Only once these accurate estimates of contest competition are known can predictions of the types of alternative mating strategies likely to occur between males be estimated, such as coalitionary aggression.

\subsection{Cooperation through coalition formation}

Coalitionary cooperation has been observed in a wide variety of taxa from birds (Loretto et al. 2012) to cetaceans (Connor et al. 1992) to social carnivores (de Villiers et al. 2003; Smith et al. 2010) to many non-human primate species (reviewed in Smith et al. 2010) and many human societies (Boehm 1999; Bowles 2007). Coalitions can provide many different functions including defence of or access to resources, rise in rank position for one or both partners, defence against aggression from a more dominant individual or defence against group take-over from rivals (reviewed in Harcourt and de Waal 1992; Olson and Blumstein 2009; Smith et al. 2010). For group-living females coalition formation has largely been attributed to nepotism and kin-related benefits of cooperating and supporting relatives (Wrangham 1980; van Schaik 1989; Isbell 1991; Sterck et al. 1997). Female group demographics therefore form the base of many theoretical and mathematical models designed to investigate different aspects of coalition formation. For example, examining relatedness (Broom et al. 2009) or access to food resources (Stamatopoulos et al. 2009). Furthermore, models have looked at various additional factors from the influence of 
encounter frequency on the tendency to cooperate in fission-fusion societies (Connor and Whitehead 2005), the motivation to interfere in on-going dyadic contests (Dugatkin 1998; Johnstone and Dugatkin 2000) to optimal size models (Whitehead and Connor 2005). Mammalian males are often the dispersing sex (Johnson 1986) and compete over a non-divisible resource (van Hooff and van Schaik 1994). Therefore, these female-based models which assume philopatric female group members or competition for divisible resources are problematic to apply to males. Other proposed models have examined male coalition formation, for example, payoff structures between allies (Noë 1990), the combined fighting abilities of allies (Noë 1994) but have focused only on one coalition type (e.g. within group all-up levelling coalitions, see below).

Recently a mathematical model has been developed to explain the evolution of different malemale coalition types within groups (Pandit and van Schaik 2003; van Schaik et al. 2004a; 2006). This model was designed to evaluate the specific circumstances under which different coalition types are likely to evolve in primate groups. I evaluate the Pandit/van Schaik coalition model (hereafter PvS model) in detail in Chapter 4 nevertheless; I provide a brief summary of the expectations of the model here. The model assesses the profitability (the coalition must provide fitness benefits for the partners) and feasibility (the coalition partners must be able to defeat the target) of certain coalitions occurring at different levels of contest competition within groups (Pandit and van Schaik 2003; van Schaik et al. 2004a; 2006).

Following Chapais (1995), Pandit and van Schaik (2003) classified coalitions into three constellations based on the ranks of the allies and target in the coalition: 1) all-up, where both allies are lower ranking that the target, 2) all-down, where both allies are higher ranking than the target, and 3) bridging, where the target is ranked between the allies. These coalition constellations were further categorized into types by their function, being either 'rank-changing' when they affect participants' rank position, or 'levelling', when they reduce the inequality in the distribution of matings/paternity across ranks (van Schaik et al. 2004a). One of the main assumptions of the model is that payoffs for the coalition partners are mediated by dominance rank rather than male fighting abilities. The PoA model (Altmann 1962) was utilized by Pandit and van Schaik (2003) to assign the different payoffs for each ally depending on their rank (see Chapter 3 for details of the PoA model). The parameter " $\beta$ " in the model was derived from the PoA model as an estimate of contest within the group by estimating the skewedness of mating and thus, the payoff curve by dominance rank. Accordingly, $\beta$ is equal to the degree of within group contest between males for access to receptive females. In the model the $\beta$ parameter best explained which coalition constellations and types were most likely to occur under different 
circumstances. In order to examine the PvS model and the coalitions being formed within groups an accurate measure of male contest potential is required. Therefore, an accurate test of the PoA model must be based on the species specific information available to males about female reproductive state, see above (Alberts et al. 2006; Wroblewski et al. 2009; Henzi et al. 2010).

Within groups, males can cooperate opportunistically to gain access to receptive females, forming levelling coalitions (Pandit and van Schaik 2003). Males can team up to break up a consortship between a male and female dyad to gain access to the receptive female. As has been observed in baboons (Packer 1979; Bercovitch 1988; Noë and Sluijter 1990; 1995) and Barbary macaques (Bissonnette et al. 2011), effectively creating a level mating skew for the lower ranked males. By combining their intrinsic fighting abilities males are able to reduce mating opportunities of higher ranked rivals. However, only one ally can then gain access to the female, leaving an uneven distribution of payoffs. The partner gaining access to the consorted female is not always the highest ranked coalition partner (Bercovitch 1988; Noë 1992; Bissonnette et al. 2011) suggesting that both partners may be able to reap mating benefits from levelling coalitions over a longer time period. Levelling coalitions provide a short-term, opportunistic alternative mating strategy to gain direct benefits in the form of access to consorted females. However, males can also adopt longer-term reproductive strategies and cooperate with a partner over time to raise their rank position and gain hierarchal reproductive benefits.

\subsection{Male coalitions, social bonds and cooperation}

Coalition formation is not only beneficial in terms of gaining temporal access to females and the payoffs to individuals need not only concern immediate benefits of access to the female. Male rank position has been found to be highly correlated to reproductive success in comparative analysis (Cowlishaw and Dunbar 1991; Ellis 1995; Majolo et al. 2012). Thus, attaining high rank position can increase a male's reproductive success. Males can cooperate through rank-changing coalitions to increase the rank position of one or both partners (van Schaik et al. 2004a; 2006). Rank changes through coalition formation can be achieved with one interaction but may require repeated coalitions afterwards to defend the new rank position and thus, partners may need to interact over longer periods than with levelling coalitions. Hence, these coalitions require the partners to form repeated coalitions over time to eventually reap the long-term benefits (van Schaik et al. 2006; Ostner and Schülke submitted).

Attempting to increase rank position through coalition formation is a risky behaviour and therefore requires "trust" between the two partners so that one partner does not defect and 
leave its partner in a dyadic contest (van Schaik et al. 2006; Clutton-Brock 2009a). The formation of social bonds between group members may help to facilitate this cooperation between individuals. Repeated affiliation between individuals through social behaviours such as grooming, being in close proximity without aggression or triadic male-infant-male interactions may show a willingness to cooperate and develop strong social bonds between individuals (Ostner and Schülke submitted). As stated above the correlation between cooperation through coalition formation and male affiliative behaviour is becoming more apparent (Silk 1994; Watts 2002; Perry et al. 2004; Duffy et al. 2007; Schülke et al. 2010; Berghänel et al. 2011a; Gilby et al. 2012; Ostner and Schülke submitted). Additionally, a correlation between grooming given and agonistic support was found in a comparative analysis across several primate species in both sexes (Schino 2007). The frequency with which male Assamese macaques (Macaca assamensis) formed coalitions predicted their future rank position, with those forming coalitions more often rising in rank in the future (Schülke et al. 2010). This study was able to show a chain of evidence that males who were strongly bonded, frequently formed coalitions, attained higher future rank position and ultimately increased reproductive success (Schülke et al. 2010). Conversely, those males without strong bonds, formed coalitions less frequently and fell in rank in the future and males' coalition partner selection was based on bond strength and not the current rank position of partners.

Similarly, male chimpanzees form strong bonds with both kin and non-kin (Mitani et al. 2002; Langergraber et al. 2007; Mitani 2009) and males frequently exchange grooming for support in agonistic aggression (Watts 2002). Coalitionary support of the alpha male can also translate into mating concession by the alpha male for subordinates (Duffy et al. 2007). Furthermore, social network analysis of coalition networks between males found individuals with high centrality rose in rank and had higher reproductive success than similarly ranked group mates with lower centrality scores. Thus, coalitionary activity provided both short- and long-term reproductive benefits for chimpanzee males (Gilby et al. 2012). These studies in both macaques and chimpanzees highlight the potential for longer-term benefits of coalitionary support. In species where mating and reproduction is not highly skewed towards the alpha male (but the alpha still gains the highest proportion of mating success) the formation of social bonds through long-term affiliation can lead to cooperation in coalition formation and future reproductive success. This connection between male contest potential leading to mating skew, cooperation and social relationships was what I set out to examine in this thesis. 


\subsection{Study site and species}

In this thesis I examined social and sexual behaviour to determine the effect of male contest competition and male social bond strength on coalitionary aggression in wild, male Barbary macaques. The study was conducted at the field site of the Barbary Macaque Project, a collaboration between Dr. Bonaventura Majolo (University of Lincoln, U.K.) and Prof. Mohamed Qarro (École Nationale Forestière d'Ingénieurs, Salé, Morocco), a longitudinal project examining the socioecology of wild Barbary macaques since 2008 in the Middle Atlas Mountains, Morocco $\left(33^{\circ} 24^{\prime} 9 \mathrm{~N}-005^{\circ} 12^{\prime} 9 \mathrm{~W}\right.$; Majolo et al. 2013).

Barbary macaques are a member of the Macaca genus and are considered the phylogenetic ancestral species of all macaques (Purvis 1995; Morales and Melnick 1998). Wild populations of Barbary macaques are found in both Algeria and Morocco as well as a free-ranging population in Gibraltar, U.K. (Fooden 2007). The species is characterised by a multi-male, multi-female social organisation with a promiscuous mating system whereby both males and females mate with many partners (Taub 1980; Small 1990; Kuester and Paul 1992). The species reproduces seasonally with a short mating season from September to January, followed by a birth season from March to June (Taub 1980; Kuester and Paul 1992; Menard and Vallet 1996; Brauch et al. 2007). This provides periods of intense competition between males for access to receptive females in the mating season and can lead to high levels of female receptive synchrony (Möhle et al. 2005). Females show exaggerated sexual swellings which reach maximum size around ovulation when males concentrate their mating behaviour (Brauch et al. 2007; Heistermann et al. 2008). Females also produce an anogenital sexual swelling during gestation although whether males can distinguish between these swellings and those which occur during ovulation was unknown prior to this study (Möhle et al. 2005).

A previous study under free-ranging conditions at Affenberg Salem, Germany, examined factors driving male mating success (Bissonnette et al. 2011) and found a poor fit of the observed mating skew to the PoA model. Mating success of low-ranked, post-prime males was increased via coalitionary activity where they broke up consortships of high-ranked males utilizing all-up levelling coalitions. This coalitionary activity may have been counteracted by females initiating consortships with higher ranked prime-males. In this provisioned group, male and female group size was largely inflated and age structure was heavily biased towards old or very old males who formed coalitions against more recently immigrated young males (Berghänel et al. 2011a; 2011b). In Gibraltar, free-ranging females actively solicited mating from high ranking males during their most likely period of fertility (Brauch et al. 2008), highlighting the important role female behaviour may play for male mating success in this species. 
Barbary macaques are a species with a clear male dominance hierarchy and frequent male coalition formation (Kuester and Paul 1992; Widdig et al. 2000; Bissonnette et al. 2009a; 2009b; 2011; Berghänel et al. 2010; 2011a). It was suggested that frequent triadic male-infant-male interactions (hereafter "male triadic interactions") in Barbary macaques may function to enhance social relationships (Deag and Crook 1971; Paul et al. 1996; Henkel et al. 2010; Berghänel et al. 2011a) and in turn influence coalition formation (Berghänel et al. 2011a). However, Bissonnette et al. (2009a) suggested that coalition formation was opportunistic between males and the highest ranked individual available should always be recruited to maximise the probability of success. Whether males opportunistically select partners for coalitionary aggression or chose partners based on previous experiences was one of the questions I aimed to answer within this thesis.

\subsection{Thesis aims}

The overall aim of my thesis was to examine male-male social relationships, under natural conditions, in a species where females are philopatric, firstly to determine if males formed longterm social bonds. Secondly, I examined if social bond strength provided an adaptive benefit in relation to male-male cooperation. To this end I used a bottom-up approach, by examining the complete chain of events which led to male-male cooperation (Figure 1.1). I determined what males can ascertain about female reproductive state in wild groups of Barbary macaques to gain an empirical estimate of mating contest potential within the group (Chapter 2 ). I then built on this using the PoA model as a base to examine male mating competition and the factors which led to deviation from the PoA models predictions (Chapter 3 ). These species specific measures of male contest provided a further platform for a critical evaluation of the PvS coalition model. Specifically, I examined how the model's predictions fit to empirical data across two species of macaque with variable contest potentials (Chapter 4). Finally, male social relationships were analysed to determine if Barbary macaque males form long-term social relationships, even during intense periods of competition (mating seasons). The strength of male social bonds was examined to see if they provided an adaptive value in terms of cooperation through coalition formation (Chapter 5).

More specifically in Chapter 2, I focused on male mating behaviour and female reproductive physiology, providing the first endocrinological analysis of wild female reproductive physiology and related male mating behaviour. Here I aimed to examine the information males can infer about female reproductive state in order to understand the intensity of male competition and the level of contest potential for the species. I examined female reproductive state across two groups and three mating seasons using a two-fold approach. Firstly, visual assessment of the size 
of the female anogenital swelling was recorded, which reaches maximum size around the time of ovulation. Secondly, I used hormone analysis to examine progestagen metabolites and determine when ovulation most likely occurred. A total 155 samples from 12 cycles of 10 different females were analysed. I then examined if males were able to determine when to concentrate their mating activity around the period of fertility of the female and if they used visual or behavioural cues to determine when to mate. Additionally, I examined male mating behaviour around post-conception swelling periods during gestation to determine if males could differentiate between these and swelling periods when conception could occur. Previous research had compared the size (Möhle et al. 2005) and female vocal calls (Pfefferle et al. 2011) during conception and post-conception periods but no study had compared male mating behaviour during these two periods, which I examined in this thesis.

In Chapter 3, I built on the results provided in Chapter 2 by examining male mating success. More precisely, I looked at whether high ranking males were able to gain as large proportion of matings for their rank position as predicted by the PoA model or which other factors may have reduced the alpha males' ability to monopolise mating access. In this study I added work of Bissonnette et al. (2011) on mating skew in free-ranging Barbary macaques. I examined wild Barbary macaques across three consecutive mating seasons where group composition and age structure was more natural. I used empirical estimates of the information males can infer about female reproductive state to predict male mating skew using the PoA model. In contrast to the previous study, I aimed at teasing apart the factors determining male mating success in a multivariate analysis simultaneously considering male coalitionary activity, female initiated sexual behaviours, as well as the effects of rank and synchrony. 


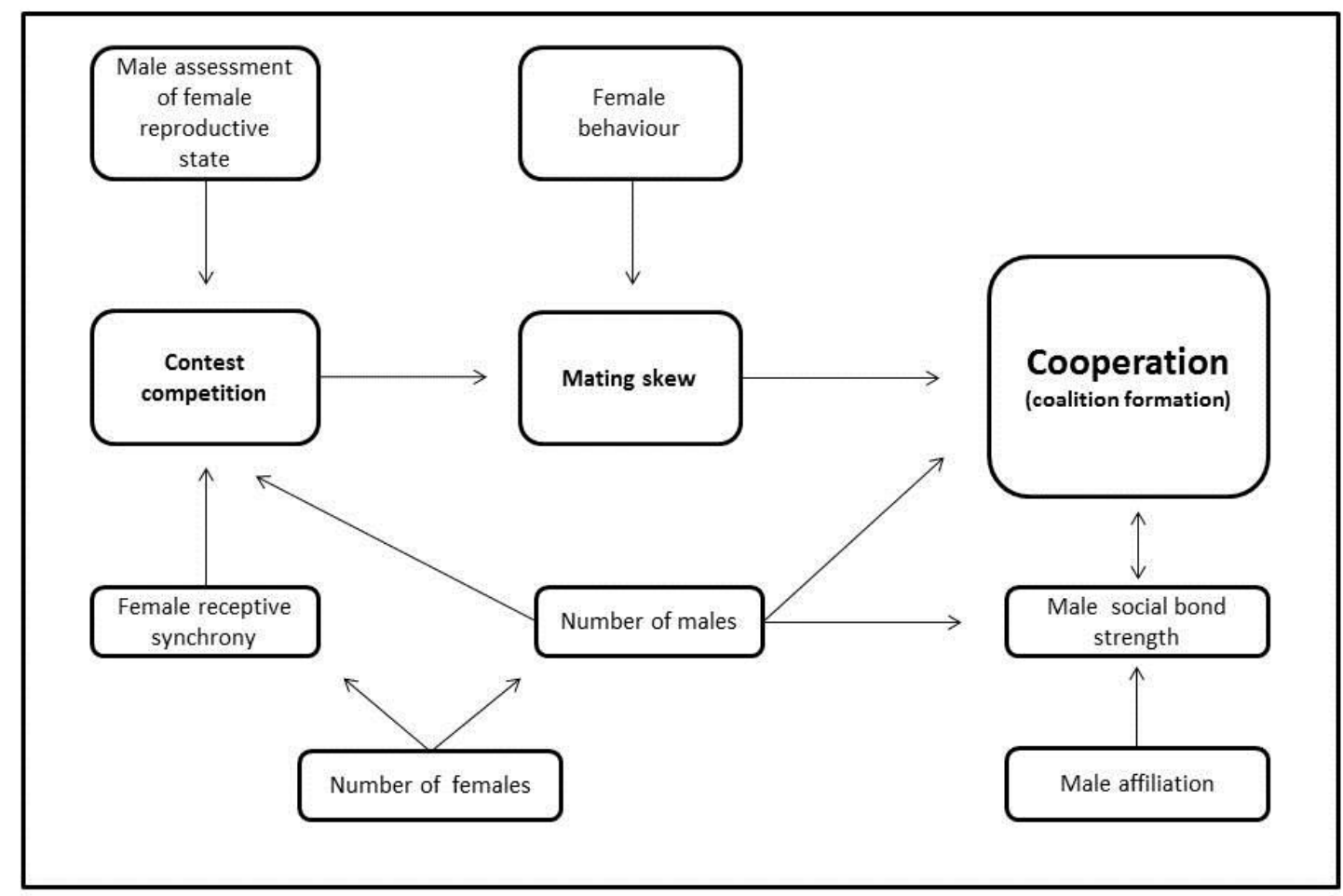

Figure 1.1: Relationships between sexual strategies, male affiliation and coalition formation examined in the thesis. Starting with firstly understanding what males can infer about female reproductive state, before examining male contest competition and mating skew. Finally looking at how male affiliation leads to social bonding between males and ultimately how this all ties into male cooperation through coalition formation.

In Chapter 4, I examined the PvS model for within group coalition formation. The PvS model's predictions have only previously been studied in a handful of species. These studies found support for the model but each was limited by only examining coalitions which occurred at only one specific contest potential. I investigated the occurrence of coalition constellations and types within and across groups of Barbary macaques and across species by including data on male Assamese macaques. By utilizing data on these closely related species I was able to gain contrasting levels of contest potential. This allowed me to fully examine the PvS model across all five coalition types predicted by the model. In particular, I examined how the predictions of the PvS model compared to the actual observed constellations and more specifically, examined the rank of the target and allies of the coalitions as well as the size and contest potential with which each coalition type occurred. 
In Chapter 5, I examined male social bonds and recruitment for coalition formation. The Barbary macaque provided an ideal species with which to examine the strength of social bonds between non-natal cohorts and partner choice during coalitionary recruitment due to the affiliative social style of males, involving grooming, tolerance in social proximity and male triadic interactions combined with abundant male-male coalitionary aggression. I determined male social relationships over an extended time period (over two years including 3 mating seasons) encompassing periods of intense competition between males to understand if social bonds can be sustained throughout these periods and beyond. I then analysed coalition recruitment behaviour to understand if males recruited partners in coalitions due to rank position as suggested by Bissonnette et al. (2011) or due to social bond strength as suggested by Berghänel et al. (2011a). This study advanced to the current literature where coalition frequency has been shown to be related to social bond strength (Watts 2002; Mitani 2006b; Schülke et al. 2010) by examining partner choice in coalitionary recruitment from the available bystanders and relating this to both social bond strength and hierarchal positions of the males involved. 
Chapter 1 


\section{Chapter 2}

\section{Male mating behaviour in relation to female sexual swellings, socio-sexual behaviour and hormonal changes in wild Barbary macaques}

Christopher Young ${ }^{1}$, Bonaventura Majolo ${ }^{2}$, Michael Heistermann ${ }^{3}$, Oliver Schülke $^{4}$ \& Julia Ostner ${ }^{1}$

${ }^{1}$ Primate Social Evolution Group, Courant Research Centre Evolution of Social Behaviour, GeorgAugust University Göttingen, Kellnerweg 6, 37077, Germany.

${ }^{2}$ School of Psychology, University of Lincoln, Brayford Pool, Lincoln, LN6 7TS, U.K.

${ }^{3}$ Reproductive Biology Unit, German Primate Center, Göttingen, Kellnerweg 4, 37077, Germany.

${ }^{4}$ Courant Research Centre Evolution of Social Behaviour, Georg-August University Göttingen, Kellnerweg 6, 37077, Germany.

Hormones and Behaviour 63:32-39 (2013) 


\section{Abstract}

In many cercopithecine primates females display probabilistic cues of fertility to indicate the periovulatory period to males. These cues may include female behaviour, acoustic signals, and morphological signs such as the anogenital swelling. However, the extent to which males can utilise this information varies between species. We describe male sexual behaviour in relation to changes in anogenital swelling size, timing of ovulation and female socio-sexual behaviour in wild Barbary macaques (Macaca sylvanus). We further compare male sexual behaviour during conception and post-conception cycles to evaluate if males differentiate between these qualitatively different cycle types. Our results show that during conception cycles male mating behaviour was concentrated around the fertile phase implying that males inferred information from more than swelling size alone. Male mating frequency increased in line with female sociosexual behaviour, namely female presenting and the frequency of copulations with copulation calls. Most strikingly our results show that males invested equally in mating during fertile and non-fertile, i.e. post-conception, maximum swelling phases. Whether post-conception swellings were merely a result of changes in hormone concentrations during pregnancy or part of a female reproductive strategy remains elusive. In sum, this study adds to the body of research on the evolution of female sexual signals and how males may infer information from these cues.

Keywords

Barbary macaque; Macaca sylvanus; post-conception mating; male reproductive strategies; sexual swellings; progestogens; paternity confusion. 


\section{Introduction}

In most mammalian species females are only sexually receptive around the time of ovulation, the period when conception is possible (McCarthy and Becker 2002). Females may indicate this period by behavioural, visual, olfactory, vocal or tactile cues to males (Lisk et al. 1983; Sherman 1989; Westlin 1996; Rasmussen and Schulte 1998; Swaisgood et al. 2002). Many female primates differ from this general mammalian trend by having a prolonged period of receptivity which extends beyond the fertile phase (Bielert and Anderson 1985; Aujard et al. 1998; Deschner et al. 2004; Engelhardt et al. 2004; Carnegie et al. 2005; for review see Hrdy and Whitten 1987) and even into gestation (Ostner et al. 2006; Engelhardt et al. 2007; Fürtbauer et al. 2011a; Lu et al. 2012). It has been proposed that females use this extended receptivity to both confuse and concentrate mating activity in order to reduce the future risk of infanticide by males (van Schaik et al. 2000). In this study, we investigate male sexual behaviour in relation to changes in female anogenital swelling size, timing of ovulation and female socio-sexual behaviour in Barbary macaques (Macaca sylvanus), a species with prominent sexual swellings.

Anogenital sexual swellings may function as part of a female strategy to balance paternity confusion and concentration in primates living in multi-male, multi-female groups (van Schaik et al. 2000). Sexual swellings may act as probabilistic visual cues to males of the timing of ovulation, reaching maximum size around ovulation (Reichert et al. 2002; Deschner et al. 2003; Brauch et al. 2007; Higham et al. 2008b; for review see Dixson, 1998; Nunn, 1999). By signalling to males the most likely time of ovulation, females enable dominant males to concentrate their efforts around this most important period, while still confusing paternity and mating with multiple males at smaller swelling stages when ovulation is less likely but still possible (Nunn et al. 2001). Across several anthropoid taxa such as baboons (Higham et al. 2009), mandrills (Setchell et al. 2005), macaques (Engelhardt et al. 2004; O'Neill et al. 2004; Higham et al. 2012), and apes (Deschner et al. 2003; Emery and Whitten 2003; Barelli et al. 2008) ovulation occurs during maximum swelling (but see Shelmidine et al. 2007). However, whether males hone further information from more subtle changes in swelling size (Deschner et al. 2004; Higham et al. 2009), olfactory cues around ovulation (Cerda-Molina et al. 2006) or female proceptive behaviour (Wallen et al. 1984; Aujard et al. 1998; Zehr et al. 1998; 2000; Engelhardt et al. 2005) to infer when to concentrate mating varies between species.

In macaques, female proceptive behaviour has been shown to vary during the reproductive cycle and may thus be used by males as a cue to discern the fertile phase. Several studies have shown that the expression of female proceptive behaviours, such as approaching, soliciting and presenting to males is strongly related to changes in oestrogen levels and increase in frequency 
around the fertile phase (Wallen et al. 1984; Aujard et al. 1998; Zehr et al. 1998; 2000). Further research has shown that female sexual behaviour and motivation may be influenced by the social environment; in rhesus macaques, M. mulatta, for example, social group composition (differing male and female composition) influenced how closely related copulation rates were to female reproductive state (Wallen 2001). The social and environmental conditions of primates can therefore influence sexual behaviour so research under natural conditions can build on previous studies under more a controlled environment.

Mating activity in numerous primate species continues into gestation and a few species even exhibit additional sexual swellings during this post-conception, i.e. non-fertile, period, which may serve as an additional mechanism of females to further confuse paternity and decrease the risk of infanticide (van Schaik et al. 2000; 2004b). One of these species is the Barbary macaque, where females show exaggerated swellings both during fertile as well as post-conception phases (Kuester and Paul 1984; Möhle et al. 2005; Brauch et al. 2007). Both swelling types follow similar underlying endocrine changes, particularly an increase in the oestrogen to progestogen ratio (E/P ratio) coinciding with the occurrence of the maximum swelling (Möhle et al. 2005). To date few studies have looked into the function of post-conception swellings and male sexual behaviour in relation to these additional swelling phases in this or other species (Gordon et al. 1991; Gust 1994; Engelhardt et al. 2007).

The aim of this study was two-fold. We firstly aimed to investigate how male Barbary macaques change their sexual behaviours in relation to females' timing of ovulation, changes in swelling size and socio-sexual behaviour. Secondly, we examined whether males differentiate between qualitatively different swelling types by comparing the frequency of male behaviour during conception and post-conception swelling phases. In order to provide the context for these analyses we describe how female sexual swelling size is related to her hormonally assessed fertile phase. 


\section{Methods}

\section{Study site and subjects}

Data were collected from two wild, unprovisioned groups ("Green" (Gn) and "Scarlet" (Sc)) of Barbary macaques living in a deciduous cedar and oak forest in the Middle Atlas Mountains of Morocco $\left(33^{\circ} 24^{\prime} 9 \mathrm{~N}-005^{\circ} 12^{\prime} 9 \mathrm{~W}\right)$. The groups consisted of 7-9 adult males and 8 adult females $(\mathrm{Gn})$ and 6 males and 8 females (Sc), respectively. Data were collected on the Green group from Oct. 2009 - Apr. 2011, data here represent the mating seasons Oct. - Dec. 2009 (Gn09) and Sept. - Dec. 2010 (Gn10). Data on the Scarlet group were collected from Jul. 2010 - Apr. 2011, data here represent the mating season Sept. - Dec. 2010 (Sc10). The mating season was defined as the first to last observed ejaculatory copulation during either focal or ad libitum data collection. This study adhered to the legal requirements of Morocco, Germany and Great Britain.

\section{Behavioural data collection and construction of the hierarchy}

Behavioural data were collected by $\mathrm{CY}$ and 5 field assistants from $0700-1900 \mathrm{~h}$. All adult males in both groups were subject to continuous focal animal observation of social and sexual behaviour (Altmann 1974), yielding a total of 820 focal hours during the mating seasons ( $341 \mathrm{hrs}$ Gn09, 304hrs Gn10 and 175hrs Sc10). Data were collected using handheld HP iPAQ 114 series pocket PCs loaded with Pendragon Forms Version 5.1 (C) Pendragon Software Cooperation, U.S.A.). Focal sampling was randomised within groups and one 40-min focal session per male was recorded per observation day so as to give an even number of focal sessions per individual.

Ejaculatory copulations were recorded and indicated by the occurrence of an ejaculatory pause in pelvic thrusts (Kuester and Paul 1984) and/or the presence of fresh ejaculate around the female's genital area after the copulation. Male inspection (tactile, olfactory or visual) of female's anogenital region as well as female "presenting" behaviour (female presents anogenital region to male; "presenting", Brauch et al. 2007), was also recorded. During ejaculatory copulations the following female socio-sexual behaviours were recorded: the female reaches back to the male during copulation ("reach back", Deag 1974), female looks back at the male during copulation ("look back"), and female vocalizations during copulation ("copulation call", Semple 1998).

Agonistic interactions or conflicts were defined by the occurrence of aggressive (bite, chase, slap, grab, stare, open-mouth, head-bob and charge) and/or submissive behaviours (giveground, make-room, flee, crouch submission). In total 1,238 (Gn) and 195 (Sc) male-male dyadic conflicts were observed where a clear winner and loser of a conflict could be determined with 
no counter-aggression. Based on this, a winner-loser matrix was constructed and a male's dominance rank was assessed using corrected Normalized David's Scores (de Vries et al. 2006) to give a continuous measure of dominance. We used the "Steepness" package (Leiva and de Vries 2011) in R 2.14.0 ( $R$ Development Core Team 2011) based on a matrix of proportions of wins calculated for each dyad.

Faecal sample collection, hormone analysis and definition of the fertile phase

Faecal samples of all adult females of the study groups were collected within 15 minutes of defecation to assess timing of ovulation ( $N=1,066$ samples). Samples were collected for each female when the anogenital swelling began to increase in size towards maximum swelling, throughout the maximum swelling period and 10 days after detumescence. Samples were homogenized and a 3-5g piece was placed in a polypropylene vial, which was in-turn placed in an ice bag and kept cold until samples were transferred to a freezer at $-20^{\circ} \mathrm{C}$ at the end of the day. Samples were transferred in a frozen state to the German Primate Centre for hormonal analysis. Samples were freeze-dried, pulverized and an aliquot of 0.05-0.08 g was extracted with $3 \mathrm{ml} 80 \%$ methanol in water according to the method reported by Heistermann et al. (1995). Extracts were analysed for concentrations of immunoreactive progestagen metabolites ( $5 \alpha$-reduced-20-oxo pregnanes, 5-P-3OH) using a previously validated enzyme immunoassay (EIA) (Möhle et al. 2005; Brauch et al. 2007; Heistermann et al. 2008) and according to procedures previously described by Hodges et al. (1997). Intra- and inter-assay coefficients of variation, calculated from replicate determinations of high- and low-value quality controls, were $5.8 \%$ and $11.5 \%$ (high) and $8.8 \%$ and $13.9 \%$ (low), respectively.

For the two groups and three mating seasons we were able to analyse 23 cycles. However, analysis of behavioural data was restricted to those cycles in which the frequency of faecal sample collection was sufficiently high (sample gap of no greater than 3 days before postovulatory progestagen rise) to allow the estimation of the day of ovulation to an acceptable level of precision (see Heistermann et al. 2008; Dubuc et al. 2011). This gave a total 155 samples from 12 cycles from 10 different females for analysis. The fertile period was determined for each cycle, as previously described by Heistermann et al. (2008). Specifically, a defined rise in faecal 5$\mathrm{P}-3 \mathrm{OH}$ levels above a threshold value (2 SDs above the preceding mean baseline level) was used to determine the most likely day of ovulation (Day 0). As in Brauch et al. (2007), we determined a two day window of the most likely days of ovulation, days -2 to -3 , due to the excretion time lag of faecal progesterone metabolites of 24-56 hours for macaque species (Shideler et al. 1993). The fertile phase consisted of days -2 to -6 relative to the defined faecal 5-P-3OH rise, the 2 day ovulation window plus the preceding 3 days to account for sperm life span in the female 
reproductive tract (Behboodi et al. 1991; Wilcox et al. 1995). For each cycle, the 5 days before the fertile period were defined as the "pre-fertile" period and after as the "post-fertile" period.

\section{Classification of fertile and swelling periods}

Anogenital swelling size was assessed visually using the following approach. In the field swelling size was graded using a three point scale and adapted from the methods of Aujard et al. (1998) and Heistermann et al. (1996), with grade 1 indicating a female with no or minimal swelling or minimal size, grade 2 was given where a partial swelling was present and grade 3 was observed where a maximum swelling was present (tumescence, i.e. the swelling was at maximum size with no wrinkles, and there was protrusion of all genital structures, and this was the period of maximum turgidity).

The maximum swelling period lasted from the first to last consecutive days of a grade 3 swelling (the "maximum swelling" period). We used a combination of hormonal data and counting back from day of parturition $170 \pm 3.77$ days (gestation length: mean \pm SD, Young et al. unpubl. data) from the date of infant birth to estimate if the swelling period corresponded to a conception, pre-conception or post-conception cycle for each female where birth dates were available (20 of 22 females gave birth the following birth season).

\section{Probability of fertility}

We estimated the probability of ovulation for each day of the maximum swelling period $(T)$ by dividing the number of observed ovulations on each day of the maximum swelling by the maximum number of ovulatory cycles examined, following the methods of Deschner et al. (2003). We used the following formula:

$$
P(T=t)=\frac{n_{t}}{n}, t=1,2,3 \ldots
$$

where $t$ is the specific day within the maximum swelling period, $n_{t}$ is the number of cycles in which ovulation occurred on day $\mathrm{t}$, and $\mathrm{n}$ is the overall number of ovulatory cycles. The 5 day fertile phase was determined as above.

To calculate a day specific probability of fertility we took the fertile phase for each cycle and assumed that the probability of fertilisation remains the same for all 5 days. For day of the fertile phase a probability of fertilisation of 1 was assigned and days outside of this period were 
assigned 0 . On any given day of the maximum swelling period ( $f$ ) the average likelihood of fertility was estimated, we summed the scores of probability of ovulation for day $f$ and the following 3 days. This probability is given by:

$$
\mathrm{P}(\mathrm{X}(f)=1)=\sum_{t=f}^{f+3} \mathrm{P}(T=t)
$$

where $\{X(f)=1\}$ is the event of a fertile day and $P(T=t)$ from above.

\section{Statistical analysis}

We compared the maximum swelling period to the fertile phase for the 12 females for whom we had accurate hormonal data. We used a binomial test to examine how many maximum swelling days were also days of the fertile phase. To investigate temporal changes in male sexual behaviour we looked at behaviour around the fertile phase examining mean behavioural rates for the post-fertile phase, the fertile phase and both days -5 to -9 and days -10 to -14 with respect to the fertile phase (days -4 to 0 ). We compared the mean hourly ejaculatory copulation and inspection rates for each female during the pre-fertile, post-fertile and fertile periods using a Wilcoxon signed rank test. Female behaviours are either (copulation call, "look back" and "reach back") expressed as frequency of behaviours females expressed on a specific day relative to the number of copulations females engaged in that day, or (presenting) as the hourly rate per day. Temporal changes in female behaviours were compared using the same methods as for male sexual behaviours above.

Using a Generalized Linear Mixed Model (GLMM; Baayen 2008) (model 1) we tested whether copulation rate was better predicted by days of the maximum swelling period or days of the fertile phase. Copulation rate was measured on the basis of the number of observed ejaculatory copulations per day and included as the dependent variable. Predictor variables were swelling and fertile phase day (was a given day of the mating season a day of maximum swelling or fertile phase period, respectively, 1/0). Fertile phase/maximum swelling day was set to 1 if the corresponding day was within the 5 day fertile or the maximum swelling period respectively and to 0 otherwise. Year, group and female ID were included as random effects and we controlled for effort (log-transformed) as an offset variable in the model (focal hours per day). 
A second GLMM model (model 2) was used to examine how daily male copulation frequency was related to female sexual behaviours and different swelling stages. Copulation rate was measured on the basis of the number of observed ejaculatory copulations per day across all males in the group and included as the dependent variable. The first predictor variable was swelling stage (categorical, 3 levels). Level 1: Non-maximum swelling (period 10 days before and after a maximum swelling), level 2: Conception maximum swelling (period of maximum swelling during a conception cycle), level 3: Post-conception maximum swelling (period of maximum swelling during a post-conception cycle). We also included as continuous predictor variables: the percentage of copulations including female copulation calls, reach backs or look backs as well as the hourly rate of female presenting on a given day. Year, group and female ID were included as random effects and we controlled for effort (log-transformed) as an offset variable in the model (focal hours per day). As the categorical variable swelling stage had three levels we reordered the variable using the "relevel" function so that "conception swelling period" became the reference variable and therefore all levels could be compared to each other.

We ran the GLMM's in R 2.14.0 (R Development Core Team 2011) using the function Imer of the R package Ime4 (Bates and Maechler 2010). All GLMM's were fitted with Poisson error structure and log-link function and likelihood ratio tests were calculated using the $\mathrm{R}$ function anova. Significance of the individual fixed effects was determined based on the $X^{2}$ - and $p$-values provided by Imer. Assumptions for lack of over dispersion and multicollinearity were respected for the analyses.

To compare whether the duration of conception and post-conception swelling periods differed we used a paired t-test. To examine the behavioural influence of post-conception swellings, we compared male sexual behaviours between maximum swelling periods of conception and postconception swellings of ten females. Male sexual behaviour (ejaculatory copulations and inspection rates) and female presenting behaviour, expressed as mean behavioural rates per hour, was compared between the two types of swelling cycles using Wilcoxon signed rank tests.

Where appropriate, we report mean values \pm standard deviation (SD). All statistical analyses were carried out using R 2.14.0 software (R Development Core Team, 2011). The level of significance was set at $\alpha<0.05$. 


\section{Results}

\section{Probability of fertility}

There was a temporal correspondence between the maximum swelling period and the fertile phase for the 12 female cycles. Results indicated that a significant number of days (65\%) of the fertile phase fell within the maximum swelling period (binomial test, $x=39, N=60, p<0.027$; Figure 2.1). The probability of ovulation was highest on days -2 and $-4(0.167)$, relative to the last day of maximum tumescence (day 0), and slightly lower on day -3 (0.125) giving a peak period of ovulation probability on these 3 days (Figure 2.2). The combined probability of ovulation across these 3 days was 0.46; however, ovulation could occur on any day from day -9 to +1 . Consequently, fertilisation was possible from days -13 to +3 and was most probable between days -2 and -6 reaching a peak on day -4 (0.625; Figure 2.2).

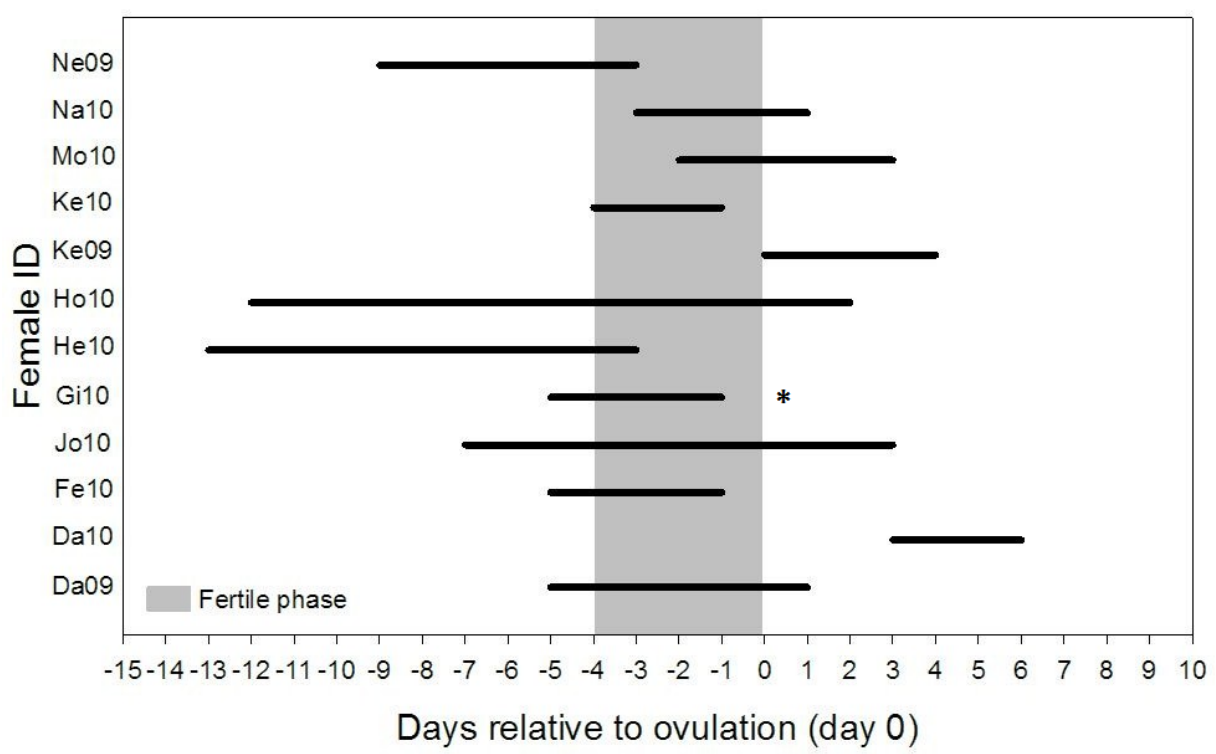

Figure 2.1: Duration and timing of the maximum swelling periods relative to day of ovulation (day 0). Periods of maximum swelling for 10 females and 12 ovulatory cycles are each represented by horizontal lines. The shaded area represents the fertile window. The identity of each female is given along with the mating season year $(09=2009,10=2010)$. Non-conceptive cycles are indicated by an asterisk. 


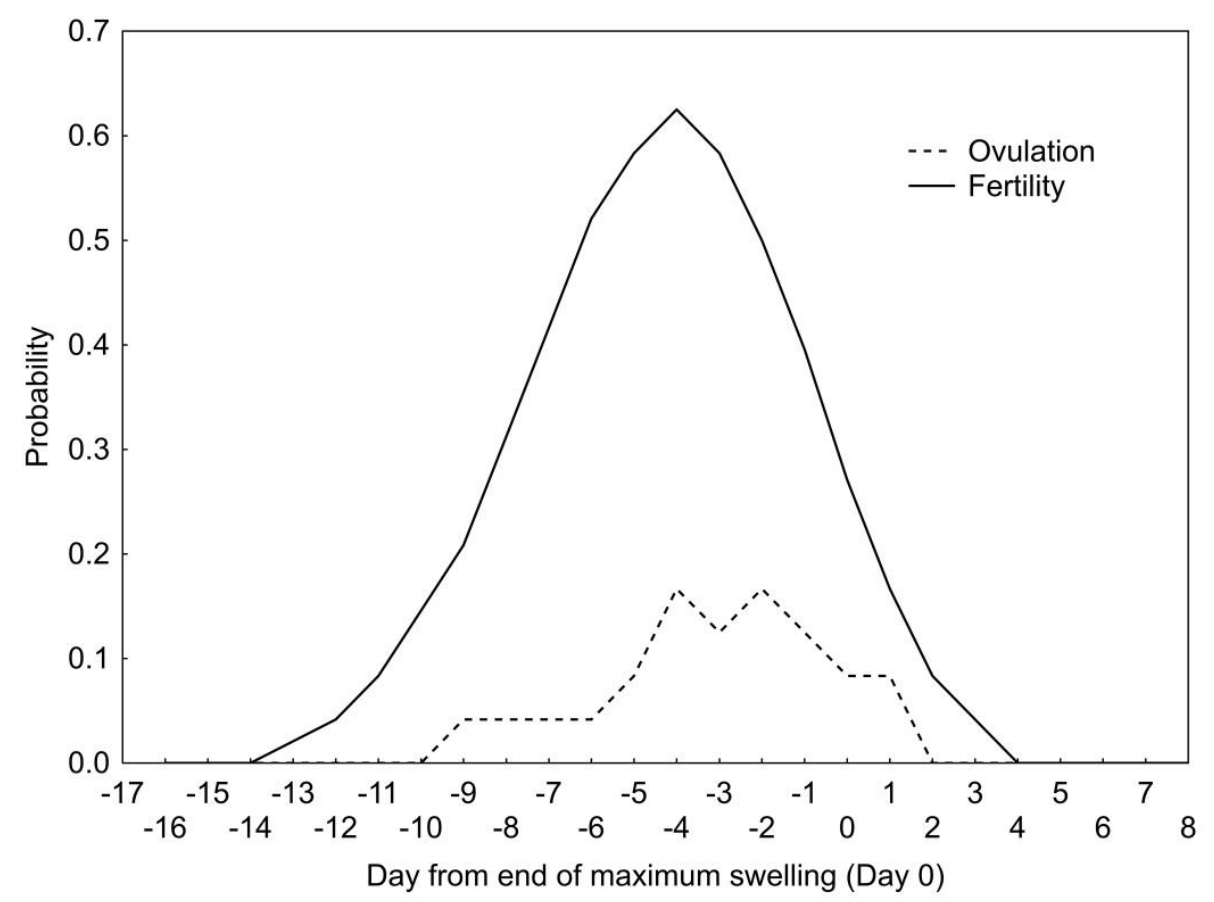

Figure 2.2: Probability of ovulation (dashed line) and fertility (solid line) relative to the last day of maximum tumescence (day 0 ; see methods for details).

Male sexual behaviour around the fertile phase

Mean hourly rates of male sexual behaviour during different periods of female fertility were examined comparing the fertile period with the pre-/post-fertile periods respectively. In separate tests rates of ejaculatory copulations as well as inspection rates were significantly higher in the fertile as well as the pre-fertile period compared to the post-fertile period, while there was no difference between the pre-fertile and the fertile periods (Figure 2.3). Ejaculatory copulation rates: Pre-fertile vs. fertile: $V=63, p=0.064$; fertile vs. post-fertile $V=75, p=0.002$; pre-fertile vs. post-fertile: $V=47, p=0.053$. Inspection rates: Pre-fertile vs. fertile: $V=52, p=$ 0.340 ; fertile vs. post-fertile $V=71, p=0.010$; pre-fertile vs. post-fertile: $V=12, p=0.034, N=12$ for all tests.

A GLMM (model 1) revealed that male mating rate was predicted by days of the fertile period but not by days of the maximum swelling period (Table 2.1). 

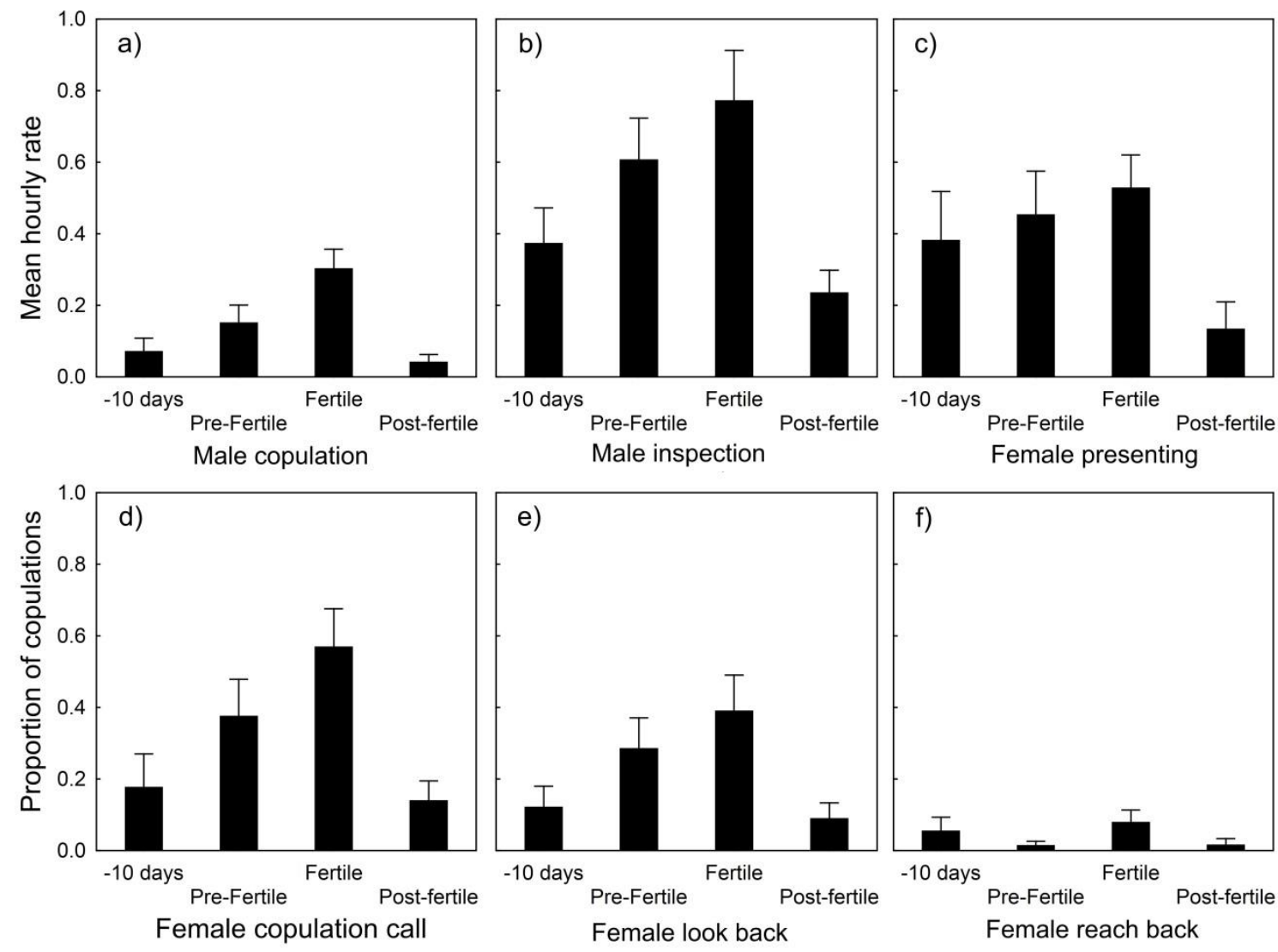

Figure 2.3: Comparison of male and female sexual behaviour during different stages of female fertility concerning (clockwise from top left) a) mean ejaculatory copulation rate b) mean inspection rate c) mean female presentation rate d) proportion of copulations during which females produced copulation calls, e) look-backs, f) reach-backs. Values were averaged across all cycles $(N=12)$ and represent the mean \pm SEM.

\section{Comparing the conception and post-conception maximum swelling periods}

The duration of the conception maximum swelling period (mean $\pm S D=8.27 \pm 2.45$ days) was not significantly different from the maximum swelling post-conception period (mean $\pm S D=6.64$ \pm 2.5 days; $t$-test: $t=1.54, d f=20, p=0.14$ ). Post-conception swellings occurred $25-30$ days after detumescence of the conception swelling period and the vast majority of females $(80 \%)$ developed a post-conception swelling. No more than one post-conception swelling was observed for any female. 
Table 2.1: GLMM Poisson regression results for the relationship between male ejaculatory copulation rate on a given day and whither this is a day of the fertile phase (determined hormonally) or the maximum swelling period $(N=628)$.

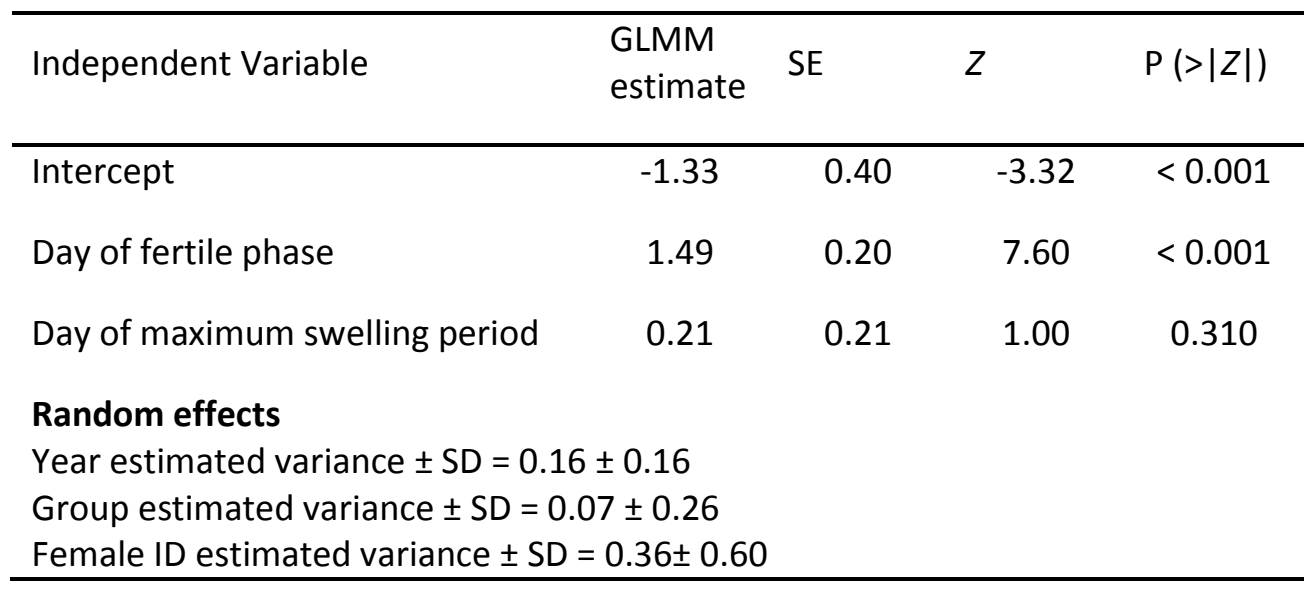

There was no significant difference in mean hourly frequency of male matings or inspections between the conception and post-conception swelling periods (ejaculatory copulation rate: mean \pm SD $=0.231 \pm 0.14$ (conception maximum swelling), $0.235 \pm 0.22$ (post-conception maximum swelling); $V=32, p=0.70$; inspection rate: mean $\pm S D=0.553 \pm 0.22$ (conception maximum swelling), $0.702 \pm 0.51$ (post-conception maximum swelling); $V=16, p=0.28, N=10$ for both tests, Figure 2.4). The alpha males were no exception and did not concentrate their mating efforts to conceptive swelling phases (ejaculatory copulation rate: mean $\pm S D=0.03 \pm$ 0.02 versus $0.05 \pm 0.03$ during conception and post-conception maximum swelling phases, $V=0$, $p=0.37, N=3)$. The mean hourly frequency of female presenting also did not differ significantly between the two swelling periods (mean $\pm S D=0.471 \pm 0.35$ (conception maximum swelling), $0.490 \pm 0.33$ (post-conception maximum swelling); $V=24, p=0.77 . \mathrm{N}=10$ ). 


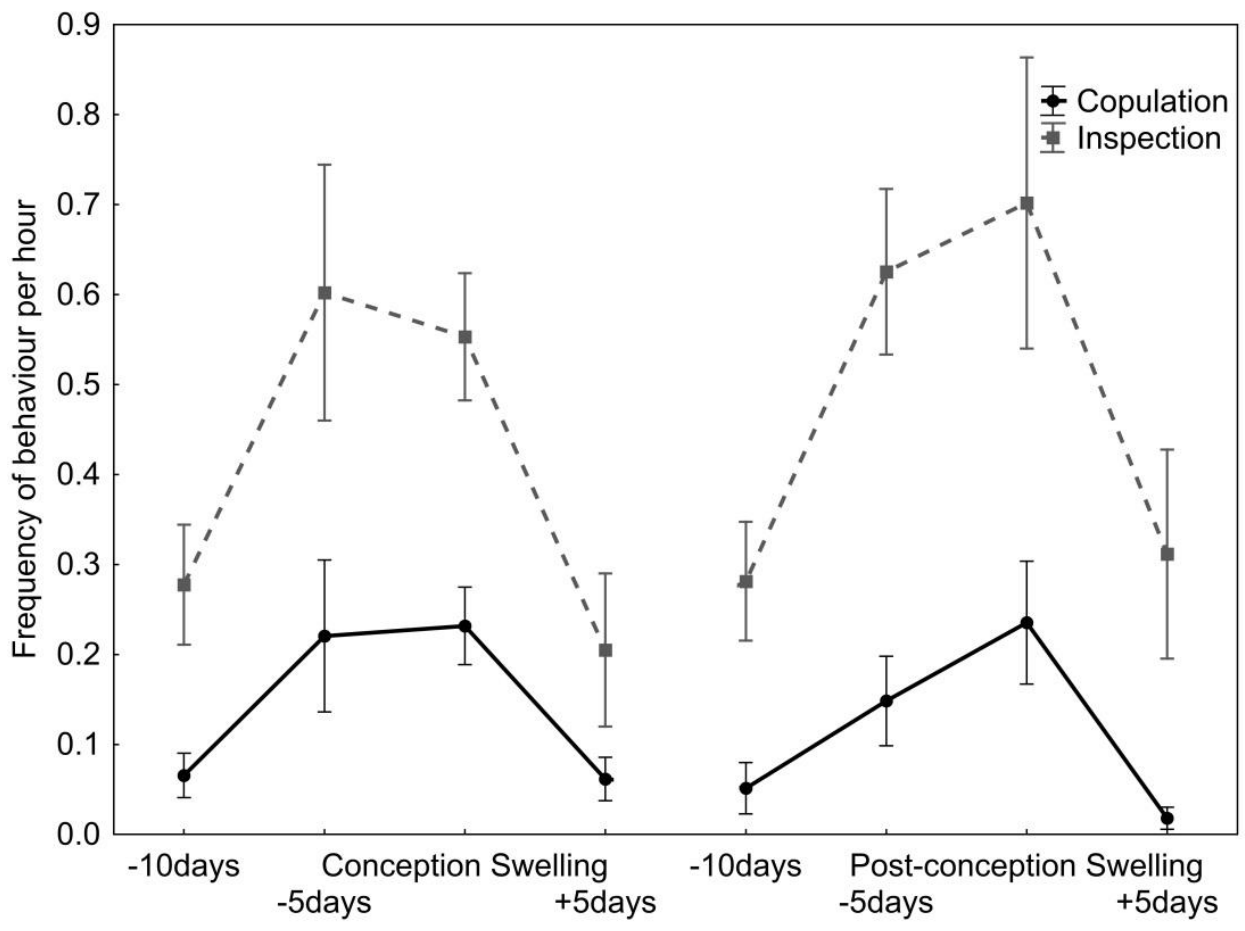

Figure 2.4: Mean hourly rate of male copulations and inspections in relation to the different maximum swelling periods, as well as the 5 days after the swelling period (+5days) and days 1-5 (-5 days) and days 6-10 (-10 days) before the swelling period. Values were averaged across all cycles $(N=10)$ and represent the mean $\pm S E M$.

Male sexual behaviour in relation to female socio-sexual behaviour

In the second GLMM (model 2) we examined how the males' copulation behaviour was related to a given female's swelling state, her conception status, and her socio-sexual behaviour on a given day. The copulations all group males engaged in were more frequent on days of maximum swelling size, no matter whether the female was fertile or already pregnant. The males also copulated more the more frequently a female presented and the higher the proportion of her copulations that were associated with copulation calls. The proportion of copulations that were associated with the more subtle female behaviours of looking back at the copulating male or reaching back to him were not related to the males' copulation rate (Table 2.2). 
Table 2.2: GLMM Poisson regression results for the relationship between male ejaculatory copulation rate on a given day and female sexual behaviours (female presentations per hour, percentage of copulations with copulation calls, look backs and reach backs) and different maximum swelling periods (conception maximum swelling, post-conception maximum swelling or the 10 days before and after a swelling period) $(N=415)$.

\begin{tabular}{|c|c|c|c|c|}
\hline Independent Variable & $\begin{array}{l}\text { GLMM } \\
\text { estimate }\end{array}$ & SE & $z$ & $P(>|Z|)$ \\
\hline Intercept & -3.35 & 0.31 & -10.70 & $<0.001$ \\
\hline $\begin{array}{l}\text { Conception max-swelling Vs. Post- } \\
\text { conception max-swelling }\end{array}$ & -0.24 & 0.18 & -1.33 & 0.184 \\
\hline $\begin{array}{l}\text { Conception max-swelling Vs. Non- } \\
\text { max-swelling }\end{array}$ & 0.61 & 0.16 & 3.91 & $<0.001$ \\
\hline $\begin{array}{l}\text { Post-conception max-swelling Vs. } \\
\text { Non-max-swelling }\end{array}$ & 0.36 & 0.17 & 2.21 & 0.030 \\
\hline Female presentations $/ \mathrm{hr}$ & 0.52 & 0.08 & 6.77 & $<0.001$ \\
\hline$\%$ copulations with copulation call & 2.29 & 0.26 & 8.88 & $<0.001$ \\
\hline$\%$ copulations with look back & 0.16 & 0.20 & 0.82 & 0.410 \\
\hline$\%$ copulations with reach back & -0.22 & 0.24 & -0.93 & 0.350 \\
\hline $\begin{array}{l}\text { Random effects } \\
\text { Year estimated variance } \pm S D=0.00 \\
\text { Group estimated variance } \pm S D=0 . \\
\text { Female ID estimated variance } \pm S D\end{array}$ & $\begin{array}{l} \pm 0.00 \\
0 \pm 0.00 \\
0.61 \pm 0.78\end{array}$ & & & \\
\hline
\end{tabular}

\section{Discussion}

Combining hormonal measurements with visual scoring of swelling sizes we found swellings to be a probabilistic indicator of fertility as the occurrence of ovulation was most likely during the maximum swelling period but the timing of ovulation within the swelling varied. Male copulation frequency was higher during maximum swelling periods than outside this period but mating rate was still better predicted by the hormonally assessed fertility than by swelling size, suggesting that more than the maximum swelling size alone was used by males to determine female attractivity. Male mating behaviour was additionally predicted by female socio-sexual behaviour, namely female presenting and the percentage of copulations with copulation calls, however, whether males use these behaviours or additional information as cues to allocate their mating 
activity was beyond the scope of this study. Most strikingly, males engaged at equal rates in sexual behaviour during fertile and post-conception swelling stages, despite the fact that the mating during the latter by definition cannot lead to fertilisations and thus only carries costs for the male.

There was a temporal correspondence between maximum swelling size and fertility with the majority of maximum swelling days also being fertile days, i.e. days when ovulation was most likely. The actual day of ovulation did vary, however, within the maximum swelling period as has been shown in several other species (Reichert et al. 2002; Deschner et al. 2003; Higham et al. 2008b) including semi-free ranging Barbary macaques (Möhle et al. 2005). The probability of fertility peaked 5 days before detumescence, which is consistent with chimpanzees (Deschner et al. 2003) and one day less than in baboons (Higham et al. 2008a). In addition to maximum swellings during conception, $80 \%$ of females also had a post-conception maximum swelling period, a finding very similar to that reported for semi-free ranging animals (Möhle et al. 2005). In macaques, maximum swelling size is preceded by endocrine changes with a decrease in progestogen levels and an increase in oestrogen levels and thus a significant rise in the E/P ratio which is similar during maximum swelling for both conception and post-conception periods (Möhle et al. 2005; Engelhardt et al. 2007). If males were to use the absolute swelling size or the underlying endocrine changes as a cue when to concentrate mating then the similarity of swelling periods could cause confusion.

During the conception cycle, males concentrated their mating activity around the fertile period, when fertilisation was most likely, suggesting that males may use further cues than absolute swelling size alone to determine when to mate. Male copulation rates gradually increased towards the fertile period and decreased abruptly afterwards (Figure 3), which is consistent with previous data on semi-free ranging Barbary macaques (Heistermann et al. 2008). Males in several other species also concentrate mating around the fertile period (Wallen et al. 1984; Aujard et al. 1998; Deschner et al. 2004; O'Neill et al. 2004; Engelhardt et al. 2005; Higham et al. 2009; 2012) nevertheless; the exact cues males use to determine the fertile phase varies. Inspection rates greatly decreased in frequency after the fertile phase but were similar in the pre-fertile and fertile phases, although the mean frequency was still greatest during the fertile phase (Figure 3). This implies that males inspected swellings and the anogenital region in order to gain information about the females' reproductive state, similar to a previous study (Heistermann et al. 2008). Close inspection of the female's anogenital region provides a male with further cues to the probability of ovulation such as fine grained visual changes in swellings size or coloration (Deschner et al. 2003; Möhle et al. 2005) or olfactory cues (Cerda-Molina et al. 
2006), to date, however, evidence of olfactory detection in macaques is limited (for review see Dixson, 1998). Our data suggest that males may be able to pick up on further hormonal cues given that the inspection rate increased towards the fertile phase and male mating frequency was better predicted by days of the fertile phase than days of the maximum swelling period. But the precise cues males use to infer this additional information could not be disentangled by this study.

The frequency of female socio-sexual behaviours is known to be related to the concentration of oestrogens; in rhesus macaques' oestrogen rise as ovulation approaches coincides with a rise in frequency of female proceptive behaviours such as approaching, soliciting and presenting (Wallen et al. 1984; Zehr et al. 2000). Similarly, Tonkean macaque ( $M$. tonkeana) females increase the frequency of presenting (Aujard et al. 1998) and long-tailed macaques ( $M$. fascicularis) show more reach back behaviour around the fertile phase (Engelhardt et al. 2005), suggesting that female socio-sexual behaviours parallel the changes in female reproductive state. The increase in frequency of these behaviours may be a cue used by males to determine when to concentrate mating effort and in all three macaque species male mating behaviour reaches its peak around the fertile phase. Female socio-sexual behaviour, specifically presenting and copulation call frequency, in our study increased in parallel to male copulation rate, increasing in frequency as the fertile phase approached, peaking during the fertile phase and decreasing afterwards. Our data are thus consistent with the idea that males respond to changes in female behaviour; although which cues males use exactly remains to be investigated.

While males seemed to use these additional cues to concentrate mating around ovulation, all males including the alpha male mated at the same rate during conception and post-conception swelling phases. In addition, male inspection and female presenting frequencies did not differ between these two swelling phases. Similarly, long-tailed macaque copulation rates and female socio-sexual behaviour also remained similar during conception and post-conception periods (Engelhardt et al. 2007). Mating during gestation is not uncommon in macaque species (Wilson et al. 1982; Nieuwenhuijsen et al. 1986; Engelhardt et al. 2007; Fürtbauer et al. 2011a) and may be linked to distinct endocrine changes during pregnancy. Both a decrease in progestogen levels in association with a parallel increase in oestrogen concentrations (Wilson et al. 1982) and a resultant increase in the E/P ratio (Bielert et al. 1976; Engelhardt et al. 2007) are known to coincide with increased mating frequencies during gestation. As the increase in size of the swelling and change in E/P ratio occur during both conception and post-conception swelling periods it is difficult to tease apart which, if any, cue males use for deciding if and when to mate. Whether these additional swellings have an adaptive function or not is uncertain (reviewed by 
Engelhardt et al. 2007) but indirectly they are costly to males, who invest time and energy in vain. Post-conception swellings can enhance promiscuity by adding another mating cycle to the reproductive phase of a female.

Across primates female reproductive synchrony is the main determinant of male monopolization potential and consequently reproductive skew (Ostner et al. 2008b). By producing a postconception swelling female Barbary macaques in this study increased reproductive synchrony by $25 \%$ (from $32 \%$ overlap (excluding post-conception cycles) to $40 \%$, Young et al. unpubl. data) effectively reducing male monopolisation potential and possibly enhancing paternity confusion. Whether post-conception swellings are a further development in the inter-sexual arms race between males and females to maximise female reproductive potential and limit the information available to males (van Schaik et al. 2004b) or a purely by-product of changes in hormonal concentrations needs further studies.

Taken together, our study provides new insights into the information available to males, namely female socio-sexual behaviour, hormonal cues and sexual swellings and how males "respond" to them in terms of allocating their copulatory activity. Perhaps most interestingly, our results raise intriguing questions about the underlying mechanisms and function of post-conception swellings, which were beyond the scope of this study. Future studies examining mating behaviour during gestation swelling periods form both a male and female perspective could shed light on a possible adaptive or mechanistic function of these swellings. Females with additional conspicuous swellings could endure costs in terms of energy expenditure during pregnancy and increased behavioural and time constraints imposed by greater attractiveness to males. Detailed evaluation of changes in female hormone concentrations, in particular the E/P ratio and finegrade changes in swelling size during post-conception swelling periods may enhance our understanding of the underlying mechanisms of these swelling periods. If males do follow finescale changes in swelling size or infer information from changes in hormonal concentrations as to when to mate this would, in multi-male multi-female groups over a mating season, require males to monitor changes of all females over time within the group. Whether, over several months, cercopithecine primate males have the cognitive abilities required to maintain such detailed information would require further research. By considering primate sexual behaviour outside periods surrounding fertility future research into the evolution of female sexual signals may be enhanced. 


\section{Acknowledgments}

The authors are grateful to Professor Mohamed Qarro (Ecole Nationale Forestière d'Ingénieurs, Morocco) for his support in the field and the Haut Commissariat aux Eaux et Forêts et à la Lutte Contre la Désertification of Morocco for research permission. We would also like to thank Michael Madole, Dave Thomas, Sofia Santos, Maria Thunström, Tom Smith and Josephine Msindai for assistance in the field and Ines Fürtbauer and Laëtitia Maréchal for helpful comments. We would also like to thank Andrea Heistermann and Petra Kiesel for providing assistance and expertise during hormone analysis. Furthermore, we would like to thank Dr. Kim Wallen and two anonymous referees for useful comments to significantly improve the manuscript. Financial support was provided by the Max Planck Society, the Christian Vogel Fonds and Georg-August University Göttingen through funds from the German Initiative of Excellence. This study complies with Moroccan, German and UK regulations regarding the ethical treatment of research subjects. 


\section{Chapter 3}

\section{Male coalitions and female behaviour affect male mating success independent of dominance rank and female receptive synchrony in wild Barbary macaques}

Christopher Young ${ }^{1}$, Sabine Hähndel ${ }^{1}$, Bonaventura Majolo ${ }^{2}$, Oliver Schülke ${ }^{3^{*}}$ \& Julia Ostner ${ }^{1^{*}}$

${ }^{1}$ Primate Social Evolution Group, Courant Research Centre Evolution of Social Behaviour, GeorgAugust University Göttingen, Kellnerweg 6, 37077 Germany.

${ }^{2}$ School of Psychology, University of Lincoln, Brayford Pool, Lincoln, LN6 7TS, U.K.

${ }^{3}$ Courant Research Centre Evolution of Social Behaviour, Georg-August University Göttingen, Kellnerweg 6, 37077 Germany.

*equal contribution as last authors 


\section{Abstract}

Dominant mammalian males should gain a reproductive advantage due to their greater fighting abilities. However, the extent to which they can monopolise access to females varies across species. In primates and recently other mammalian species, the Priority of Access (PoA) model is commonly used to measure the degree to which male rank and female receptive synchrony affect mating skew. Few studies have examined the factors which led to deviations from the expectations of the model. Here we investigate male mating skew in wild Barbary macaques (Macaca sylvanus). We examined four of the main factors which affect male mating success: the roles of male rank, female receptive synchrony, coalitionary activity, and female behaviour. We found that male mating was skewed up the hierarchy but there was a large deviation from the PoA model's expectations with high ranked males not gaining as big a share as expected. Females frequently initiated sexual encounters, predominantly with mid-ranked males, increasing their mating success. Male coalitionary activity independently increased mating success. Frequent associations with females were costly to males as they were the targets of bridging coalitions, decreasing future mating opportunities for the targets. High ranking males did not increase their mating success directly through bridging coalitions but acted to dilute the effects of female behaviour. By examining different factors affecting mating skew we are able to show that alternative male and female mating strategies are effective in reducing the monopolisation potential of the dominant male.

Keywords

Priority of Access model; mating skew; coalition; female mate choice; reproductive strategies; Macaca sylvanus. 


\section{Introduction}

Potential reproductive rates are usually higher among mammalian males compared to females, leading to male-biased operational sex ratios and consequently pronounced male-male competition for mating opportunities (Trivers 1972; Clutton-Brock and Parker 1992; Kvarnemo and Ahnesjö 1996). Under these conditions, sexual selection favours traits that give males an advantage in physical contests such as body size, strength, weaponry and endurance (Plavcan and van Schaik 1997; Setchell 2003; Plavcan 2004; Emlen 2008). If males cannot exclude rivals from their group, reproductive competition and access to mating partners is often mediated via dominance rank among co-resident male rivals (Altmann 1962), with dominant males using their superior fighting abilities to defend access to receptive females (Shively and Smith 1985; Bercovitch 1988). How strongly male mating success is predicted by dominance rank, however, varies greatly, within and between species, particularly so among primates (Cowlishaw and Dunbar 1991; van Noordwijk and van Schaik 2004; Kutsukake and Nunn 2006).

The extent to which a dominant male can monopolise mating access to females within a group has been conceptualized in the Priority of Access model (hereafter the PoA model; Altmann 1962), which proposes that the ability of a dominant male to monopolize females depends on the degree of reproductive synchrony among females. The reasoning being that the dominant male can defend only one female at a time, hence if there are two females simultaneously receptive, the second ranking male will also get his share of matings and if there are three females receptive, the third ranking male will also mate, and so on. The importance of reproductive synchrony as a predictor of alpha male mating or reproductive skew has been confirmed in comparative studies across primates (Kutsukake and Nunn 2006; Ostner et al. 2008b; Gogarten and Koenig 2012). The PoA model has often been applied in studies on primates (see Kutsukake and Nunn 2006) and more recently other mammalian species (Hirotani 1994; Say et al. 2001; Engh et al. 2002). For species exhibiting variable female receptivity, the PoA model may be favourable for tests of mating/reproductive skew compared to theoretical reproductive skew models as the former incorporates female receptive synchrony in the model, which is neglected by many reproductive skew models, but can be an important factor to consider in multi-male, multi-female groups (Port and Kappeler 2010). While most studies investigating the relationship between dominance rank and mating or reproductive success found a positive relationship (Cowlishaw and Dunbar 1991; Ellis 1995; Alberts et al. 2003; Majolo et al. 2012), it is also apparent that the strength of the relationship varies extensively across species of different genera, across species of the same genus or even within the same group over time (reviewed in Alberts 2012). So far only a handful of studies directly tested the exact 
PoA expected distribution against the observed skew and investigated the factors driving the departure from the model (Alberts et al. 2003; Boesch et al. 2006; Hayakawa 2008; Wroblewski et al. 2009; Bissonnette et al. 2011; Dubuc et al. 2011)

A number of factors are thought to explain the deviation from the PoA model's expectations or more generally the variation in individual male mating success: energetic costs of mate-guarding, stability of the dominance hierarchy, invasions by non-resident males, male sexual coercion, male coalition formation, and female mate choice (reviewed in Alberts 2012). Extended mateguarding is energetically costly to males as it reduces foraging activity (Packer 1979; Alberts et al. 1996; Coltman et al. 1997; Mainguy and Côté 2008; Pelletier et al. 2009; Schubert et al. 2009) which may reduce mating success of high ranking males (especially if coupled with large female group size and aseasonal reproduction, Alberts et al. 2003). Noë (1992) proposed a modifiedPoA model where only the highest ranking male baboons (Papio sp.) would benefit from extended mate guarding of females and males below top should break up consorts using coalition formation to gain access to females (see below). Seasonal influxes of non-resident males have also been proposed as a way to break up the dominant male's monopoly, however, those invasions have mostly been observed in single male groups possibly because multi-male groups are usually able to repel intruders (Cords 2000; but see Borries 2000; reviewed by Alberts 2012). Instability in the male dominance hierarchy may also reduce the alpha male's ability to monopolise access to receptive females as challenges from rivals for the alpha position are likely to increase during periods of instability (Cowlishaw and Dunbar 1991).

Males in multi-male groups can use aggressive strategies to attempt to increase their mating success with certain females, such as sexual coercion (Muller and Wrangham 2009). Males may use aggression to intimidate a female before her receptive period, "punish" her when she refuses to mate or after mating with other males (Muller et al. 2007; 2011). Alternatively, males can access receptive females through cooperation and combining their intrinsic fighting abilities via coalition formation (Noë and Sluijter 1990; Kuester and Paul 1992). By targeting a higher ranking male and preventing him from mating, allies may effectively re-allocate matings towards subordinate males ("levelling coalitions"; Pandit and van Schaik 2003). In the case that a nondominant male receives a disproportionate share of mating success, one may expect that coalitions will target those males - regardless of their relative dominance rank. As well as a temporal loss of mating opportunities, prolonged coalition formation against a target can also lead to intimidation of the target (van Schaik et al. 2006; Berghänel et al. 2011b) and cause further negative consequences which may ultimately led to reduced future reproductive success. Levelling coalitions can be of the "all-up" type (van Schaik et al. 2004a, also termed 
"conservative" by Chapais 1995), where two low-ranking males team up against a higher ranking target, or "bridging" where a high and a low ranking male target an intermediate individual. Levelling coalitions have been observed in a range of primates, most predominantly in baboons and macaques (Smith et al. 2010), where they can have a pronounced effect on the mating distribution across males (reviewed in van Schaik et al. 2004a). Additionally, "all-down" coalitions (two higher ranked males aggressing a lower ranked target) are predicted to occur whenever all-up and bridging coalitions occur with a defensive function or to cause intimidation (van Schaik et al. 2004a).

Females can also influence the rank-based distribution of mating access, concentrating mating towards one male or mating promiscuously (Dixson 1998; Drea 2005). Female primates need to ensure that all males have a non-zero chance of paternity in order to reduce the risk of infanticide (van Schaik et al. 2004b). Assuming that higher ranked males gain matings via their superior rank positions, females may thus preferentially mate with males at the lower end of the PoA expected mating distribution. Females can engage in certain strategies to attempt to achieve this by, for example, initiating more sexual encounters with particular males (Janson 1984), by mating conspicuously out of sight of other group members ("sneak copulations"; Berard et al. 1994; Alberts et al. 2006; Overduin-de Vries et al. 2012) or by selectively refusing copulations from certain males (Huffman 1987; reviewed by Dixson 1998; Drea 2005). Female primates may conceal ovulation or lengthen receptivity increasing receptive synchrony within groups which, in turn, reduces the ability of the alpha male to monopolise access and increases the opportunity for mate choice (reviewed by Kappeler 2012).

Here we examined the factors determining male mating success in Barbary macaques (Macaca sy/vanus), a species with a clear male dominance hierarchy and frequent male coalition formation (Kuester and Paul 1992; Berghänel et al. 2010; 2011a; Bissonnette et al. 2011). A previous study under free-ranging conditions at Affenberg Salem examined male mating success (Bissonnette et al. 2011) and found a poor fit of the observed mating skew to the PoA model. Low-ranked, post-prime males increased their mating success using coalitionary activity to target high-ranked males breaking up consortships in all-up levelling coalitions. Females initiated consortships with higher ranked prime-males to possibly counteract this coalitionary behaviour. In this provisioned group male and female group size was largely inflated and age structure was heavily biased towards old or very old males who formed coalitions against more recently immigrated young males (Berghänel et al. 2011a; 2011b). In Gibraltar free-ranging females actively solicited mating from high ranking males during their most likely period of fertility (Brauch et al. 2008), highlighting the importance of female behaviour for male mating success in 
this species. In this study we build on the work of Bissonnette et al. (2011) by studying wild Barbary macaques across three consecutive mating seasons living in a group with a more natural composition and age structure. In contrast to the previous study, we aimed at teasing apart the factors determining male mating success in a multivariate analysis simultaneously considering male coalitionary activity, female initiated sexual behaviours, as well as the effects of rank and synchrony.

The effect of female reproductive synchrony can vary between species depending on the information males can infer about female fertility and thus needs to be based on the speciesspecific fertility information available to males (Alberts et al. 2006; Wroblewski et al. 2009; Henzi et al. 2010). In the case of Barbary macaques we have previously shown that males bias their mating behaviour towards periods of maximum sexual swellings, but do not differentiate between swellings during the conceptive phase, determined through hormonal assessment of the timing of ovulation, and those during gestation (Young et al. 2013b). We thus, include both cycle types in our estimate of female reproductive synchrony. We do not investigate the potential effects of male invasions because this should mainly play a role in one male groups (see above) and because they were not observed during the study. Similarly, male sexual coercion occurred at very low levels during the study and thus we only reported the individuals involved in these behaviours. The energetic costs of mate-guarding should not influence mating success in the species as mate-guarding episodes in Barbary macaques are very limited in time (Kuester and Paul 1992; Heistermann et al. 2008; Bissonnette et al. 2011). It could be argued that with high levels of promiscuity and short consortships the assumptions of the PoA model are not upheld in the species. However, male rank may still play a major role, despite a lack of extended consortships, in regulating access to receptive females with increasing female receptive synchrony reducing the alpha male's ability to monopolise mating access. When synchrony is low, subordinate males may shy away from mating opportunities due to intimidation or fear of aggression from higher ranked males and, as more females become synchronously receptive, the opportunities of subordinates to mate will increase. Thus, even in species without extended mate-guarding periods the rationale of the PoA model may still hold with high ranking males losing control of mating access as more females become receptive.

We examined the role of four different factors in predicting male mating success in two steps. In the first step, we derived for each of the three mating seasons a mating distribution in relation to rank as predicted from the PoA model by considering both rank and the number of synchronously receptive females. We tested whether 1 ) observed and predicted mating distributions per season were significantly skewed, 2) mating success was correlated to rank, 3) 
the lowest rank that received any mating equalled the maximum number of synchronous females, and 4) qualitatively assessed whether observed and expected mating distributions were similar. In a second step, we analysed daily male mating success and how it related to male rank position, female receptive synchrony, and their interaction, as well as, male coalitionary activity and the frequency of female initiated sexual encounters. We expected males to manipulate the mating distribution via coalitions that prevented other males from access to females. If females were influencing males' mating success we expected males with a greater frequency of sexual encounters initiated by females to have an increased mating success. We also expected those males who received the greatest frequency of sexual encounters initiated by females to become the most frequent targets of coalitionary aggression as other group males attempted to disrupt their mating opportunities.

\section{Methods}

\section{Study site and subjects}

The study was conducted on one wild, unprovisioned group of fully habituated Barbary macaques living in a deciduous cedar and oak forest in the Middle-Atlas Mountains of Morocco $\left(33^{\circ} 24^{\prime} 9 \mathrm{~N}-005^{\circ} 12^{\prime} 9 \mathrm{~W}\right)$ (Majolo et al. 2013). The study population breeds seasonally with a mating season from Sept. to Dec. and a corresponding birth season from Mar. to May. Data for this study were collected during three consecutive mating seasons (MS09: Oct.-Dec. 2009, MS10: Sept.-Dec. 2010, MS11: Sept.-Dec. 2011). The mating season was defined as the period of the first to the last observed ejaculatory copulation during either focal or ad libitum data collection. During the study period, the study group ("Green" group) consisted of 7-9 adult males and 7-8 adult females.

\section{Behavioural data collection and construction of the hierarchy}

Behavioural data were collected by $\mathrm{CY}$ and five field assistants (MS09, MS10) and SH and one field assistant (MS11) from 0700 to 1900 hours. All data collectors were trained by CY and interobserver reliability was assessed repeatedly. All adult males in the group were subject to continuous focal animal observation of social and sexual behaviour (Altmann 1974), yielding a total of 902 focal hours during the three consecutive mating seasons (341 h MS09, 304 h MS10 and 257 h MS11). Data were collected using handheld HP IPAQ 114 series pocket PCs loaded with Pendragon Forms Version 5.1 (C Pendragon Software Cooperation, U.S.A.). With a randomised sequence each male was subject of one 40-min focal sampling session per observation day. 
Ejaculatory copulations were recorded and indicated by the occurrence of a distinct ejaculatory pause in pelvic thrusts (Kuester and Paul 1984) and/or the presence of fresh ejaculate around the female's genital area straight after the observed copulation had occurred. We did not include non-ejaculatory mounts in our analysis as Barbary macaques are single-mount breeders. Moreover, previous studies (Heistermann et al. 2008) found no discernible pattern of nonejaculatory mounts observed around the fertile phase compared to before and after this period but males concentrated their ejaculatory copulation rate around the fertile phase. In our data we found non-ejaculatory copulations to be evenly distributed across ranks and accounting for less than one third of all mounts observed. As ultimately only an ejaculatory copulation can lead to fertilisation we only include ejaculatory copulations in our analysis. Male inspection of a female's anogenital region was also recorded (tactile, olfactory or visual) as well as females' refusal to mate with males and incidents where a male would actively break-up and interfere in a male/female association (“Interference"). Consortship was defined as an exclusive malefemale dyad in which individuals remained within $10 \mathrm{~m}$ of each other, as well as coordinated movements when walking and was restricted to the mating season (Paul 1989; Bissonnette et al. 2011). Previous studies on the species only included a consortship with a minimum duration of 5 minutes so as not to overestimate male-female associations due to other factors such as feeding in close proximity (Heistermann et al. 2008; Bissonnette et al. 2011). Using the same methods, our study yielded an average consortship duration of $17.6 \pm 11.1$ minutes (mean \pm SD).

The dominance hierarchy was based on a total 620 male-male dyadic conflicts where a clear winner and loser of a conflict could be determined with no counter-aggression (MS09: 360; MS10: 73; MS11: 187). Following the methods of Young et al. (2013b) a separate hierarchy was constructed for each mating season using corrected Normalized David's Scores (de Vries et al. 2006). The hierarchies were significantly linear with adjusted linearity indices ( $h^{\prime}$ ) of 81.2 and a directional consistency index of 0.87 on average across the three seasons (assessed in MATMAN $^{\mathrm{TM}}$ 1.1.4 (Noldus (2003) following de Vries et al. (2006)). The proportion of known relationships was high (86.6\%), whereas the rate of counter-aggression $(4.8 \%)$, reversals $(0 \%)$, and two-way relationships (18.1\%) were low. Rank changes occurred between the alpha and the beta position, the $3^{\text {rd }}$ and $4^{\text {th }}$ ranking males had stable ranks across the study period, and one low ranking male emigrated out of the group at the end of both the MS09 and MS10 mating seasons. Males were classified, firstly by their ordinal rank position each mating season, and as high, mid- and low ranking using the following rationale: the Pandit/van Schaik coalition model (Pandit and van Schaik 2003) predicts that the targets of levelling coalitions will be the topranked or just below top, so males ranked $1^{\text {st }}$ and $2^{\text {nd }}$ were classified as high ranking. Leaving the remaining 7 (MS09) and 6 (MS10\MS11) males to be classified as mid- and low ranking. So males 
3-5 were classified as mid-ranking and males ranked $6^{\text {th }}$ and below were classified as low ranking.

\section{Female attractivity}

Anogenital swelling size was assessed visually using a 3 point scale adapted from the methods of Aujard et al. (1998) and Heistermann et al. (1996). All data collectors were trained by CY and swelling size was confirmed by at least two observers each day. For each swelling cycle, the maximum swelling period was calculated, as the 6 day window (day -2 to day -7 ) from detumescence. Using two of the three mating seasons the probability of fertility was shown to be at its maximum and always above 0.5 during this 6-day window (Young et al. 2013b). A day during the mating season which had one or more females at maximum swelling was termed an "attractive day" and a female was defined as in her "attractive period" on each day of her maximum swelling period.

\section{Expected and observed mating success}

Firstly, we calculated the expected mating success based on the PoA model (Altmann 1962). This is the proportion of ejaculatory copulations each male is expected to gain on a given day dependant on the number of females in their attractive period and the male's rank, e.g. if only one female was in their attractive period on a given day the highest ranking male would be assigned all the copulations of that day (i.e. a proportion of 1 ) and all other males none. If two females were in her attractive period, the two highest ranking males would be assigned 0.5 each and all other males zero, etc. The total score for each rank and day was summed and divided by the total number of attractive days to give the expected proportion of copulations each rank position was expected to gain. The daily observed mating success equals the actual number of ejaculatory copulations each male gained on a given day divided by total number of ejaculatory copulations on that day for each attractive day. The observed mating success for each rank over the study period again was calculated by summing up the daily observed mating success for each rank and dividing it by the total number of the attractive days.

\section{Female behaviour}

The influence of female mating behaviour on male mating success was investigated based on the number of female initiated sexual encounters to males on attractive days (hereafter "female invites"). A female invite was defined as an approach by a female towards a male within 1.5 metres proximity followed by sexual behaviour (copulation, sexual inspection and/or consortship behaviour). For each attractive day we then calculated the hourly rate of female invites from the 
number of focal hours each male was observed each day. This hourly rate was then divided by the number of receptive females each day to give a daily value of female invites per male per day. We also examined an additional female sexual behaviour, female's refusal to mate with males. We found this behaviour to be rare in our study group (39 observations during female attractive periods, only $3.2 \%$ of all mounts observed) and therefore did not consider it in the analysis.

\section{Coalitionary behaviour}

A coalition was defined as a simultaneous aggression by two or more partners against a common target (Bercovitch 1988). Due to the rapid, often complex and subtle behaviours involved in coalitionary bouts data were collected using handheld Kodak ZX1 HD video cameras with the observer providing a full spoken commentary of the entire aggression as soon as an aggression began. Due to the open habitat and low density of ground vegetation as well as the conspicuous and noisy nature of coalitionary aggression the vast majority of bouts were observed from the start. This was possible because many polyadic conflicts occurred during focal animal protocols and because the second observer was constantly seeking out situations that may cause polyadic conflicts to occur. Videos and spoken records together were analysed post-hoc and either added to the focal protocol or ad libitum database. Behaviour was coded using the same protocol as during a focal follow. If the target was the last participant to show submission he was deemed the loser of the coalition. Data shown here only include coalitions of multiple allies against one target. We classified coalitions into three categories 1) "all-up", 2) "all-down", and 3) "bridging" (van Schaik et al. 2004a; 2006). Only coalitions which occurred in a sexual context were used for this analysis. Coalitions under a sexual context were defined as a coalition occurring during a focal follow where one of the coalition's participants also displayed male sexual behaviour (consortship or ejaculatory copulation) during the focal follow. The number of coalitions a male was an actor in each day was divided by the number of receptive females each day to give a daily value of the number of coalitions a male was an actor in. Coalitionary activity was not standardized for observation time as a male could join a coalition when not the focal subject and therefore could be an actor in a coalition at any time of day. Incidences of single males interfering in sexual encounters and breaking up a male/female dyad were rare (57 observations across the entire study period and 23 during female attractive periods) and therefore were also not considered in the analysis. The aggressor in the majority of these interactions (92\%) was one of the top three ranked males although males ranked $1-6$ were observed to interfere at least once. In total $91 \%$ of interferences were observed by a higher ranked male against a lower 
ranked male with a female. Individual interferences accounted for only $6.1 \%$ of all aggressive interactions in a sexual context.

\section{The cost of proximity to females}

In order to examine whether time in proximity (within $1.5 \mathrm{~m}$ ) of a female during her attractive period would increase a male's probability of becoming the target of a coalition, we calculated for each attractive day (see above), during focal follows, the time each male spent in proximity to the female during her attractive period. Additionally, for each male, we calculated the total focal time on each attractive day. We then calculated, for each attractive day and each male, (i) the number of coalitions each male received whilst in proximity to a female and (ii) the number of coalitions each male received on attractive days not in proximity to a female during her attractive period. Hourly values for each male each day were calculated for $i$ and $i$ and we then averaged the daily values over the entire study period to give a mean hourly rate for each rank position and mating season.

\section{Statistical analysis}

A Spearman rank test was used to test for correlation of male rank and mean observed mating success for each rank position. The proportion of coalitions a male was a target of each mating season was related to the proportion of female initiated sexual encounters each male received using a Pearson's correlation.

Two different indices were used to calculate the mating skew, firstly, the "lambda" index of mating skew (Kokko and Lindström 1997), which measures the overall skew regardless of the dominance rank. Lambda ranges from 0 (evenly distribution of mating) to 1 (completely skewed mating towards one male). Secondly, we used the binomial skew index (hereafter B index; Nonacs 2000; 2003), a positive B index indicates greater than expected skew while more equally distributed mating is indicated by a negative value. A null hypothesis of random mating within the group can be tested against the observed skew using the $B$ index $(B=0)$. We tested both the observed and the expected PoA model's mating skew using the above methods. Both the lambda and B index were calculated using the Skew Calculator 2003 (set to 10,000 permutations) available online at https://www.eeb.ucla.edu/Faculty/Nonacs/PI.html.

A Generalized Linear Mixed Model (GLMM; Baayen 2008) was used to examine how male mating success was influenced by male rank, female receptive synchrony, and the interaction of these two variables, coalitions in a sexual context, and female behaviour. The dependant variable, male mating success was measured on the basis of the number of observed ejaculatory 
copulations each male gained per day. We included total number of observed matings per day (log-transformed) as an offset variable in the model to control for variation in mating frequency due to the number of females available each day. Predictor variables were 1) a male's ordinal rank position each day, 2) the number of receptive females each day, 3) the daily rate of female invites received and 4) the daily number of coalitions as an ally in a sexual context. Male rank and female receptive synchrony were included as an interaction in the model to examine if the influence of male rank on mating success changed over the range of synchrony values. All predictor variables were z-transformed. Mating season and male identity were included as random factors.

We ran the GLMM's in R 2.14.0 (R Development Core Team 2012) using the function Imer of the R package Ime4 (Bates et al. 2012). GLMM's were fitted with Poisson error structure and log-link function and likelihood ratio tests were calculated using the R function anova. Significance of the individual fixed effects was determined based on the $X^{2}$ - and $p$-values provided by Imer. Assumptions about the lack of over dispersion were respected for the analyses and we checked whether collinearity was a potential problem by using variance inflation factors (VIF) (Field 2005). VIF's were derived using the function vif of the R-package car (Fox and Weisberg 2010) applied to a standard linear model excluding the random effects. VIF's which are not substantially greater than one and less than ten indicate that covariation between predictors is not a problem (Bowerman and O'Connell 1990; Mayers 1990), in our analysis the range was 1.00 - 1.06. Additionally, we examined the correlation between the predictor variables in the full model and found low correlations between all predictors, confirming the VIF analysis.

To investigate the costs of proximity to females we used a paired-samples t-test to compare the hourly rate males were coalitionary targets while in proximity versus not in proximity to a female during her attractive period. Where appropriate, we report mean values \pm standard deviation (SD). All statistical analysis were carried out using R 2.14.0 software (R Development Core Team 2012). The level of significance was set at $\alpha<0.05$.

\section{Results}

\section{Comparing expected and observed mating skew}

We examined the observed mating skew and that expected by the PoA model for each mating season. Observed male mating skew was low across all three mating seasons indicated by a relatively low lambda (MS09 $=0.20, \mathrm{MS10}=0.20$ and MS11 $=0.37$ ), but still significantly skewed (MS09: $\mathrm{B}=0.02, \mathrm{p}<0.01$; MS10: $\mathrm{B}=0.02, \mathrm{p}=0.02$; MS11: $\mathrm{B}=0.07, \mathrm{p}<0.01$ ) and therefore, mating did not occur at random. The lambda of expected male mating skew (MS09 $=0.58$, MS10 
$=0.69$ and MS11 $=0.78$ ) indicated greater skew than the observed mating skew and mating was not expected to occur at random (MS09: $B=0.29, p<0.01$; MS10: $B=0.40, p<0.01 ; M S 11$ : $B=$ $0.50, p<0.01)$. We found strong negative correlations between male ordinal rank and observed mating success in all three mating seasons (Spearman correlation: MSO9 $=\mathrm{N}=9, \rho=-0.84, p<$ $0.01 ; \mathrm{MS} 10=\mathrm{N}=8, \rho=-0.97, \mathrm{p}<0.01 ; \mathrm{MS} 11=\mathrm{N}=8, \rho=-0.97, \mathrm{p}<0.01$ ), with higher ranking males gaining a greater proportion of copulations than lower ranking males. However, the observed mating skew was lower than the expected skew, i.e. the distribution was more even with the two top ranking males having lower than expected by the PoA model and the remaining males having higher than expected mating success (Figure 3.1). All males were observed to gain matings (up to rank 9) which was much higher than the number of maximum number of synchronously receptive females (5).

Factors determining mating skew: rank, female synchrony, coalitions and female behaviour

Throughout all mating seasons at least one female was in her attractive period on the majority of observation days, $80.7 \pm 6.7 \%$ (mean \pm SD) ( $M S 09=75.0 \%, M S 10=88.0 \%, M S 11=79.0 \%$ ) and on average $1.8 \pm 0.5$ (mean $\pm \mathrm{SD}$ ) females were in their attractive period on a given attractive day $(\mathrm{MSO9}=2.3, \mathrm{MS10}=1.9, \mathrm{MS11}=1.3)$. The maximum number of synchronously attractive females observed on a given day was $5(\mathrm{MSO9}=5, \mathrm{MS10}=4, \mathrm{MS} 11=3)$.

We observed on average $1.7 \pm 0.6$ coalitions per day during the mating seasons and throughout the three mating seasons $74.7 \pm 9.5 \%$ of coalitions took place on attractive days. On attractive days on average $48.7 \pm 20.8 \%$ of all coalitions occurred in a sexual context (see methods for definition); these were the coalitions we consider below. Coalitions which occurred in a sexual context ('levelling') were most frequently bridging (45.0\%), followed by all-down (34.5\%) and the least frequently all-up (20.5\%). Coalition formation was variable between the three mating seasons in terms of the main ranks of the allies and targets involved. Overall, the main allies in coalitions were high and mid-ranked males. The three most frequent coalition allies in MS09 were the $1^{\text {st }}(37.7 \%), 5^{\text {th }}(20.5 \%)$ and $2^{\text {nd }}(14.9 \%)$ ranked males. In MS10 it was the $1^{\text {st }}(34.1 \%), 2^{\text {nd }}$ $(25.6 \%)$ and $3^{\text {rd }}(18.9 \%)$ and in MS11 it was the $4^{\text {th }}(35.6 \%), 2^{\text {nd }}(25.6 \%)$ and $6^{\text {th }}(14.3 \%)$ ranked males. The main targets were high and mid-ranked males. The three most frequent targets of coalitions in MSO9 were the $4^{\text {th }}(34.9 \%), 3^{\text {rd }}(24.6 \%)$ and $2^{\text {nd }}(12.6 \%)$ ranked males. In MS10 it was the $4^{\text {th }}(31.7 \%), 5^{\text {th }}(18.3 \%)$ and $3^{\text {rd }}(15.9 \%)$ and in MS11 it was the $1^{\text {st }}(55.0 \%), 3^{\text {rd }}(25.0 \%)$ and $4^{\text {th }}$ (6.3\%) ranked males. 


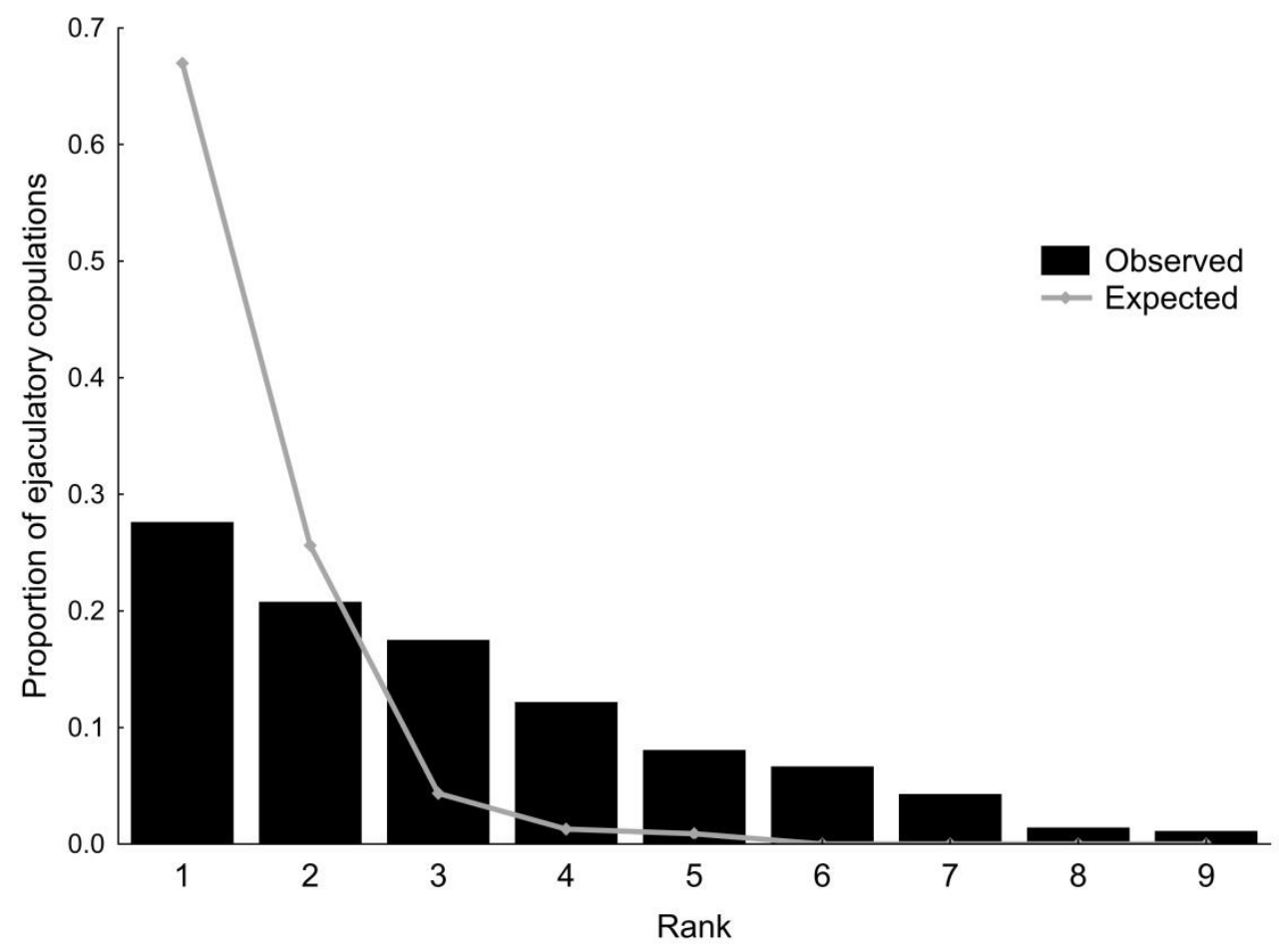

Figure 3.1: Expected and observed mating success. During the three mating seasons males occupied different rank positions and mean values are calculated for each rank position. The mean mating success was significantly negatively correlated with ordinal rank position (Spearman correlation, $N=9, \rho=-1, p$ $<0.01)$.

Females initiated $43.8 \% \pm 8.6$ (mean $\pm S D$ ) of all sexual encounters on attractive days. The ranks of males which females initiated sexual encounters with varied between mating seasons. The three most frequent recipients of female initiated sexual encounters were, in MSO9, the $3^{\text {rd }}$ $(21.0 \%), 5^{\text {th }}(18.5 \%)$ and $4^{\text {th }}(17.3 \%)$ ranked males. In the MS10 it was the $3^{\text {rd }}(32.4 \%), 4^{\text {th }}(17.6 \%)$ and $5^{\text {th }}(14.7 \%)$ and in the MS11 it was the $1^{\text {st }}(38.5 \%), 3^{\text {rd }}(19.2 \%)$ and $4^{\text {th }}(11.5 \%)$ ranked males. We found a positive relationship for all three mating seasons between the proportion of female initiated sexual encounters each male received with the proportion of coalitions a male was a target of with a trend for MS10 (Pearson's correlation: $r=0.61, d f=7, p=0.08$ ) and strong significant effect for MS09 (Pearson's correlation: $r=0.77, d f=7, p=0.01$ ) and MS11 (Pearson's correlation: $r=0.93, d f=7, p=<0.01$ ).

We ran a GLMM to investigate the influence of the four predictor variables and the interaction of male rank and female receptive synchrony on male mating success. Male ordinal rank explained the greatest amount of variance in the data and had a negative influence indicating 
that the higher males ranked the higher was their mating success on a given day (Table 3.1). The number of synchronous females per day positively influenced male mating success and it did so after controlling the response by the total number of copulations by all males and females observed that day as an offset term. This indicates that as female receptive synchrony increased many males' mating success also increased. The interaction between male rank and female receptive synchrony was not significant but the relationship with the response was positive which suggests that the effect of rank tends to decrease with increasing synchrony. Both of the main effects were significant, thus, not rank alone but also synchrony had an independent effect on male mating success.

A large proportion of variance in male mating success was explained by female behaviour, i.e. the number of times a male was approached by a female in a sexual context which may be interpreted as female preference (Figure 3.2). Another and independent part of the residual variance was explained by male coalitionary activity, i.e. the more often a male was an ally in a coalition against another male in a sexual context the higher his mating success was (Figure 3.3).

The cost of proximity to females

Across all males in the group we found that the rate of coalitionary attacks received was increased when the male was in proximity of a female in her attractive period (mean $=0.82 / \mathrm{hr}$.) compared to when no such female was close (mean $=0.01 / \mathrm{hr}$; paired t-test: $t=3.53, \mathrm{df}=8, \mathrm{p}<$ $0.01)$. 
Table 3.1: GLMM Poisson regression results for the relationship between observed mating success (controlling for number of matings per day) and male ordinal rank, the number of synchronously receptive female, the interaction of male rank and female receptive synchrony, female initiated sexual encounters and a male being an ally in a coalition $(N=701)$.

\begin{tabular}{lcccc}
\hline Independent variable & $\begin{array}{l}\text { GLMM } \\
\text { estimate }\end{array}$ & SE & $Z$ & $P(>|Z|)$ \\
\hline $\begin{array}{l}\text { Intercept } \\
\text { Female initiated sexual } \\
\text { encounters }\end{array}$ & -1.360 & 0.092 & -14.771 & $<0.01$ \\
$\begin{array}{l}\text { Male rank } \\
\text { Number of synchronous }\end{array}$ & -0.412 & 0.038 & -5.150 & $<0.01$ \\
$\begin{array}{l}\text { females } \\
\text { Ally in a coalition }\end{array}$ & 0.111 & 0.080 & 6.412 & $<0.01$ \\
$\begin{array}{l}\text { Interaction of rank and } \\
\text { synchrony }\end{array}$ & 0.063 & 0.041 & 2.693 & 0.01 \\
\hline
\end{tabular}

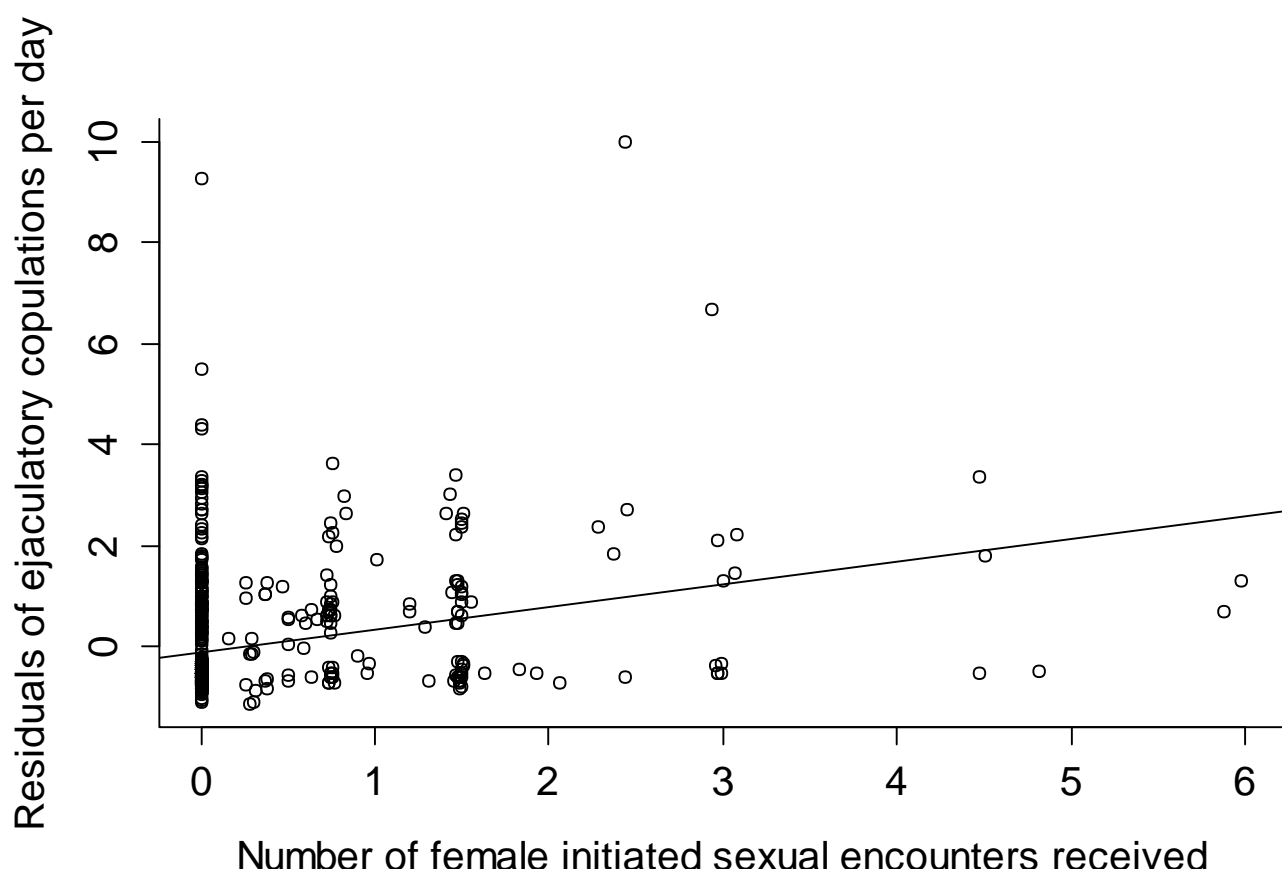

Figure 3.2: Relationship between the observed mating success and the number female initiated sexual encounters per hour a male received per hour (controlling for number of receptive females, see methods). The y-axis represents the residuals of male ejaculatory copulation rate (controlling for number of matings per day) obtained from a GLMM including male rank, female receptive synchrony and number of coalitions as an actor as fixed factors and male identity and mating season as random factors. 


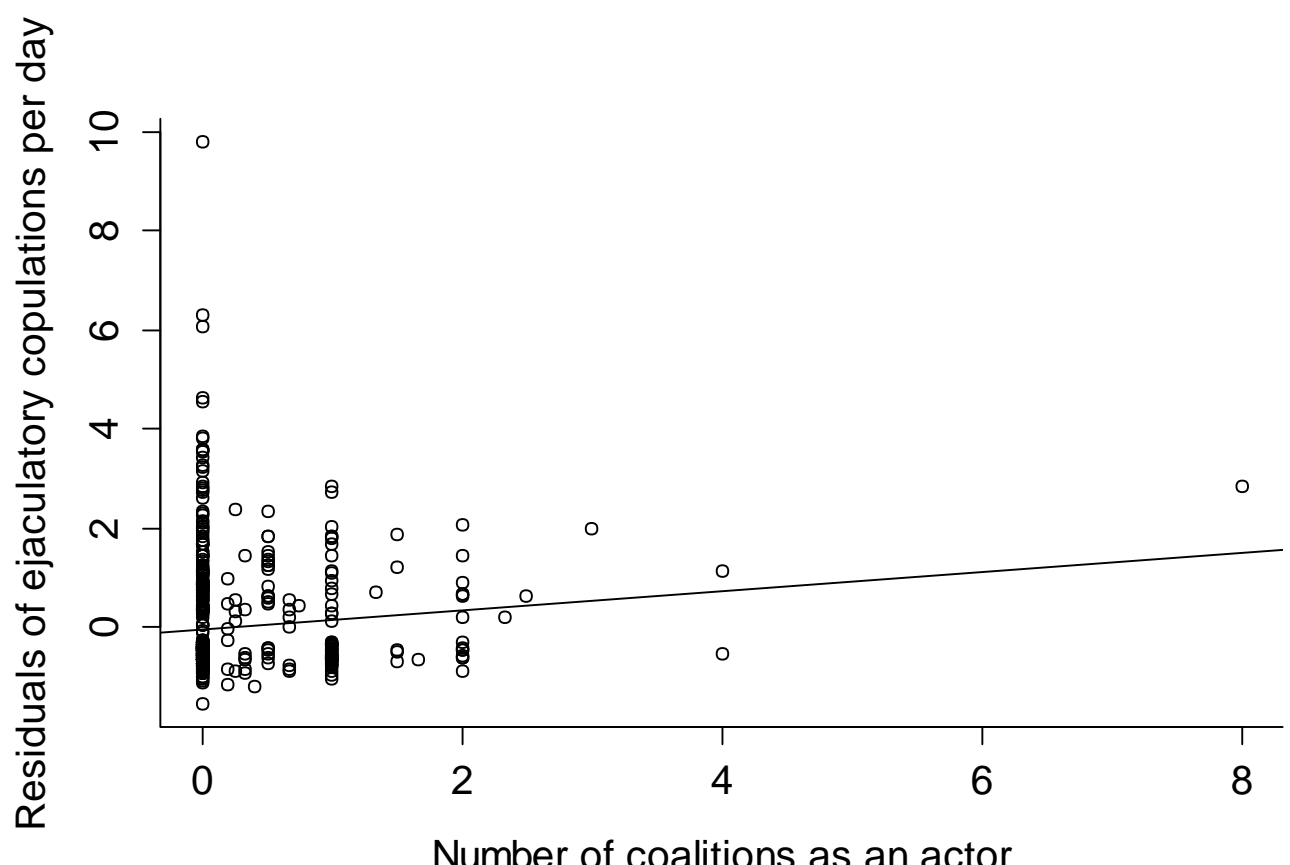

Figure 3.3: Relationship between the observed mating success and the number of coalitions a male was an actor in (controlling for number of receptive females, see methods). The y-axis represents the residuals of male ejaculatory copulation rate (controlling for number of matings per day) obtained from a GLMM including male rank, female receptive synchrony and number female initiated sexual encounters a male received as fixed factors and male identity and mating season as random factors. We re-ran the GLMM model without the outlier and the results of the GLMM did not change.

\section{Discussion}

Our results show that mating in Barbary macaques was skewed up the hierarchy with the highest ranking males gaining the greatest mating success across consecutive mating seasons. The observed mating skew by rank was very low though and much lower than predicted by the PoA model. The assumptions of the PoA model are based on rhesus macaque (Macaca mulatta) biology (Altmann 1962). Thus, the large difference between observed and predicted mating distribution may not be surprising in a species without extended male mate-guarding regulating access to females. We found, however, a crucial aspect of the model to be relevant in Barbary macaques, i.e. female reproductive synchrony significantly affected male mating success.

The PoA model has been tested in various primate (see Kutsukake and Nunn 2006) and other mammalian species (Hirotani 1994; Say et al. 2001; Engh et al. 2002). Support for the PoA model, however, is mixed. It has been shown to be a good predictor of mating success in many species (Bulger 1993; Weingrill et al. 2000; Soltis et al. 2001; Alberts et al. 2003; Weingrill et al. 2003; 
Takahashi 2004; Setchell et al. 2005; Boesch et al. 2006; Wroblewski et al. 2009), but not so in other studies (Say et al. 2001; Engh et al. 2002; Widdig et al. 2004; Hayakawa 2008; NewtonFisher et al. 2010; Bissonnette et al. 2011; Dubuc et al. 2011; this study). This mixed support may be due to varying measures of female receptivity (Alberts et al. 2006; Wroblewski et al. 2009; Henzi et al. 2010) as the information males can infer about female reproductive state varies greatly between taxa. Alternatively, the strict assumptions of the model may not be met in species where extended periods of mate-guarding may be less pronounced, for example, hyenas, Crocuta crocuta (Engh et al. 2002), domestic cats, Felis catus L. (Say et al. 2001) and Barbary macaques (Bissonnette et al. 2011; this study) leading to a poor fit. However, rhesus macaque mating and reproductive skew also showed a poor fit (Widdig et al. 2004; Dubuc et al. 2011) although they are the species the model was originally designed for, and thus should meet the model's assumptions. We argue that even without extended male mate-guarding of receptive females, high ranking males can use their status to increase their access to females and that female receptive synchrony will affect the efficiency of this strategy. Therefore, both male rank and female receptive synchrony can still play a large role in influencing male mating success even in species with an absence of extended mate-guarding periods.

By first examining the roles of male rank and female synchrony we set the stage for exploring additional factors influencing male mating success, using multivariate analysis. We found a large amount of the residual variance in male mating success to be explained by female behaviour and male coalitionary activity. Females were found to initiate sexual interactions primarily with midranking males which may explain why mid-ranking males accumulated larger numbers of matings than one may expect in a small group where a mean of only 1.7 and rarely more than 3 females were mating in synchrony. We investigated whether the inability of high ranking males to monopolize access to these females may result from levelling coalitions breaking-up consortships and reallocating matings to lower ranking males as previously described for semifree ranging Barbary macaques (Bissonnette et al. 2011). In support of this idea the risk of being target of an aggressive coalitionary attack increased with the time a male spent with attractive females. But male coalitionary behaviour did not target the top/just below top ranking male as the Pandit/van Schaik coalition model (Pandit and van Schaik 2003) predicted and as found in the previous study (Bissonnette et al. 2011). In our study group that was smaller and lacked the large number of old and very old males of the previous study, coalitions were mainly bridging between high and lower ranking males against mid-ranking targets. Upon closer inspection the discrepancy between studies only concerns the ranks of allies and targets but not the context of the coalitionary attacks. The targets of both studies were those males that received most attention from the females in the group which were the top ranking males in the previous and 
the mid-ranking males in our study. Thus, coalitions in our study may have functioned mainly to counterbalance against the effect of female behaviour and the participation of low ranking males in these coalitions may explain their non-zero share of matings.

As mentioned in the introduction, male mating skew can be influenced not only by female mate choice and male coalition formation but also by several other factors, including energetic costs of mate-guarding, number of competitors in a group, stability of the dominance hierarchy, male sexual coercion, and invasions by non-resident males (reviewed in Alberts 2012). These factors vary in the degree to which they affect mating success in different species, for example, in some species there is less potential for female mate choice or an absence of male coalition formation (e.g. in mandrills (Mandrillus sphinx) (Setchell et al. 2005) or chacma baboons (Papio hamadryas ursinus) (Weingrill et al. 2003)). In species with extended mate-guarding periods the energetic costs of mate-guarding may significantly reduce mating skew (Packer 1979; Rasmussen 1985; Alberts et al. 1996; Coltman et al. 1997; Mainguy and Côté 2008; Pelletier et al. 2009; Schubert et al. 2009). In our study, there was little evidence of male sexual coercion, male-female associations being broken up by individual males, and invasions from non-resident males. Thus, these factors are not considered to have affected male mating skew.

Demographic and individual male characteristics may additionally influence male mating skew in primate groups. In our study, one male left the group during two mating seasons for one month creating possible instability of the hierarchy, which may have affected the alpha male's ability to monopolise access to females (Cowlishaw and Dunbar 1991). High ranking males may prefer multiparous over nulliparous females (Newton-Fisher et al. 2010) or concentrate mating to conception swellings (Wroblewski et al. 2009) but in our study all females were multiparous and high ranking males mate equally often during conception and non-conception swellings (Young et al. 2013b). Males far apart in age are expected to have larger power differentials and therefore males living in groups with a wider age range may engage in alternative strategies, such as coalition formation, to compensate for reduced fighting ability whereas males closer in age are more likely to compete one-on-one (Alberts et al. 2003). Age differences in wild populations are likely to be smaller than in free-ranging conditions; however mating skew was similar in the study on semi-free ranging Barbary macaques exhibiting a wide age range (Bissonnette et al. 2011) suggesting that age-span alone may not play a prominent role in influencing male mating skew.

An additional factor considered to influence male mating success is the costs of extended mate guarding. Barbary macaque males, however, may not suffer energetic costs of extended mateguarding as consortship duration is very short but short-term associations with females may still 
be costly. Males who associated with females, in our study, were frequent targets of coalitionary aggression. Increased aggression leads to greater risk of injury (Paul and Kuester 1988; Kuester and Paul 1992), increased energy expenditure during the contest (Muller and Wrangham 2004), and increased physiological stress (Wallner et al. 1999; Ostner et al. 2008a), as well as a reduction in future mating opportunities (Bercovitch 1988; Bissonnette et al. 2011). Thus, males may incur costs unrelated to extended mate-guarding but with similar detrimental outcomes to a male's mating success.

Female mating behaviour can play a major role affecting male mating skew by influencing monopolisability of females in their attractive period. Female mating behaviour can either increase (Janson 1984; Boinski 1987; Brauch et al. 2008) or decrease (Strier 1996; Widdig et al. 2004) male mating skew depending on whether females select one or many mating partners. Japanese macaque (Macaca fuscata) females show preferences for mating with lower ranked males suggesting that even in these highly despotic macaques female behaviour can play a role (Soltis et al. 1997). In some species female refusal to mate with certain males may indicate female preferences for certain partners (Huffman 1987), but refusal rate in our study was too low to add important information to our results. Female mating behaviour in our study did seem to affect male mating success with females showing preference towards the alpha male, as well as mid-ranking males ( $3^{\text {rd }}$ and $4^{\text {th }}$ rank). By doing so this would reduce the mating share distributed towards the top ranked males whilst simultaneously increasing the share of the midranked males. Possible explanations as to why females select these mid-ranked males may be due to the prospective remaining tenure length of the alpha male, with females investing in males moving up the hierarchy as those will be the best protector for their future offspring (Alberts et al. 2003; van Noordwijk and van Schaik 2004; Clarke et al. 2009).

Females may also engage in frequent sexual encounters with immigrant males (Berghänel et al. 2010; Bissonnette et al. 2011; but see rhesus macaques, Manson 1995), who may soon rise in rank and also increase genetic diversity. In our study group, the position of the mid-ranked males did not increase over the three year study period suggesting that neither novelty nor future rank increase would explain their attractiveness to females. Alternatively, females may have been merely attempting to reduce future risks of infanticide by increasing paternity confusion across the highest ranked males (van Schaik et al. 2000; van Noordwijk and van Schaik 2004), or selecting males for other factors such as MHC compatibility and quality (Setchell and Huchard 2012), inbreeding avoidance (Zeh and Zeh 2001) or post-copulatory sperm competition (Dixson 1998). 
In addition to female behaviour, a male's coalitionary activity was found to have a pronounced independent influence on male mating success in our study. The Pandit/van Schaik model (Pandit and van Schaik 2003) predicts that coalitions in a levelling context should be all-up coalitions against top or males just below top rank or be bridging coalitions formed between kin. These all-up levelling coalitions have been observed in baboons (Packer 1979; Bercovitch 1988; Noë and Sluijter 1990; 1995) and semi-free ranging Barbary macaques (Bissonnette et al. 2011). In our study high and lower ranking males teamed up to form bridging coalitions against midranking males. van Schaik and colleagues (2004a; 2006) predicted that bridging coalitions should only be profitable for the higher ranked partner if the allies are kin (with males dispersing from their natal groups (Kuester and Paul 1999; Modolo et al. 2008) the chances of co-resident males being kin are reduced). The rationale being that males compete for a non-shareable resource and the dominant male should have the greatest fighting abilities and be able to monopolise access to females without cooperation of other individuals (van Schaik et al. 2004a; 2006). However, if the alpha male cannot completely control mating access, for example due to female behaviour as in this study, and as such matings are being concentrated towards other group members, bridging coalitions may be a viable option. By forming bridging coalitions with lower ranked males, the high ranked male may not necessarily increase his mating success directly but will disrupt future mating opportunities of the mid-ranked males and prevent individual females to concentrate mating in a particular mid-ranking rival. Coalitionary activity has been shown to result in a consortship change-over in approximately half of the coalitions observed in studies in baboons and macaques (Bercovitch 1988; Noë 1992; Bissonnette et al. 2011) with one ally gaining a mating opportunity with the contested female directly after the coalitionary bout and not necessarily the highest ranked ally. However, coalitions can also be utilized to intimidate other group members (Berghänel et al. 2011b) as the dyadic dominance relationship between the target and each of the allies can be affected by the joint aggression. In future dyadic contests between the target and one of the allies over access to a receptive female the ally's chance of success is increased due to their increased external power (Berghänel et al. 2011a).Therefore, by forming coalitions a male can limit the mating success of other group members which indirectly increases their own mating success.

Overall, we found, even in a species without extended male mate-guarding, that both male hierarchal position and female receptive synchrony are important predictors of male mating success. We suggest that both factors should be considered in future studies looking to examine mating/reproductive skew (Port and Kappeler 2010), not only in primates but mammalian multimale, multi-female groups with variable female receptive synchrony. Importantly, the information available to males about female reproductive state (the extent of this information 
varies widely among taxa) needs to be available for an assessment of the predicted PoA distribution. In addition to male rank and female receptive synchrony, several other factors can influence male mating success in mammalian species and recent advances in multivariate statistics allow the influence of these factors to be considered independently. In Barbary macaques, we found that although male mating success is skewed up the hierarchy it is poorly predicted by expectations of the PoA model. Both male coalitionary aggression and female behaviour acted to limit the alpha males' capacity to monopolise access to females in our study. Together with other results (Schülke et al. 2010) this suggests that cooperation increases male reproductive success in male dispersal species. The PoA model may provide a good framework with which to investigate male mating success in species with variable female receptive synchrony, even in the absence of extended male mate-guarding periods. A multi-level approach to examine male mating \reproductive success, firstly looking at a species fit to the PoA model and then the additional factors which independently affect mating skew may advance our understanding of the relationship between male dominance rank and mating\reproductive success in different taxa, and, in turn, male and female reproductive strategies overall.

\section{Acknowledgments}

The authors are grateful to Professor Mohamed Qarro (Ecole Nationale Forestière d'Ingénieurs, Morocco) for his support in the field and the Haut Commissariat aux Eaux et Forêts et à la Lutte Contre la Désertification of Morocco for research permission. We would also like to thank Michael Madole, Dave Thomas, Sofia Santos, Maria Thunström, Tom Smith, Josephine Msindai and Carrie Miller for assistance in the field and Laëtitia Maréchal, Cédric Girard-Buttoz, Ines Fürtbauer and Mathias Franz for helpful comments. Furthermore, we would like to thank Dr. Elise Huchard and two anonymous referees for useful comments to significantly improve the manuscript. Financial support was provided by the Max Planck Society, the Christian Vogel Fonds and Georg-August University Göttingen through funds from the German Initiative of Excellence. 


\title{
Chapter 4
}

\section{Beyond mutual tolerance - how males form coalitions against group rivals and the Pandit/van Schaik coalition model}

\author{
Christopher Young ${ }^{1}$, Oliver Schülke ${ }^{2^{*}} \&$ Julia Ostner ${ }^{1^{*}}$
}

${ }^{1}$ Primate Social Evolution Group, Courant Research Centre Evolution of Social Behaviour, GeorgAugust University Göttingen, Kellnerweg 6, 37077, Germany.

${ }^{2}$ Courant Research Centre Evolution of Social Behaviour, Georg-August University Göttingen, Kellnerweg 6, 37077, Germany.

*equal contribution as last authors

Behaviour, in revision 


\section{Abstract}

In group living animals, there is pronounced variation in the formation and function of cooperation between males in the form of coalitionary aggression. van Schaik and colleagues (2003; 2004a) developed a mathematical model to predict the probability and profitability of different coalition types between group-living male primates ("PvS model"). According to the model, coalitions are classified into five types dependent on the ranks of the participants and the context of the aggression. The main factor determining the type of coalitions expected to evolve in groups is contest potential, an estimate of the monopolisability of females by the alpha male. We examined the model using groups of Assamese (Macaca assamensis) and Barbary (Macaca sylvanus) macaques under varying contest potentials. We measured contest potential on a species-specific basis dependent on the information males can infer about female reproductive state. By examining coalition formation in different populations and different species, but in the same groups over time, we gained a full range of contest potentials and were able to show the strengths and weaknesses of the PvS model for coalition formation. We discuss why our results do not fully fit the predictions of the PvS model, such as differing costs and benefits of coalition formation and alternative factors driving partner choice and target selection not considered by the model. Finally, we suggest possible improvements to the model and provide information on how best to test the current predictions of the PVS model.

Keywords

Coalition model; male sociality; cooperation; macaque. 


\section{Introduction}

Cooperation in the form of coalition formation has been widely observed across many groupliving non-human primate taxa (reviewed by Smith et al. 2010), several non-primate mammalian taxa (Zabel et al. 1992; Connor et al. 1999; de Villiers et al. 2003; Jennings et al. 2009), and is a ancestral trait of hunter-gatherer human societies (Boehm 1993; Boehm 1999). Coalitions can be defined as a simultaneous aggression by two or more partners against a common target in a competitive or aggressive context (Bercovitch 1988; de Waal and Harcourt 1992). Coalitions can occur within groups or between groups and be formed between males, females and between the sexes. Classical socio-ecological reasoning makes the formation of male-male within group coalitions most puzzling (van Hooff and van Schaik 1992) as males living in social groups compete in a zero-sum game for a non-shareable resource, namely conceptions (Trivers 1972; CluttonBrock and Parker 1992).

Within-group coalitions among males have been shown to serve several different functions, such as maintenance of the allies' position in the dominance hierarchy (rank-maintaining, Berman et al. 2007), increasing the allies' rank position (rank-changing, Nishida 1983; Schülke et al. 2010) or destabilizing dyadic dominance relationships (Berghänel et al. 2011b). In addition to functions related to males' social status, coalitions may also be used as means to induce either scramble competition (Berghänel et al. 2010) or dominant males' access to receptive females (levelling, Packer 1979; Bercovitch 1988; Noë and Sluijter 1990; 1995; Bissonnette et al. 2011). Coalition formation may be opportunistic with the short term goal of increasing subordinates' access to a female by breaking up consortships (see levelling above), or be based on the strength of partners' social relationships yielding patterns of coalition formation that are relatively stable over longer time periods and potentially serve several functions though time (Schülke et al. 2010; Gilby et al. 2012). Support in agonistic conflicts may also buy mating privileges and in that way work in the mid-term (Duffy et al. 2007).

Several theoretical and mathematical models have been designed to investigate different aspects of coalition formation. These models have looked at various factors which may influence coalition formation from an individual's motivation to interfere in a dyadic contest (Dugatkin 1998; Johnstone and Dugatkin 2000), the optimal size of alliances (Whitehead and Connor 2005), how encounter frequency influences a dyad's tendency to form coalitions in fission-fusion societies (Connor and Whitehead 2005), relatedness between females (Broom et al. 2009), to access to food resources, a non-zero sum situation (Stamatopoulos et al. 2009). The applicability of many of these models to the case of within-group male coalitions seems limited, because female models assume philopatric allies and a sharable resource which females compete over, 
whereas most primate males are dispersing and compete in a zero-sum game. Two further models looked at male primates examining payoff structures between allies (Noë 1990) and the combined fighting abilities of allies (Noë 1994) but these models consider only one specific type of coalitions (e.g. within group all-up levelling coalitions).

A decade ago van Schaik and colleagues published a mathematical model, the Pandit/van Schaik model (hereafter the PvS model; Pandit and van Schaik 2003; van Schaik et al. 2004a; 2006) to predict the occurrence of different rank constellations and function types of within group coalitions between male primates. The model invoked the feasibility (i.e. will the allies ever be strong enough to win against the target) and the profitability (i.e. will the reproductive success of the allies be increased by coalition formation) of coalitions as the crucial determinants of their evolution. The modelling exercise revealed that, given several assumptions, the entire variation in constellation and type of coalitions could be predicted from one factor only, i.e. the environmental potential for despotism or the potential for contest competition. Here, we investigated the occurrence of coalition constellations and types within and across groups of Barbary macaques (Macaca sylvanus) and across species by including data on male Assamese macaques (Macaca assamensis) in relation to the PvS model. In doing so, we evaluate the scope of the model and provide a roadmap for future formal tests and potential improvements of the PvS model.

Following Chapais (1995), Pandit and van Schaik (2003) classified coalitions into three constellations based on the ranks of the allies in the coalition: 1) all-up, where both allies are lower ranking than the target, 2) all-down, where both allies are higher ranking than the target, and 3) bridging, where the target is ranked between the allies. These coalition constellations were further categorized into types by their function, being either 'rank-changing' when they affect participants' rank position, or 'levelling', when they reduce the inequality in the distribution of matings across ranks (van Schaik et al. 2004a). The model assumes that individual pay-offs are not directly related to male fighting ability but are mediated by dominance rank instead. Pandit and van Schaik (2003) invoked the Priority of Access model (hereafter the PoA model; Altmann 1962) to describe differences in payoffs of rank neighbours. The parameter " $\beta$ " in PvS model is the exponent of the negative exponential function derived from the PoA model, i.e. it describes the size of these differences or the skewedness of the payoff curve over dominance rank. $\beta$ is conceptualized as the environmental potential for despotism which is equivalent to the degree of contest competition over receptive females. Variation in $\beta$ alone predicted the constellation and type of coalitions primate males engaged in both in 
mathematical modelling as well as in a preliminary survey of published empirical data (van Schaik et al. 2004a).

Different ways of estimating the contest potential $\beta$ from paternity distribution have been discussed (van Schaik et al. 2006). Preliminary tests of the model are based on the proportion of offspring sired by the alpha male (van Schaik et al. 2004a) which may be problematic because it is not only a measure of contest potential $\beta$ but also a post-hoc measure influenced by the outcome of coalitions. Here we argue that expected mating success of the alpha male as predicted from the PoA model may be a more accurate measure of $\beta$. As it more closely resembles a measure of the environmental potential for despotism and is not affected by the coalition's males formed to level the payoff distribution. The alpha male mating success as predicted by the PoA model behaves like $\beta$ in the sense that it "tends to be reduced when (1) the number of females in the group increases, (2) the duration of each oestrous period increases, and (3) the temporal overlap between oestrous periods increases, usually because matings are seasonal" (Pandit \& van Schaik, 2003, p. 162, Nunn 1999b; Kutsukake and Nunn 2006; Ostner et al. 2008b; Gogarten and Koenig 2012). Since the basic logic of the PoA model is that a male can only monopolize access to one female at a time and that the rank of the most subordinate male getting access equals the number of synchronously receptive females, deriving predictions form the PoA model requires information about what males "know" about female reproductive state. However, the extent to which males can infer information about female fertility varies greatly between species, ovulation may be concealed from males (Heistermann et al. 2001; Fürtbauer et al. 2011a) or mating can be extended into gestation (Gordon et al. 1991; Gust 1994; Engelhardt et al. 2007; Lu et al. 2012; Young et al. 2013b). Thus, calculations of male contest potential and $\beta$ should be based on species-specific estimates of the PoA model using the fertility information available to males (Young et al. 2013a; Sukmak et al. submitted).

Specifically, the PvS model predicts (summary in Table 4.1) at medium contest potential, that allup rank changing coalitions should occur, targeting the top rankers with the allies ranking just below top and coalitions of small size. Additionally, all-up levelling coalitions are also expected at mid-low contest potential which will target the same males but the allies will be low to midranked and a small-large size is expected depending on the costs involved in coalition formation (Pandit and van Schaik 2003; van Schaik et al. 2004a). Although the authors noted post-hoc that the model may slightly underestimate the $\beta$ value up to which all-up coalitions may occur and they may be observed at slightly higher levels of $\beta$ (van Schaik et al. 2004a). Bridging rankchanging coalitions are predicted to only be profitable for the higher ranked partner if their ally is kin as they can gain indirect fitness benefits and therefore should occur between genetic 
relatives, involve males in the higher regions of the hierarchy and increase in frequency as $\beta$ increases. Bridging levelling coalitions are also expected to occur only among related males but are otherwise expected to occur under a wide range of conditions in the direct conflict over access to a receptive female. All-down coalitions are predicted to occur wherever all-up and bridging coalitions occur and to have a defensive function but are not explicitly modelled in the PvS model.

So far the PVS model has only been examined in a few species under free-ranging conditions (Higham and Maestripieri 2010; Bissonnette et al. 2011). Although these studies found support for the PvS model each was limited in examining which coalitions occurred at only one specific contest potential. Here we investigate the PvS model across different years in the same groups, different groups of the same species, and closely related species with contrasting levels of contest potential during mating seasons. Across two species of macaques we observed all five coalition types described by the PvS model. Here we will examine how the predictions of the PvS model compare to the actual observed constellations. More specifically, we will examine the rank of the target and allies of the coalitions as well as the size and contest potential with which each coalition type occurred. We also draw on published data on coalition formation in Barbary macaques (Berghänel et al. 2010; Bissonnette et al. 2011) in order to examine coalition formation across a wider range of group sizes and therefore more variable partner choice.

Table 4.1: Each coalition constellation and function described by the PvS model is shown along with the predicted rank of the coalition target, allies and coalition size. Adapted from Bissonnette (2009) and van Schaik et al. (2006).

\begin{tabular}{llllll}
\hline $\begin{array}{l}\text { Coalition } \\
\text { constellation }\end{array}$ & Function & Rank of target & Rank of allies & Coalition size & $\begin{array}{l}\text { Predicted } \\
\text { occurance }\end{array}$ \\
\hline Bridging & Rank-changing & Near top & $\begin{array}{l}\text { Top and low } \\
\text { relative }\end{array}$ & Small & $\begin{array}{l}\text { More with } \\
\text { increasing } \beta\end{array}$ \\
Bridging & Levelling & Anywhere & Variable & Variable & Variable \\
All-up & Rank-changing & $\begin{array}{l}\text { Top or near } \\
\text { top }\end{array}$ & Just below top & Small & $\beta<0.5$ \\
All-up & Levelling & $\begin{array}{l}\text { Top or near } \\
\text { top }\end{array}$ & Mid and low & Small-large & $\beta<0.5$ \\
All-down & $\begin{array}{l}\text { Rank- } \\
\text { protecting }\end{array}$ & Low-rankers & $\begin{array}{l}\text { Top and near } \\
\text { top }\end{array}$ & Probably small & $\begin{array}{l}\text { If levelling and } \\
\text { bridigng occur }\end{array}$ \\
\hline
\end{tabular}




\section{Methods}

\section{Study sites and data collection}

Data for this study were collected from two species of macaques, Barbary and Assamese macaques. Firstly, Barbary macaque data were collected on two wild, unprovisioned, fully habituated groups ('Green' (Gn), 'Scarlet' (Sc) group) living in a deciduous cedar and oak forest in the Middle-Atlas Mountains of Morocco $\left(33^{\circ} 24^{\prime} 9 \mathrm{~N}\right.$ and $\left.005^{\circ} 12^{\prime} 9 \mathrm{~W}\right)$. The study population breeds seasonally with a mating season from September to December and a corresponding birth season from March to May (Young et al. 2013b). Data were collected during two mating seasons (Gn09: Oct -Dec 2009, Gn10: Sep - Dec 2010, Sc10 Sep - Dec 2010). The mating season was defined as the period of the first to the last observed ejaculatory copulation during either focal or ad libitum data collection. Behavioural data were collected by $\mathrm{CY}$ and five field assistants from 0700 to 1900 hours, for details see Young et al. (2013). A total of 820 focal hours were collected during the mating seasons ( 341 h Gn09, 304 h Gn10 and 175 h Sc10). Group composition was consistent through-out the study period; the Gn group consisted of 9-8 males and 7 females while the Sc group consisted of 6 males and 8 females.

Secondly, Assamese macaque behavioural data were collected on a wild, fully habituated, unprovisioned group ('As1') from the dry evergreen forest study area Huai Mai Sot Yai situated in the Phu Khieo Wildlife Sanctuary (16 $05-35^{\prime} \mathrm{N}$ and $\left.101^{\circ} 20-55^{\prime} \mathrm{E}\right)$ in Thailand. The sanctuary is part of the approximately 6,500 $\mathrm{km}^{2}$ Western Isaan Forest Complex (Koenig et al. 2004). The mating season lasts from October to January/February and the subsequent birth season occurs between April and June/July (Fürtbauer et al. 2010). Data used here were collected between October 2006 and September 2008, including two mating seasons. Data were collected via focal animal observation (2,825 hours) supplemented with ad libitum sampling on agonistic interactions from 0600 to 1800 hours by a team of experienced field assistants, for details see Ostner et al. (2008a) and Schülke et al. (2010). The group consisted of 12-13 males and 11-12 females during the study period.

This study was conducted completely non-invasively and under the permission of the authorities of Thailand and Morocco. We adhered to the Guidelines of the Use of Animals in Research, the legal requirements of Thailand and Morocco, and the guidelines of the involved institutes. 


\section{Formation of dominance hierarchy}

For both species the dominance hierarchy was constructed based on male-male dyadic conflicts where a clear winner and loser of a conflict could be determined with no counter-aggression. A separate hierarchy was constructed for each mating season and each species using corrected Normalized David's Scores (de Vries et al. 2006) to give a continuous measure of dominance. For Gn09, Gn10 and Sc10 see the methods of Young et al. (2013b) and for As1 see the methods of Ostner et al. (2008a).

\section{Calculating the contest potential}

Contest potential was determined by calculating the expected mating success of the alpha male for each group and each mating season using the PoA model (Altmann 1962). In order to do so we had to determine species-specific estimates of the PoA model using the fertility information available to males, previously determined using hormonal analysis for both Assamese (Fürtbauer et al. 2011a; 2011b) and Barbary macaques (Young et al. 2013b). For Assamese macaques, males mate with females frequently from the beginning of the mating season until conception has occurred, when mating frequency drops substantially (Fürtbauer et al. 2011a; 2011b). Conception was determined via counting back from the day of infant birth using a previously determined gestation length (Fürtbauer et al. 2010). All females that conceived in a mating season were considered receptive up until the day of conception because females do not show behavioural estruses interspersed with periods without matings. This created a considerable overlap of receptive periods when calculating the expected mating distribution (Sukmak et al. submitted).

We have previously shown (Young et al. 2013b) that Barbary macaque males mate most frequently around the period of maximum anogenital swelling and cannot distinguish between swelling periods in which ovulation occurs and those which occur during pregnancy (Young et al. 2013b). As such, all maximum swelling periods were used to estimate the expected male mating success with each day of the maximum swelling period for each female being considered a "receptive day", i.e. a day the female is expected to mate, and on these days each female was termed "receptive". The mean duration of maximum swelling periods in our study was $7.14 \pm$ 3.63 days (mean $\pm S D, N=45$ ). Due to the species differences in information males can infer about female reproductive state we used these different measures of female receptivity for each species in order to obtain empirical estimates of $\beta$.

The PoA model estimates the proportion of ejaculatory copulations each male is expected to gain on a given day depending on the number of simultaneously receptive females and the 
male's rank, e.g. if only one female was receptive on a given day the highest ranking male would be assigned all the copulations of that day (i.e. a proportion of 1 ) and all other males zero matings. If two females were receptive, the two highest ranking males would be assigned 0.5 each and all other males zero, etc. The total score for each rank and day was summed and divided by the total number of receptive days to give the expected proportion of copulations each rank position was predicted to gain. The contest potential for each mating season was the proportion of matings expected for the alpha male (see introduction).

The PvS model describes five categories of $\beta$ from very high to very low (Pandit and van Schaik 2003; van Schaik et al. 2004a; 2006) and using this rationale we divided the contest potential into five categories as follows: very high $\beta>0.81$, high $\beta=0.61-0.80$, medium $\beta=0.41-0.60$, low $\beta=0.21-0.40$ and very low $\beta<0.20$. The $\beta$ values for each group and mating season were derived from the PoA models expected mating success as calculated above.

\section{Coalition definitions}

A coalition was defined as a simultaneous aggression by two or more partners against a common target, Figure 4.1 (Bercovitch 1988; de Waal and Harcourt 1992). Coalitions were recorded during both focal follows and ad libitum as they occurred and behaviour during coalitions was coded using the same protocol as during a focal follow. We classified coalitions into three categories 1) 'all-up', 2) 'all-down', and 3) 'bridging' (van Schaik et al. 2004a; van Schaik et al. 2006). Coalitions under a sexual context were defined as a coalition occurring during a focal follow where one of the coalition's participants also displayed male sexual behaviour (consortship or ejaculatory copulation) during the focal follow. These coalitions under a sexual context were then defined as 'levelling' for Barbary macaques. For Assamese macaques the context (here "sexual" (levelling) or "other") of each coalition was recorded at the time the coalition occurred in the field. This resulted in coalitions either having a direct sexual context and thus, being levelling or a non-sexual context and being assigned a rank related function. $\mathrm{A}$ note of caution, we interpret coalition function post-hoc however, it may be that the true intention of the coalition prior to the event was different. By clearly categorising levelling coalitions as those which directly occur under a sexual context we hope to limit this error as much as possible. The mean coalition size was calculated from the mean number of allies in all coalitions observed during that mating season. All other analyses of coalition formation consider only coalitions of two allies against one target. 


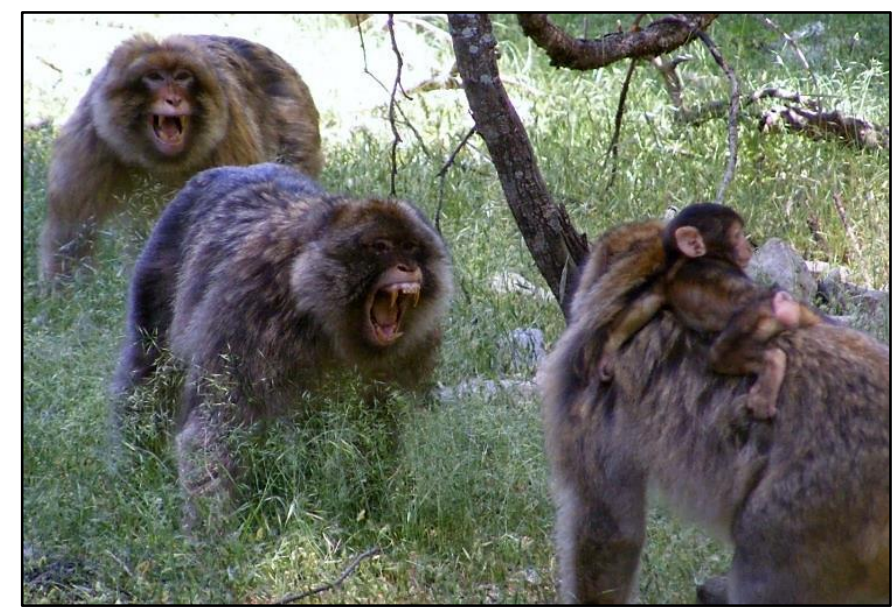

Figure 4.1: Two males simultaneously aggressing a common target (on the right) in a coalitionary attack. The common target is another male who is carrying an infant (a common behaviour in male Barbary macaques).

\section{Statistical analysis}

A Pearson's correlation test was used to relate percentage of all-up coalitions with the percentage of all-down coalitions observed during each mating season. The percentages were calculated from all coalitions observed during each mating season (see Table 4.2), including bridging coalitions. We used proportions of the total number of coalitions observed to control for the variable coalition number during each mating season, for example Sc10 we observed 35 and Gn09 we observed 177 coalitions. Statistical analysis were carried out using R 2.14.0 software (R Development Core Team 2011). The level of significance was set at $\alpha<0.05$.

\section{Results}

We found evidence for the formation of all five coalition types in Assamese and Barbary macaques, at both differing and similar contest potentials. Below we provide descriptions of each coalition type found and the predictions from the PvS model as to when this coalition type should occur. These details are summarised in Table 4.2. Data on wild Assamese and Barbary macaques were supplemented with published data from a group of free-ranging Barbary macaques at Affenberg, Salem, Germany, that was observed during two mating seasons (Sa06 and Sa08) using similar methods (Bissonnette et al. 2009a; 2011; Berghänel et al. 2010; 2011a, 2011b). 


\section{Contest potential}

Contest potential $(\beta)$ was high in the Barbary macaque $\mathrm{Gn} 10$ and Sc10 mating seasons with the proportion of matings expected for the alpha male being 0.76 (Gn10) and 0.78 (Sc10). Medium contest potential was found in the Barbary macaque Gn09 $(\beta=0.50)$ mating season and low contest potential for the Assamese macaque As1 mating seasons $(\beta=0.34)$. In the studies of free-ranging Barbary macaques contest potential was measured as the days that females were considered attractive to males based on maximum swelling size (For details see Berghänel et al. 2010; Bissonnette et al. 2011). For these studies medium contest potential was also observed (Sa08 = 0.53; Berghänel et al. 2010, Sa06 = 0.47; Bissonnette et al. 2011).

Table 4.2: For each mating season the total number of coalitions observed (two actors vs. one target) is shown as well as the number of all-up, all-down, bridging coalitions observed and the mean size of the actors of the coalitions (all coalitions observed). In parenthesis is the number of coalitions under a sexual context (levelling). ${ }^{1}$ Berghänel et al. (2010) and ${ }^{2}$ Bissonnette et al. (2011).

\begin{tabular}{lcccccc}
\hline $\begin{array}{l}\text { Group/ } \\
\text { period }\end{array}$ & $\begin{array}{l}\text { Contest } \\
\text { potential }\end{array}$ & \multicolumn{1}{c}{ Total } & All-up & All-down & Bridging & Mean size \\
\hline Sc10 & 0.78 & 35 & $9(4)$ & $19(15)$ & $7(4)$ & 2.18 \\
Gn10 & 0.76 & 82 & $10(4)$ & $45(25)$ & $27(14)$ & 2.19 \\
Gn09 & 0.50 & 177 & $15(5)$ & $63(19)$ & $98(37)$ & 2.24 \\
Sa08 & 0.53 & 155 & $64(20)$ & $29(2)$ & $62(4)$ & 2.3 \\
Sa06 & 0.47 & 90 & $73(25)$ & $8(0)$ & $29(13)$ & 2.0 \\
As1 & 0.34 & 99 & $5(0)$ & $70(1)$ & $24(0)$ & 2.18 \\
\hline
\end{tabular}

High contest potential

At high contest potential bridging rank-changing and perhaps bridging levelling coalitions are expected. All-up coalitions are not expected to occur at $\beta$ levels much higher than 0.5 however; we found all-up rank-changing and all-up levelling coalitions in $\mathrm{Gn} 10(\beta=0.76)$, Table 4.2. As well as all-up rank-changing coalitions in Gn10, we also observed many bridging levelling coalitions, which are predicted to occur across a wide range of contest potentials but only among related allies (van Schaik et al. 2006). In our study, these bridging coalitions were formed by alpha and beta males with lower ranked partners (mainly $5^{\text {th }}$ and below) and targeted mid-ranked males. Males ranked $3^{\text {rd }}$ and $4^{\text {th }}$ in the hierarchy (the main mid-ranked targets, targets of $50 \%$ of coalitions) gained $14 \%$ and $11 \%$ more matings than predicted by the PoA model (Young et al. 
2013a). Females also initiated sexual encounters most frequently with these males suggesting that these males could have had even higher mating success.

In the Sc10 mating season the majority of coalitions were all-down (55\%) with only $25 \%$ all-up and $20 \%$ bridging at a high contest potential, Table 4.2. All-up coalitions, again at a high $\beta$ value (0.78), targeted the alpha male on all-but-one occasion and $60 \%$ of all-up coalitions were formed by the $2^{\text {nd }}$ and $3^{\text {rd }}$ ranked male against the alpha male. Only $45 \%$ of the all-up coalitions against the alpha male were levelling, thus the majority were rank-changing, although rank positions of all three males remained stable during the study. The all-down coalitions mainly targeted successful mid-low ranked males gaining higher than expected mating success (33\% of matings in total).

The PvS model predicts all-down protective coalitions to be always feasible and to occur whenever there are offensive all-up and bridging coalitions occurring. In support of this we found across the six seasons that the percentage of all-down coalitions increased as the percentage of all-up coalitions decreased (Pearson's correlation, $r=-0.85$, $d f=4, p=0.03$; Figure $4.2)$.

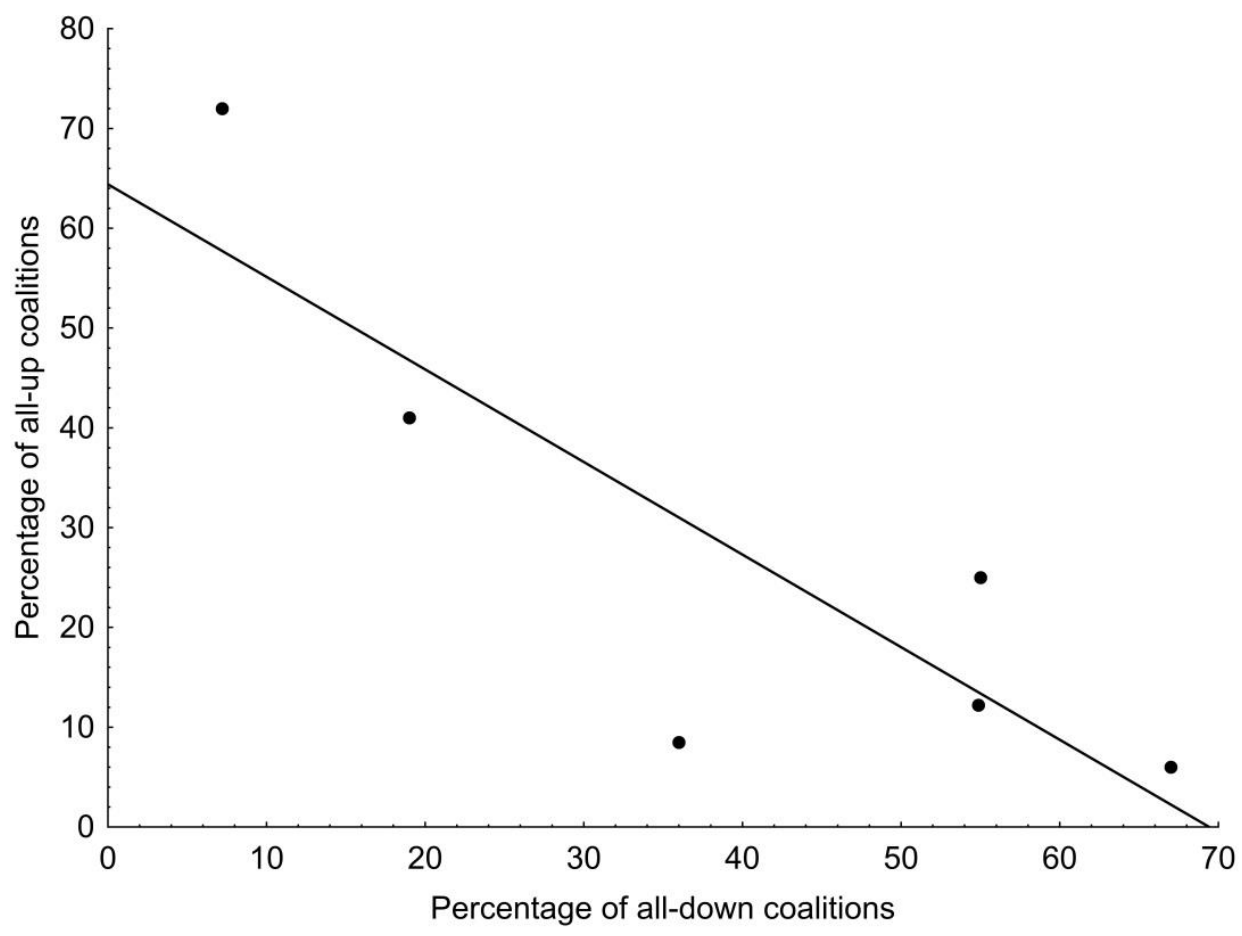

Figure 4.2: Percentage of all-up against all-down coalitions comparing all mating seasons, including the Sc08 (Berghänel et al. 2010) and Sc06 (Bissonnette et al. 2011) mating seasons. The percentage of each coalition type was calculated from the total number of coalitions occurring each mating season, including bridging coalitions. 


\section{Medium contest potential}

At medium contest potential, the PVS model predicts that all-up rank-changing and all-up levelling coalitions should occur, both targeting the top rankers. The size for rank-changing should be small with the allies ranking just below top. Whereas allies are predicted to be low to mid-ranked and size should be small-large for levelling coalitions. Medium contest potential was observed in Barbary macaques during the Gn09, Sa06 and Sa08 mating seasons. In the Gn09 mating season we observed the same all-up rank-changing coalitions as in Gn10, i.e. at high contest. Additionally, we observed frequent bridging levelling coalitions as with Gn10. Despite the marked difference in contest potential the observed coalition types and constellations were very similar for Gn09 and Gn10.

We now provide a brief description of the rank-changing coalitions occurring during both the Gn10 and Gn09 mating seasons. A low ranked male (ND, $5^{\text {th }}$ ranked) would leave the group during the mating season for one month ( $33 \%$ of the mating season and was observed on the periphery of other groups). Upon his return to the group he was the main coalition partner of the $2^{\text {nd }}$ ranked male $(\mathrm{OZ})$ and these two males frequently formed all-up rank-changing coalitions against the top ranked male (AR) who consequently dropped to second rank with the higher ranked ally (OZ) assuming the alpha position. The rank position of ND did not change, remaining $5^{\text {th }}$ ranked before and after his return. OZ and ND continued to form bridging coalitions against mid-ranked males as well as against the former alpha male ( $45 \%$ initiated by OZ). These may have been unsuccessful coalitions to increase the rank of ND or alternatively defensive coalitions ("bridging rank-maintaining") to protect the newly acquired alpha status of OZ from retaliatory aggression of the ousted male. When ND was present during the Gn09 and Gn10 mating seasons there were 82 coalitions formed by OZ against AR ( 0.21 coalitions per hour) and of these 44 (54\%, 0.12 per hour) were with ND, while when ND was absent there were only 12 coalitions involving $\mathrm{OZ}$ against $\mathrm{AR}$ ( 0.07 coalitions per hour), as $A R$ was alpha during this time these were all-up coalitions.

All-up levelling coalitions were also reported by Bissonnette et al. (2011) for the Sa06 and by Berghänel et al. (2010) for the Sa08 mating seasons. In Sa06 a total of 90 coalitions were observed of which 73 were all-up (Table 4.2) and 25 of these had a direct levelling function (Bissonnette et al. 2011). In comparison to all-up coalitions there was fewer bridging (29) and alldown were infrequent (8). During Sa08, 155 coalitions were observed and the majority were also all-up (64) with 20 of these occurring with a direct levelling function. Bridging coalitions were equally as frequent with 62 observed and 29 all-down coalitions were observed. In the Sa06, coalitions primarily targeted high ranked males or those with the greatest mating success. 
Conversely, during Sa08 78\% of coalitions targeted 5 young immigrant mid-ranking males and the coalition partners were mainly low ranking older post-prime males. Interestingly, in both studies the main targets of coalitions were the same individuals but their rank positions had changed between the Sa06 and Sa08 and changing the coalition constellations observed.

Low contest potential

The PvS model predicts that at low contest potential all-up levelling coalitions should still occur. In As1 we observed only $6 \%$ of coalitions to be directed all-up while $67 \%$ were all-down and $27 \%$ were bridging coalitions. In total 99 coalitions were observed and only one bridging coalition occurred under a sexual context (Table 4.2). Although no levelling was observed males who frequently formed coalitions increased their future rank position, whereas those males who did not form coalitions dropped or never rose in rank (Schülke et al. 2010). This suggests that males formed bridging rank-changing and all-up rank-changing coalitions over long-time periods. High ranked males consorted females and consort changeover resulted from a high ranking male losing interest or moving on to another female (Ostner et al. 2011) but not due to coalitionary aggression.

\section{Discussion}

In male macaques from two species, we found all possible rank constellations combined with both types of coalitions described by the PvS model. Our study supported several predictions of the PvS model. The frequency of all-up levelling coalitions at $\beta$ above 0.5 was very low. The number of all-down defensive coalitions observed increased with decreasing frequency of all-up coalitions across our study groups. At medium contest potential we observed all-up rankchanging and all-up levelling coalitions in Barbary macaques. Some of our results, however, are in stark contrast to predictions from the PvS model. We found clear evidence for all-up rankchanging coalitions at high contest potential. All-up levelling coalitions were predicted at midlow contest potential but we found no evidence at low contest. Moreover, we observed bridging rank-changing coalitions at low contest potential which are predicted to be more prevalent the higher the contest potential. The ranks of targets and allies were not well predicted for all-up rank-changing and bridging levelling coalitions. Observations of the same group after rankchanges had occurred revealed characteristics of the targets other than their dominance rank (age and immigration status) predicted coalition constellation. Altogether bridging coalitions occurred at a high frequency in species with male dispersal which may be surprising as they are predicted to be formed only by closely related males. By examining coalition formation in the 
same groups over time, different populations and different species, we were able to show the strengths and weaknesses in the PvS model for male within group coalition formation.

Selecting the best estimate of contest potential is essential in order to examine the predictions of the PvS model. van Schaik and colleagues (Pandit and van Schaik 2003; van Schaik et al. 2004a; 2006) suggest possible estimators of contest potential within groups, for example, the alpha male's share of paternity or the ratio of paternity of the beta to the alpha male, however sample size can dramatically affect these estimates (Alberts et al. 2006). We would propose utilizing the PoA model and the predictions of expected mating success, based on speciesspecific estimates of female receptivity based on information males can infer about female reproductive state (also see Alberts et al. 2006; Wroblewski et al. 2009; Henzi et al. 2010). Additionally, as van Schaik and colleagues (2006) acknowledge, post-hoc estimates of $\beta$ may incur an additional inaccuracy. The mere threat of coalitionary attacks against high rankers may intimidate them into not fulfilling their monopolisation potential to avoid future coalitionary attacks. Thus, all post-hoc estimates of $\beta$ may carry an unavoidable error. Furthermore, the PvS model predicts each coalition constellation to occur over a wide range of $\beta$ values. The combination of these factors makes estimating $\beta$ accurately very difficult and as a result deriving clear testable predictions from the PVS model is problematic. van Schaik and colleagues (2004a) also acknowledge that all-up rank-changing coalitions were observed at higher $\beta$ than expected in their review of the empirical data and postulate that this may be due to inflated $\beta$ in captive conditions. In this study we find all-up rank-changing coalitions at high values of $\beta$ also in wild populations suggesting this mismatch between the model and empirical data may not entirely be a remnant of captivity.

Here we examine two seasonally breeding species however; the PvS model is still applicable to more aseasonal species as calculating $\beta$ via the PoA model accounts for female receptive synchrony. We would expect that as mating becomes more aseasonal the potential for monopoly of females by the alpha male would increase as less females are likely to be receptive concurrently. Thus, $\beta$ would most likely be at the higher end of the scale and eventually if complete monopoly would occur and with high power asymmetries between males coalition formation would no longer be viable.

The levelling function of coalitions is predicted to be most frequently expressed during all-up coalitions as lower ranked males cooperate to break up consorts of higher ranked males, who are expected to gain the greatest proportion of mating success (Packer 1979; Bercovitch 1988; Noë and Sluijter 1990; 1995; Bissonnette et al. 2011). However, alternative reproductive strategies, such as female mate choice, can alter predicted mating skew away from the highest 
ranked males (Young et al. 2013a). Bridging levelling coalitions could be utilized to counteract this (see Gn09\Gn10). This is basically a violation of the model's assumption that payoffs are a strict function of rank. Successful rivals would be targeted irrespective of the targets' rank as they are gaining a greater share of matings. The subordinate ally may gain some additional matings but from the perspective of the alpha male it is better to dilute the matings across the group than have it concentrated in one main rival (Young et al. 2013a). The alpha male should be able to break up consorts of mid-ranking males but in the intense competitive mating season, coalition formation can be a viable option to reduce the risk of escalation of the contest and subsequent injury (Bissonnette et al. 2009a). The allies may not directly gain temporal access to the female from the coalition but they will reduce future mating opportunities for the target and indirectly increase their own share of matings.

At the low contest potential of Assamese macaques the rarity of levelling coalitions and the observation of bridging rank-changing coalitions were not predicted by the PvS model. For Assamese macaques female fertility is concealed (Fürtbauer et al. 2011a; 2011b) and therefore the immediate temporal benefit of breaking up a consort using opportunistic coalitions may be very low. The probability of the female being fertile when the consort is broken up is low so immediate short-term access may not benefit the male and the risk of targeting a high ranked male may outweigh the immediate benefit gained. Despite low skew, the alpha male does gain the highest mating success however, therefore it still pays to attain alpha position (Ostner et al. 2011). Males may utilize rank-changing coalitions to attain higher future rank positions. Once the rank-change has occurred several protective coalitions maybe required to defend the new higher status and therefore require a regular coalition partner (van Schaik et al. 2006). These coalition partners must not defect during aggression against a usually higher ranked target as defection would leave the other partner in a vulnerable position (Ostner and Schülke submitted). Affiliation and social bonding between males may act to mediate coalition formation with males selecting a regular partner (Schülke et al. 2010; Ostner and Schülke submitted). For lower ranked males in these groups it may pay to invest in an alliance with delayed benefits, with the potential for both males to rise in rank over time and take the top rank positions in the future (Schülke et al. 2010).

Males, who regularly form coalitions together in both macaques (Schülke et al. 2010; Berghänel et al. 2011a) and chimpanzees, Pan troglodytes (Mitani 2006b; Mitani 2009), also have stronger social bonds, but are often not maternal kin (Langergraber et al. 2007; Mitani 2009; Schülke et al. 2010). Thus, in species where male-male affiliation is frequent and differentiated social bonds can form between males the PvS model may miss a vital element (social bonding) in the 
calculation of costs and benefits. Feasibility in the PVS model in determined by rank position of males as only males of specific rank positions would have the intrinsic fighting abilities to form a successful coalition against certain targets (Pandit and van Schaik 2003; van Schaik et al. 2004a; 2006). However, strongly bonded males may be distributed unevenly across the dominance hierarchy and this may explain the high number of bridging coalitions in our study groups. In male dispersing species we may expect familiarity and kinship to play a less important role than in philopatric male societies but unfortunately, determining relatedness of Barbary macaque males was beyond the scope of this study. Additionally, a regular coalitionary partner can act to increase a males intrinsic power even in the absence of the partner and function to intimidate opponents and reduce the probability of aggression from higher rank individuals (Berghänel et al. 2011b). Males can use a build-up of frequent coalitionary attacks and the mere threat of a male soliciting support may counteract the disproportionate fighting abilities higher rank individuals (similar to random acts of aggression by dominants; Silk 2002b).

In species where social interactions, and thus social relationships, between males are rare, such as savannah baboons, Papio sp. (Packer 1979; Bercovitch 1988; Noë and Sluijter 1990; 1995), rhesus, M. mulatta (Higham and Maestripieri 2010) and Japanese macaques, M. fuscata (Kutsukake and Hasegawa 2005) the predictions of the PvS model seem more likely to hold. Both levelling coalitions in baboons and rank-changing coalitions in macaques were found to follow the predictions of the PVS model. Where differentiated social bonds are lacking males may simply judge the fighting abilities of other males and support the male with the greatest strength or weigh-up the strength of a coalition against them and whether escalating contest is worth the risk (Bissonnette et al. 2009a). Further investigation is required to determine if males are opportunistically supporting the most likely winner or show longer-term partner preferences.

The PvS model predicted coalition size would increase as the contest potential decreased but our study did not fit this pattern with coalition size being consistently small (Table 4.2). Limited partner availability and competition for a non-sharable resource (in the case of levelling coalitions) were given as potential reasons for smaller than expected coalition size (Pandit and van Schaik 2003; Bissonnette 2009) but even with large variation in group sizes and therefore number of available partners (6 - 13 males in our data from wild Barbary and Assamese macaques and up to 23 in Sa06/Sa08) coalition size did not vary. Additionally, many coalitions were not of a direct levelling function and therefore, access to a limited resource is unlikely to explain the lack of variation. We would suggest that the lack of variation in coalition size may be related to male selecting partners with strong social bonds as males are likely to have only a few partners with strong alliances in a group. Alternatively, Pandit and van Schaik (2003) suggest that 
adding costs to the PvS model would result in predictions of smaller coalition formation. Refinement of the model to include cost effects may help to resolve this issue.

Finally, we would like to suggest some possible additions and improvements to the PvS model which we think could further advance the current model's predictability of within group malemale coalition formation in non-human primates. 1) The PvS model in its current state may only be applicable to species where males compete directly for immediate benefits and access to resources. The model may have to be altered to accommodate long-term payoffs, mediated by social relationships, or where alternative factors such as female mate choice play a large role altering the targets of coalitions. 2) Feasibility is derived from rank position in the model and based on the assumption that a higher ranked individual will have greater fighting abilities than those ranked lower. However, a dynamic aspect is lacking in the model to account for the rise in rank of males utilizing rank-changing coalitions. A rank change results in male " $\mathrm{A}$ " climbing above male " $B$ " but the fighting abilities of $A$ and $B$ remain the same as before. By including a dynamic aspect to the model over time may accommodate the behavioural flexibility of primates as they ascend and descend in rank. 3) Coalition constellations are currently predicted to occur on a presence $\backslash a b s e n c e$ basis at differing contest potentials and not at specific frequencies. However, several coalition types can occur at one contest potential at varying frequencies so refinement of the current mathematical model to predict coalition frequencies may provide more testable predictions. These improvements to the mathematical model may lead to clearer predictions of which coalition constellation\type is most likely to occur across different contest potentials.

The PvS model provides a framework with which to test certain aspects of coalition formation in its current state, particularly in species where social bonds and affiliation between males are lacking. Additional considerations to examine the model in the future would include using an accurate estimate of contest potential. This would be derived from accurate estimates of what males know about female reproductive state on a species-specific basis, at the time of data collection. Secondly, information of male relatedness within a group would be vital to test the predictions of bridging coalitions and kin related costs and benefits of coalition formation. Precise assessments of the current testable predictions of the PVS model are difficult to interpret without such information. Furthermore, by examining contest potential in species where coalition formation is rare or absent as well as those where coalitions are frequent (such as our study species) may shed light on the mechanisms behind coalition formation. 


\section{Acknowledgements}

We would like to thank Erin Riley and Katharine Jack for the invitation to contribute to this special issue. Additionally, we thank three anonymous reviewers for comments to significantly improve the manuscript. The authors are grateful to Bonaventura Majolo (University of Lincoln) and Professor Mohamed Qarro (Ecole Nationale Forestière d'Ingénieurs, Morocco) for their support in the field and the Haut Commissariat aux Eaux et Forêts et à la Lutte Contre la Désertification of Morocco for research permission. We also thank the National Research Council of Thailand (NRCT) and the Department of National Parks, Wildlife and Plant Conservation (DNP) for the permission to conduct research on Assamese macaques and for all the support granted (permit 0004.3/3618, current permit 0002.3/2647). We are grateful to Kitti Kreetiyutanont, Mongkul Kumsuk, Kanjana Nitaya, Jarupol Prabnasuk, and currently Thanee Vongnak (Phu Khieo Wildlife Sanctuary) for their cooperation and permission to carry out this study and to Naris Bhumpakphan, Wicharn Eiadthong (Kasetsart University) and Warren Brockelman (Mahidol University) for support and cooperation. We thank Andreas Koenig and Carola Borries (Stony Brook University), who developed the field site at Huai Mai Sot Yai. We would also like to thank M. Madole, D. Thomas, S. Santos, M. Thunström, T. Smith and J. Msindai for assistance with data collection on Barbary macaques and D. Bootros, N. Bualeng, A. Chunchaen, I. Fürtbauer, M. Heesen, R. Intalo, N. Juntuch, S. Jumrudwong, M. Karlstetter, Thipusa Kilawit, S. Macdonald, W. Nueorngshiyos, D. Pesek, N. Ponganan, S. Rogahn, P. Saaisawasthikul, M. Swagemakers, B. Whitman and T. Wisate for data collection on Assamese macaques. We thank Annie Bissonnette for fruitful discussions on coalition formation in Barbary macaques and Laëtitia Maréchal and Cédric Girard-Buttoz for helpful comments on the manuscript. Financial support was provided by the Max Planck Society, the Christian Vogel Fonds and Georg-August University Göttingen through funds from the German Initiative of Excellence. 


\section{Chapter 5}

\section{Male social bonds predict partner choice in cooperative aggression in wild Barbary macaques}

Christopher Young ${ }^{1}$, Bonaventura Majolo ${ }^{2}$, Oliver Schülke ${ }^{3^{*}}$ \& Julia Ostner ${ }^{1^{*}}$

${ }^{1}$ Primate Social Evolution Group, Courant Research Centre Evolution of Social Behaviour, GeorgAugust University Göttingen, Kellnerweg 6, 37077, Germany.

${ }^{2}$ School of Psychology, University of Lincoln, Brayford Pool, Lincoln, LN6 7TS, U.K.

${ }^{3}$ Courant Research Centre Evolution of Social Behaviour, Georg-August University Göttingen, Kellnerweg 6, 37077, Germany.

*equal contribution as last authors 


\section{Abstract}

Aggressive cooperation in coalitions against co-resident males have been shown to increase male mating success and indirectly via effects on dominance rank also paternity success. Two mechanisms have been proposed to guide partner choice for coalition formation. Accordingly, males either base partner choice on past experience, i.e. chose partners they share a close social bond with. Or partner choice is more opportunistic and depends mainly on partner availability at the time the coalition is formed and on the expected success as predicted by allies' and target's fighting ability. Different studies on male Barbary macaques (Macaca sylvanus) have produced support for both of the mechanisms but crucial assumptions and predictions remained untested. Here we aim at investigating whether Barbary macaque male affiliative relationships qualify as social bonds and at testing the relative support for previously untested predictions from both mechanisms. We observed two groups of wild Barbary macaques in Morocco for over 2,000 hours and recorded the identity of males recruited to join in a coalition along with the identity of all bystanders and the target of the coalition. We found that males formed differentiated and equitable social bonds that were stable over more than two years and three mating seasons. We found that males did not base their partner choice on a simple rule of thumb derived from opportunistic criteria. Instead, males chose to recruit that male from the audience they shared the closest bond with. The probability that a bystander rejected a recruitment attempt was predicted by both the dominance rank of the target and the strength of the social bond between the bystander and the recruiter. Together these results add to the growing body of evidence that social bonds are an important functional tool in guiding partner choice, ultimately leading to fitness benefits, even for the dispersing sex.

Keywords

Social bonds; coalition; male-male competition; recruitment; bystander; Macaca sy/vanus. 


\section{Introduction}

Animals living in relatively stable social groups often have to act concurrently cooperatively and competitively with other group members, competing for resources whilst establishing social relationships with both kin and non-kin (Cords 1997; Silk 2005; Silk 2007; Massen et al. 2010). Strong female-female social relationships have been shown to carry fitness benefits for the individuals involved, such as reduced physiological stress levels (Engh et al. 2006; Crockford et al. 2008; Wittig et al. 2008), enhanced infant survival (Silk 2003; Silk et al. 2009), and increased reproductive success (Armitage and Schwartz 2000; Cameron et al. 2009; Frère et al. 2010; Silk et al. 2010b; Wey and Blumstein 2012). Recent evidence suggests that among males differentiated social bonds are more wide-spread than originally thought (Silk 1994; Perry 1998; Connor et al. 2001; Duffy et al. 2007; Mitani 2009; Fraser and Bugnyar 2010; Schülke et al. 2010; Berghänel et al. 2011a; Teichroeb et al. 2013; Ostner and Schülke submitted) which is surprising owing to males' competition for an indivisible resource, i.e. fertilisations (van Hooff and van Schaik 1994). Here we investigated whether the adaptive benefit of strong social bonds accrues from coalition formation against other males or whether coalition formation is guided by more immediate criteria about the expected success of the coalition.

Mammalian males can cooperate with cohorts through aggressive coalition formation (Bercovitch 1988; de Waal and Harcourt 1992), as seen in several taxa (reviewed by Smith et al. 2010). Coalitions generally occur under two main contexts, firstly levelling coalitions (Pandit and van Schaik 2003) where males attempt to level mating skew and gain immediate benefits (Bercovitch 1988; Noë and Sluijter 1990; Bissonnette et al. 2011). Secondly, males can utilize rank changing coalitions (van Schaik et al. 2004a; 2006) either using coalitionary aggression to increase or maintain the rank of one or both partners (Riss and Goodall 1977; Higham and Maestripieri 2010; Schülke et al. 2010). Reproductive success tends to be skewed up the hierarchy (Cowlishaw and Dunbar 1991; Ellis 1995; Majolo et al. 2012) and thus attaining increased rank position through coalitionary aggression can be a long-term reproductive strategy, requiring a stable, reliable coalition partner, which may be mediated by strong social bonds between the partners (Schülke et al. 2010; Ostner and Schülke submitted). However, as well as strength of social bonds alternative explanations have been proposed to explain partner recruitment during coalition formation, such as attitudinal partner choice (Noë 1990; 1992; 1994), maximising feasibility (Bissonnette et al. 2009a) and spatial proximity (Noë and Sluijter 1995; Puga-Gonzalez et al. 2009; Hemelrijk and Puga-Gonzalez 2012), discussed below. 
Males should recruit coalitionary partners with whom they are likely to maximise their success and minimise their costs (van Schaik et al. 2004a; 2006) thus, partners with the greatest strength/fighting abilities should be selected and the intrinsic power should out-weigh that of the target (Harcourt 1992; Noë 1992; Pandit and van Schaik 2003; van Schaik et al. 2004a; 2006; Bissonnette et al. 2009a). Males may use cognitively simple "rules of thumb" when recruiting a coalition partner and simply recruit the highest ranking male to maximise their likelihood of success, maximising feasibility (Bissonnette et al. 2009a), rather than using more complex cognitively taxing criteria such as knowledge of third party rank or social relationships (Silk 1999; Perry et al. 2004; Range and Noe 2005; Schino et al. 2006; reviewed by Cheney 2011). Alternatively, males may base their recruitment decisions on their previous interactions and experiences with potential allies (Noë 1992; 1994; Noë and Völkl 2013). We label this mechanism "attitudinal partner choice" to allow for the possibility that partner choice is guided by market forces that maintain long-term asymmetries between partners (Noë 1992; 1994; Noë and Völkl 2013) whereas "attitudinal reciprocity" (de Waal 2000) evokes past behaviour as the major predictor of partner choice but assumes equability between partners for giving and receiving of behaviours over the longer term. However, recent studies have shown that males may support coalition partners based on social bonding and affiliation rather than merely on the hierarchal position and relative fighting abilities of the conspecifics available (Silk 1994; Mitani et al. 2002; Watts 2002; Perry et al. 2004; Schülke et al. 2010; Berghänel et al. 2011a; Gilby et al. 2012).

Social bond strength and coalition frequency between dyads has been correlated in several species (Silk 1994; Connor et al. 2001; Mitani et al. 2002; Watts 2002; Perry et al. 2004; Berghänel et al. 2011a; Gilby et al. 2012) and also in a comparative analysis between grooming given and support received across both sexes by Schino (2007). Additionally, Schülke and colleagues (2010) found that preferred coalition partners were strongly bonded and not necessarily the high ranked males but frequent coalition formation predicted higher future rank in Assamese macaques (Macaca assamensis). In species with lower contest, power asymmetries may be smaller and social bond strength can have a greater influence than intrinsic fighting abilities (Ostner and Schülke submitted). These coalitions can be considered political, rank changing coalitions with mutual long-term benefits, thus males require a reliable partner to fully reap future benefits and social bonds may mediate this partner choice. Strong social bonds between partners may be important as rank changing coalitions are long-term high risk behaviours (van Schaik et al. 2006) and partner stability is required to manipulate the social status of others (Watts 2010). Additionally, "trust" between partners is required so that coalitionary activity will continue after the rank change to defend their elevated rank position 
(Ostner and Schülke submitted). Thus, coalitions under a rank changing constellation require partner choice based on previous experience to achieve long-term payoffs.

Alternatively, previous studies may have ignored an important additional factor, spatial proximity, leading to the observed correlation between affiliation and support (Puga-Gonzalez et al. 2009; Hemelrijk and Puga-Gonzalez 2012). Agent-based modelling led to predictions that individuals in groups are arranged spatially by rank, so individuals of similar rank are closest in proximity and form social relationships (Puga-Gonzalez et al. 2009; Hemelrijk and Puga-Gonzalez 2012). These same individuals are in close proximity during an aggression and therefore by default are recruited more regularly as coalitionary partners, not due to hierarchal position or social relationships per se. However, empirical studies are required to test these predictions. In a similar vein, Noë and Sluijter (1995) suggested frequent coalition formation between males may lead to false inferences about the levels of affiliation between these males. Individuals may remain in close proximity after a coalition to reduce the probability of retaliation or "stalk" their target until the opportune moment arose to attack and thus inflate time these males spent in social proximity. Here we aim to investigate the relative importance of these alternative mechanisms in predicting partner choice in coalitions of male Barbary macaques.

Barbary macaques live in multi-male, multi-female groups and males frequently form coalitions against co-resident males during the highly competitive mating season. A reproductive strategy to increase their mating success or reduce the mating success of their rivals (Kuester and Paul 1992; Widdig et al. 2000; Berghänel et al. 2010; 2011a; Bissonnette et al. 2011; Young et al. 2013a) but importantly, males also engage in rank-changing coalitions (Young et al. in revision). Wild Barbary macaque males form linear hierarchies with both infrequent solo aggression up the hierarchy and low levels of counter-aggression (Young et al. 2013a). Males form social bonds through repeated affiliative interactions prior to the mating season, males with stronger bonds are known to cooperate during the mating season (Berghänel et al. 2011a), and triadic maleinfant-male interactions (hereafter "male triadic interactions") is thought to enhance and mediate social bond strength (Paul et al. 1996; Henkel et al. 2010). The affiliative social style of male Barbary macaques, involving grooming, tolerance in social proximity and male triadic interactions (Deag and Crook 1971; Paul et al. 1996; Henkel et al. 2010; Berghänel et al. 2011a) combined with abundant male-male coalitionary aggression make the Barbary macaque an ideal species with which to examine the strength of social bonds between non-natal cohorts and partner choice during coalitionary recruitment. The available evidence does not allow to conclusively distinguish between alternative explanations for partner choice in coalitions because 1) it was not possible to rule out that the correlation between frequency of coalition 
formation and social bond strength was driven by spatial proximity, i.e. males forming coalitions with any male that was close by when they were engaged in a dyadic conflict and 2) partner choice for relative fighting power could not be disentangled from partner choice for social bond strength and 3) it is yet unknown whether the social relationships male Barbary macaques form qualify as strong social bonds.

Firstly, we look at male-male social relationships between Barbary macaques, examining if males, under natural conditions, form strong social bonds (sensu Silk 2002c; Mitani 2009; Silk et al. 2010b), i.e. differentiated, long-term, and equitable social relationships. Secondly, we examine coalitionary recruitment behaviour between males during agonistic aggression. We only consider instances with complete information about the available pool of potential coalition partners based on their proximity and attendance to the conflict. We predict that if males consider the combined fighting ability of both allies and use a simple "rule of thumb" they should always recruit the highest ranking male in the audience (maximise feasibility). We predict that if males follow attitudinal partner choice they should always solicit help from the audience member they share the strongest social bond with. We rule out that coalition pattern result from self-organization by considering only those males that are spatially close to the recruiter at the time of the recruitment. Finally, we predict that the failure to recruit a certain male, i.e. the rejection of a solicitation, may be due to social bond strength or rank position of the target and recruiter. Males may again use a simple "rule of thumb" and reject the solicitation if the rank of the target is higher than the recruiter. Alternatively males may base their decisions to reject the solicitation on the strength of their social bond to the recruiter and reject if they share a weak social bond.

\section{Methods}

Study site and subjects

Data were collected from two wild, unprovisioned groups ("Green" (Gn) and "Scarlet" (Sc)) of Barbary macaques living in a deciduous cedar and oak forest in the Middle-Atlas Mountains of Morocco (Majolo et al. 2013). The groups consisted of 7-9 adult males and 8 adult females (Gn) and 6 males and 8 females $(\mathrm{Sc})$, respectively. Data were collected on the $\mathrm{Gn}$ group from Oct. 2009 - Apr. 2011 and on the Sc group from Jul. 2010 - Apr. 2011. This study adhered to the legal requirements of Morocco, Germany and Great Britain. 


\section{Behavioural data collection and construction of the hierarchy}

Behavioural data were collected by $\mathrm{CY}$ and 5 field assistants from $0700-1900 \mathrm{~h}$. All adult males in both groups were subject to continuous focal animal observation of social and agonistic behaviour (Altmann 1974), yielding a total of 2,033 focal hours (1,676hrs Gn and 358hrs Sc). Data were collected using handheld HP IPAQ 114 series pocket PCs loaded with Pendragon Forms Version 5.1 (C Pendragon Software Cooperation, U.S.A.). Focal sampling was randomised within groups and one 40-min focal session per male was recorded per observation day so as to give an even number of focal sessions per individual. Data were further split into five 3-4-month time periods for analysis. These time periods represented distinct biological periods for the Barbary macaque groups as follows: MS09: mating season 2009 (Oct-Dec 2009), PostMS10: postmating season 2010 (Jan-Apr 2010), PreMS10: pre-mating season, coinciding with the birth season (May-Aug, 2010), MS10: mating season 2010 (Sept-Dec, 2010), and PostMS11: postmating season 2011 (Jan-Apr 2011). Data were collected on the Gn group for all periods and the Sc group for periods PreMS10, MS10 and PostMS11. The mating season was defined as the first to last observed ejaculatory copulation during either focal or ad libitum data collection (Young et al. 2013a; 2013b).

Male-male affiliative behaviour recorded during focal protocols included grooming, being in social proximity $(<1.5 \mathrm{~m})$, and male triadic interactions ("male-agonistic buffering", Deag 1980), for each behaviour, the identity of males involved as well as the duration of the activity and the initiator of the behaviour were noted.

Agonistic interactions or conflicts were defined by the occurrence of aggressive (bite, chase, slap, grab, stare, open-mouth, head-bob and charge) and/or submissive behaviours (giveground, make-room, flee, crouch submission). The dominance hierarchy was based on a total of 1,433 male-male dyadic conflicts where a clear winner and loser could be determined with no counter-aggression (Gn: MS09 - 381, PostMS10 - 535, PreMS10 - 179, MS10 - 91 and PostMS11 - 114; Sc: PreMS10 - 43, MS10 - 109 and PostMS11 - 41). Following the methods of Young et al. (2013b) a separate hierarchy was constructed for each period using corrected Normalized David's Scores (de Vries et al. 2006). The dominance hierarchies showed significant linearity with low levels of counter-aggression (4.9\% of all dyadic aggression observed), a high level of known relationships $(91.65 \% \pm 9.48$ mean $\pm S D)$ and low rates of reversals $(0 \% \pm 0$ mean $\pm S D)$ and twoway relationships $(17.62 \% \pm 14.45$ mean $\pm S D)$. 
Male social bonds: strength, stability, and equability

Male social bonds were measured using the Composite Sociality Index (hereafter "CSI"; Silk et al. 2006a; 2006b; 2010b) to examine social bond strength between male dyads in the groups. Following the methods of Silk et al. (2010b) we determined four factors of male affiliation to be highly correlated, these were duration of time in proximity ( $\min \backslash \mathrm{hr}$ dyad observed), number of friendly \neutral approaches per hour per dyad (excluding approaches resulting in agonistic behaviour), duration of male triadic interactions ( $\min \backslash \mathrm{hr}$ dyad observed) and number of male triadic interactions per hour per dyad. Male triadic interactions has been shown to be an affiliative social behaviour between Barbary macaque males previously which leads to the formation and maintenance of social bonds (Deag and Crook 1971; Paul et al. 1996; Henkel et al. 2010; Berghänel et al. 2011a). This ritualized behaviour is always bi-directional with both partners simultaneously involved in teeth-chatter and grasping of the infant (Hesler and Fischer 2007).

We also examined the grooming frequency between male dyads through-out each period. For each dyad the amount of grooming given and received was measured during focal protocols, controlling for the number of hours both individuals were observed. However, during the mating season there was a pronounced drop in grooming activity between males. For example, in the Gn group: PreMS10 we observed 88 grooming bouts between males with a mean duration of 31.6 mins, whereas during MS10 we observed only 3 bouts of male-male grooming with a mean duration of 9.7 mins. Due to this variation we did not include grooming data in the calculation of the CSI score as the infrequent grooming bouts during the mating seasons would thus highly inflate CSI scores for any dyad which did groom. Although there was fluctuations in both rates of approaches and male triadic interactions between periods this did not vary to the extreme degree of grooming bouts.

We did compare the frequency of grooming given by each dyad member for the total duration each dyad was observed outside the mating season to give an estimate of grooming reciprocity as an indicator of social bond equability (Mitani 2009; Silk et al. 2010a). These grooming frequencies for each dyad were compared for each group separately using matrix correlations as above. Male triadic interactions is the most frequent affiliative behaviour exchanged among males and is always reciprocal (Deag and Crook 1971; Paul et al. 1996; Henkel et al. 2010; Berghänel et al. 2011a) and thus, any strong bond is always equitable. 


\section{Coalitionary recruitment behaviour}

A coalition was defined using the description of Bercovitch (1988) as a simultaneous aggression by two or more males against a common target. Here we examine coalitions of two allies against one target only. Coalitionary aggression often involves rapid and complex behavioural patterns between several individuals and therefore to ensure all behaviours were observed, data of these behavioural events were recorded using handheld Kodak Zx1 HD video cameras with the observer of the aggression providing a spoken commentary of the aggression as soon as it began (for details see Young et al. 2013a). Videos and spoken records together were analysed post-hoc and either added to the focal protocol (if observed during a focal session and involved the focal animal) or ad libitum database. During each coalitionary bout the identity of the target of the coalition and the recruiter of support were noted as well as the male who was recruited to provide support (the supporter). Barbary macaque males use three behaviours to recruit an ally to a coalition, 1) "check-look", where the male faces his opponent and turns his head to the side in the direction of another male to recruit an ally ("show-look", Hesler and Fischer 2007), 2) "scream-face", where the mouth is wide open and the lips are completely retracted to show the teeth (Deag 1974; Hesler and Fischer 2007) and 3) recruitment scream (Gouzoules and Gouzoules 1995; Fischer and Hammerschmidt 2002). Only if one or more of these behaviours was directed at a specific male by the recruiter the coalition was included in the dataset for the analysis and this male was considered to have been recruited to form a coalition. All other males who were present within a $15 \mathrm{~m}$ radius of the aggression were noted and recorded as uninvolved bystanders of the aggression. All males within $15 \mathrm{~m}$, including the bystanders and the supporter, are termed "potential-supporter", the male performing recruitment behaviour is termed the "recruiter" and the recipient of coalitionary aggression is termed the "target". Male Barbary macaques are highly terrestrial and any male within $15 \mathrm{~m}$ distance would be able to join a fight within seconds (Seltmann et al. 2013; C Young, B Majolo, O Schülke \& J Ostner unpub. data).

Additionally, unsuccessful recruitment of males by the recruiter were also recorded where the recruiter used one or more of the recruitment behaviours described above directly at another male during a dyadic contest but the male rejected the invitation to support.

\section{Statistical analysis}

To investigate the stability of social bonds between males we compared each dyad's CSI score from one period to the next using row-wise matrix correlations of symmetric matrices of CSI scores for each dyad pair (Hemelrijk 1990; Hemelrijk and Ek 1991; de Vries et al. 1993; test for 
significance based on 10,000 permutations). The probability of correlation was tested by comparing Kendall's rank correlation between the matrices, performed with MATMAN ${ }^{\mathrm{TM}}$ 1.1.4 (Noldus (2003). For this analysis we included additional data on CSI scores from the PreMS11 and MS11 periods for the Gn group (Young et al. 2013a), allowing us to investigate the longevity of social bonds from Sept 2009 to Dec 2011 for the Gn group. Data collection protocol for the time period Jul - Dec 2011 differed slightly and bystander information during coalition formation data was unavailable for this time and thus, these data were not included in additional analysis. To investigate if CSI scores predicted the frequency males formed coalitions together during the mating season we adopted the same statistical procedure as above (row-wise matrix correlations) comparing the CSI scores with the frequency a dyad formed a coalition together in the mating season. For this analysis we included all coalitions observed for each dyad during the mating season.

To understand what drives a male's recruitment of a coalition partner from the audience available when a dyadic conflict occurs we adopted the following approach: firstly, a data set comprised of all coalitions in which a male recruited a supporter and two or more individuals were present as audience members was compiled (i.e. the recruiting male had a choice of at least two males to select from). For each coalition we included data for both the recruited male and the not-recruited but available male(s), i.e. bystanders. This gave a dataset with a repeated measures structure and non-independent data-points. To control for this non-independence we followed a similar approach to Kulik et al. (2012) and used a repeated random selection of all events. We used a Generalized Linear Mixed Model (GLMM; Baayen 2008) with binomial error structure and logit link function (function "Imer"; Bates et al. 2012). To establish the significance of the full model we used a likelihood ratio test, comparing the deviance with that of the null model comprising of only the intercept; the full and null model were compared using the Rfunction anova ( $R$ Development Core Team 2012). If the full model was significantly different from the null model we ran 10,000 selections of the full GLMM model to test the significance, each containing one randomly chosen data point event for each coalition, to determine the coefficients for the fixed effects. We calculated the mean of the results for each coefficient (estimate; SE; $z ; p$ ) as the result of the model.

Model 1: Is the highest ranked male or the male with the strongest social bond with the recruiter recruited? We ran a GLMM model examining if the rank of the potential-supporter or the CSI score between the recruiter and the potential-supporter influenced if the potential-supporter was recruited from the audience $(\mathrm{N}=99)$. We excluded all cases where the recruiter was the alpha male because for him the rank of the supporter may not affect the winning chances of the 
coalition and thus may not be relevant for his recruitment decision. The response variable was a binary term; whether a male from the audience (potential-supporter) was selected as a coalition partner by the recruiting male (Recruited - Yes $\backslash$ No). The predictor variables were (1) dominance rank of the potential-supporter in relation to the other members of the audience present ( $a$ binary term: highest ranked male in the audience $-\mathrm{Y} / \mathrm{N}$ ) and (2) the CSI score of the recruiter and potential-supporter. We included the identity of the potential-supporter, the identity of the recruiter and the number of audience members present as random factor. Identity of target and group were included as random factors but as these showed no influence they were removed from the analysis. Assumptions about the lack of over dispersion were respected for the analyses and we checked whether collinearity was a potential problem by using variance inflation factors (VIF) (Field 2005), using the function vif of the R-package car (Fox and Weisberg 2010) applied to a standard linear model excluding the random effects. VIFs less than ten indicate that covariation between predictors is not a problem (Bowerman and O'Connell 1990; Mayers 1990), in our analysis the range was $1.03-1.61$.

Model 2: Why does a male refuse to join a non-alpha male recruiter in a coalition? To examine if male social bond strength or rank relations influenced the potential-supporter's decision to accept or reject the recruiter's recruitment attempt we ran an additional GLMM (model 2) with binomial error structure. Whether a male chose to reject the recruiters signal for support or not was the response variable (binomial - reject recruitment - $Y \backslash N$ ), when the recruiter male was not the alpha male $(N=156)$. The response variables were $(1)$ whether the target was higher ranking than the recruiter (binomial - target higher ranked - Y/N), (2) the CSI score between the potential-supporter and the recruiter and (3) the CSI score between the potential-supporter and the target. We included the identity of the potential-supporter and the recruiter as random factors. All predictor variables were set to a mean of zero and standard deviation of one.

Where appropriate, we report mean values \pm standard deviation (SD). All statistical analysis were carried out using R 2.15.0 software (R Development Core Team 2012). The level of significance was set at $\alpha<0.05$.

\section{Results}

Male social bonds: strength, stability, and equability

In order to examine the strength of social bonds we calculated a separate score for each dyad in each group and each time period, giving a total of 177 dyadic scores. The CSI score ranges from 0 
to infinity with a mean score of 1 . High values represent a dyad with a strong social bond and those with a low value show a weak social bond. Thus, a CSI score above 1 is considered to show above average sociality compared to the other dyads in the group. In total 69 dyads showed a score of 1 or above (38.98\%), see Figure 5.1. The top $10 \%$ of dyads were above a score of 2.45 . A male had on average $2.34 \pm 1.04$ (mean \pm SD) strong bonds (partners with a CSI above 1) and $0.56 \pm 0.33$ (mean $\pm \mathrm{SD}$ ) very strong bonds (CSI score in the top $10 \%$ ).

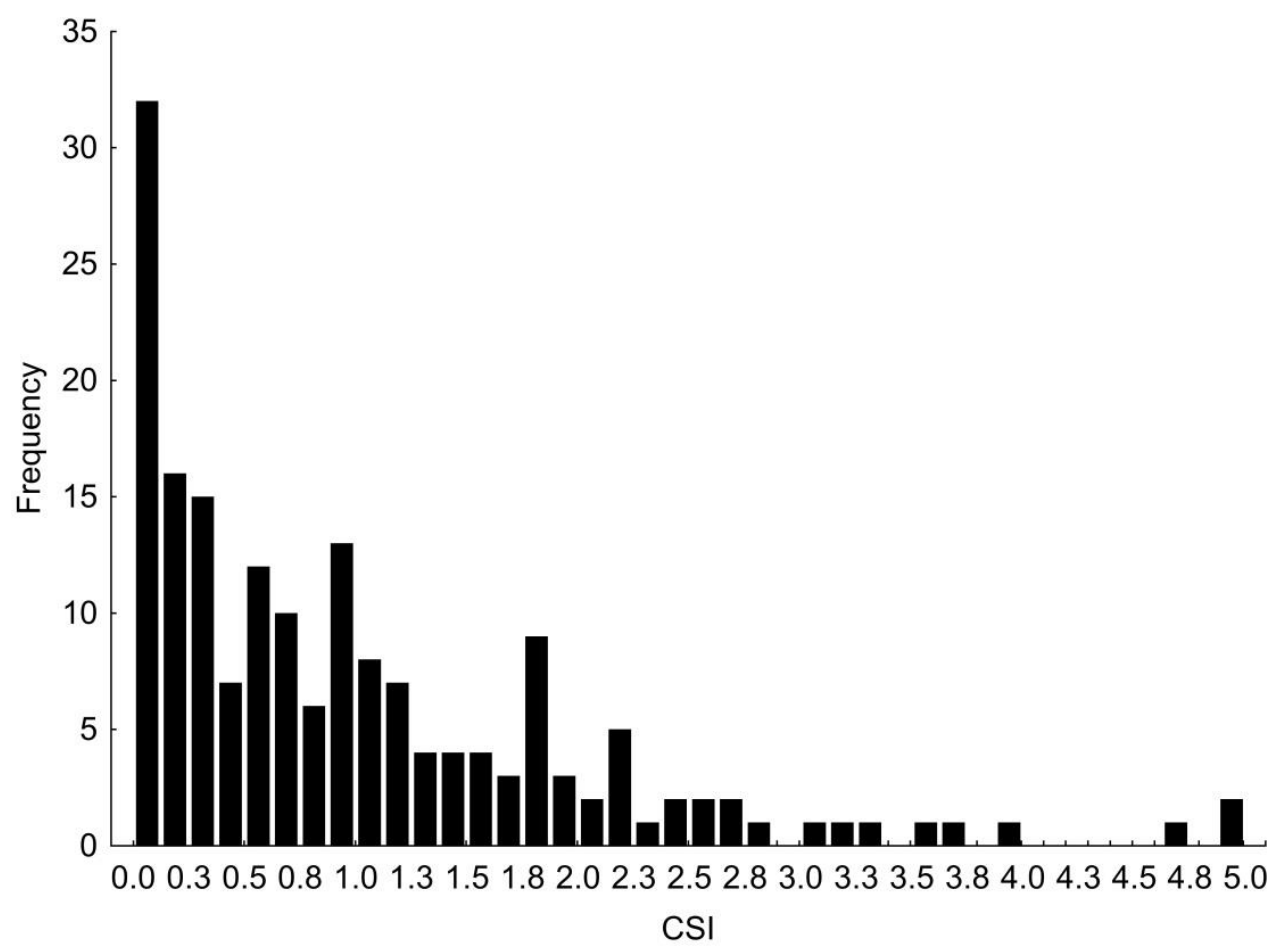

Figure 5.1: The distribution of male-male CSI scores between all dyads in the two study groups and all time periods. CSI scores of 1 and above are considered to show above average affiliation within the group. The top $10 \%$ of dyads were above a score of 2.45 .

We found the CSI scores between time periods to be significantly correlated from one period to the next for all periods across both groups, including the Gn group PreMS11-MS11 time periods (Kendal row-wise matrix correlation: mean $\tau_{\mathrm{rw}}=0.643$, range $=0.418-0.800$; mean $p=0.006$, range $=<0.001-0.033$, Table 5.1). We also compared the CSI scores of dyads in the Gn group during MS09 to CSI scores of dyads during MS11 and found dyads between these two periods separated by two years to be strongly correlated (Kendal row-wise matrix correlation: $\tau_{\mathrm{rw}}=$ $0.814, p<0.001$ ). 
Table 5.1: Kendal row-wise matrix correlation comparing the CSI scores between time periods across both groups, including the Gn group PreMS11-MS11 time periods, shown are the group, two time periods compared the Tau ${ }_{r w}$ value and $p$ value.

\begin{tabular}{lllll}
\hline Group & Time & Time & Tau & rw \\
& Period 1 & Period 2 & p value \\
\hline Gn & MS09 & PostMS10 & 0.662 & $<0.001$ \\
Gn & PostMS10 & PreMS10 & 0.800 & $<0.001$ \\
Gn & PreMS10 & MS10 & 0.586 & $<0.001$ \\
Gn & MS10 & PostMS11 & 0.601 & $<0.001$ \\
Gn & PostMS11 & PreMS11 & 0.663 & $<0.001$ \\
Gn & PreMS11 & MS11 & 0.740 & $<0.001$ \\
Sc & PreMS10 & MS10 & 0.418 & 0.011 \\
Sc & MS10 & PostMS11 & 0.571 & 0.033 \\
\hline
\end{tabular}

Social bonds were considered to be equitable due to the frequent and reciprocal affiliative behaviour of male triadic interactions (see above) which was one of the main components of our CSI score. Additionally, we also found grooming given by males to be correlated to grooming males received throughout the study (excluding the mating season) in both groups (Kendal rowwise matrix correlation: $\mathrm{Gn}: \tau_{\mathrm{rw}}=0.723, \mathrm{p}<0.001$, proportion of dyads which did not groom $=$ 0.14 ; Sc: $\tau_{\mathrm{rw}}=0.754$, mean $\mathrm{p}<0.001$, proportion of dyads which did not groom $=0.40$ ).

\section{Coalitionary recruitment behaviour}

We observed 476 male-male coalitions through-out the study period across both groups, of these 100 had more than two allies against the target and, of the remaining 376 , there were a total of 174 coalitions where 2 or more bystanders were available ( $2.64 \pm 0.83$ mean $\pm S D$, range $2-5$ ). In these coalitions the mean rank of the target was equal to $3.82 \pm 1.64$ (mean $\pm S D$ ), the recruiter was equal to $2.66 \pm 2.05$ (mean $\pm S D$ ), the supporter was equal to $2.85 \pm 1.73$ (mean \pm SD) and the not-recruited bystanders was equal to $3.82 \pm 2.04$ (mean \pm SD). Additionally, we observed 71 occasions where the potential-supporter rejected the invitation to support the recruiter and the mean rank of the target was equal to $3.65 \pm 1.72$ (mean $\pm S D$ ), the recruiter was equal to $2.89 \pm 2.04$ (mean $\pm \mathrm{SD}$ ) and the potential-supporter was equal to $2.80 \pm 1.70$ (mean \pm SD). 
Of all coalitions where two or more bystanders were present we observed $48.85 \%$ as all-down (both allies are higher ranked than the target; van Schaik et al. 2004a), 43.38\% as bridging (the target ranks in between the two allies; van Schaik et al. 2004a) and 9.77\% as all-up (two lower ranked allies against a higher ranked target; Pandit and van Schaik 2003). When examining only those where the initiator was not the alpha male, $45.45 \%$ were all-down, $37.38 \%$ were bridging and $17.17 \%$ were all-up.

We found that the CSI scores between dyads during the mating season were significantly correlated to the frequency dyads formed coalitions in both MS09 and MS10 for the Gn Group (Kendal row-wise matrix correlation: MS09: $\tau_{p w}=0.62, p<0.001 ; M S 10: \tau_{r w}=0.585, p<0.001$ ), as well as MS10 for the Sc group (Kendal row-wise matrix correlation: $\tau_{\mathrm{rw}}=0.722, p<0.001$ ) (Figure $5.2)$.

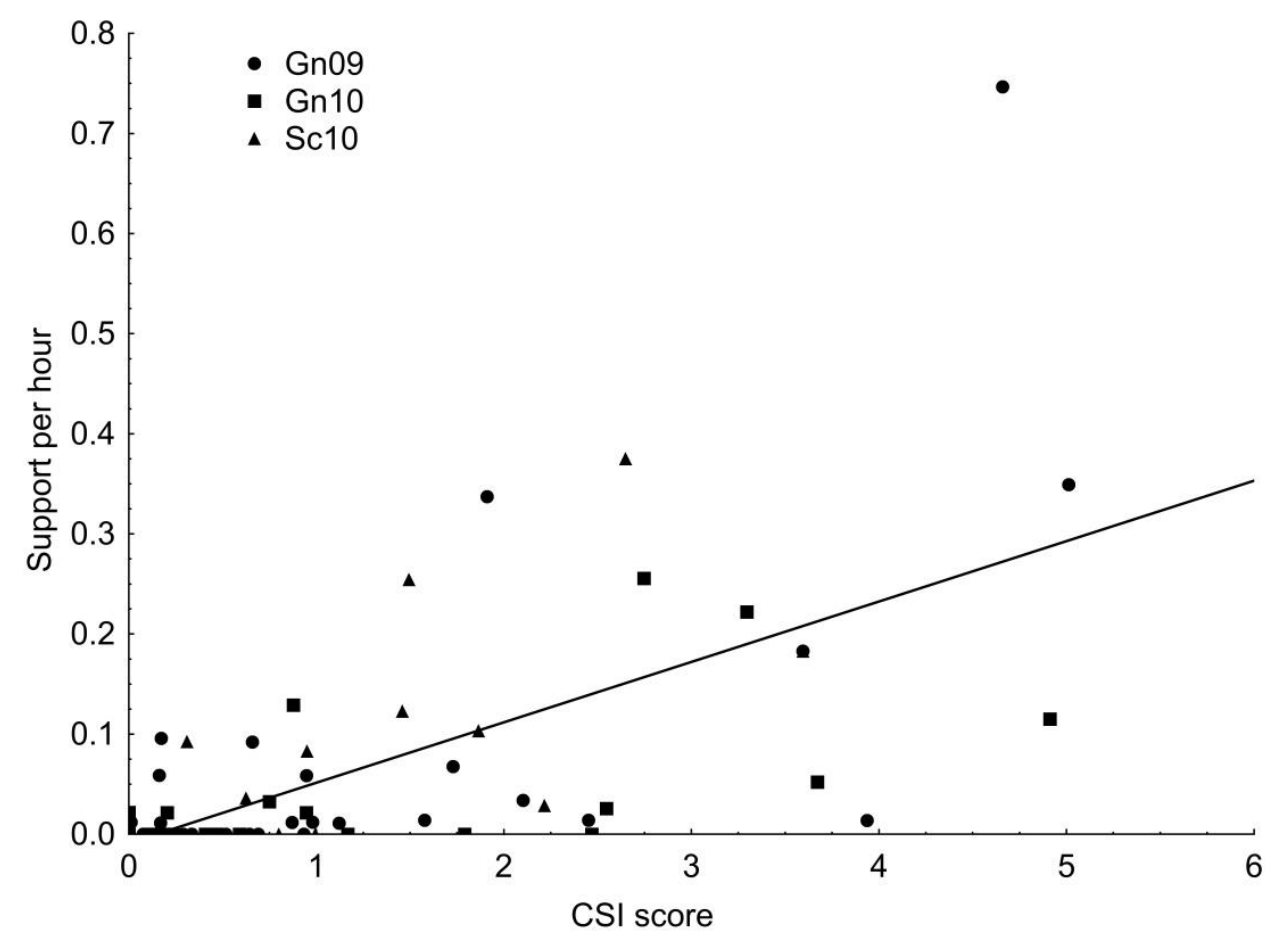

Figure 5.2: Dyadic rate of coalitions per minute for each hour the dyad was observed compared to the CSI score for that dyad. Shown are the mating seasons Gn09 (circles), Gn10 (squares) and Sc10 (triangles) $(N=71)$. 
Model 1: Is the highest ranked male or the male with the strongest social bond with the recruiter recruited?

For coalitions where the recruiter male was not the alpha male, we found that the highest ranked potential-supporter was not significantly more likely to be recruited to form a coalition than another male from the audience. However, the recruiter was significantly more likely to recruit the potential-supporter from the audience with whom he shared the strongest social bond (Table 5.2, Figure 5.3; Full vs. null model: $X^{2}=24.431, \mathrm{DF}=2, \mathrm{p}<0.001, \mathrm{~N}=99$ ).

Table 5.2: GLMM binomial regression results (mean estimates of 10,000 random selections, see methods) for the relationship between potential-supported recruited (YN) and their rank in comparison to the other audience members (was highest ranked audience member selected) and the CSI score between the recruiter and the potential-supporter, and when the recruiter was not the alpha male $(N=99)$. We controlled for the identity of the recruiter and the potential-supporter and the number of potential-supporters available.

\begin{tabular}{lcccc}
\hline Independent variable & $\begin{array}{l}\text { GLMM } \\
\text { estimate }\end{array}$ & SE & $Z$ & $P(>|Z|)$ \\
\hline Intercept & -2.185 & 0.567 & -3.851 & $<0.001$ \\
$\begin{array}{l}\text { Highest ranked audience } \\
\text { member recruited (Y/N) }\end{array}$ & 0.915 & 0.515 & 1.775 & 0.076 \\
$\begin{array}{l}\text { CSI score between } \\
\text { recruiter and potential- } \\
\text { supporter }\end{array}$ & 0.563 & 0.188 & 2.997 & 0.003 \\
\hline
\end{tabular}


Model 2: Why does a male refuse to join a non-alpha male recruiter in a coalition?

We found that both social relationships and rank relationships influenced the likelihood that a potential-supporter joined the conflict after being solicited. Firstly, we found that the potentialsupporter was more likely to refuse the recruiter's invitation to support if the target was higher ranking than the recruiter. We found that the CSI score between the target and the potentialsupporter did not influence the potential-supporters decision to reject the recruitment invitation or not, however, the potential-supporter was significantly more likely to reject the invitation to support if the social bond strength was lower between the recruiter and the potential supporter (Table 5.3, figure 5.3; full vs. null model: $X^{2}=8.848, D F=3, p=0.031$ ).

Table 5.3: GLMM binomial regression results for the relationship between a potential-supporter rejecting an invitation to support from a non-alpha recruiter $(Y / N)$ and the target being higher ranked than the recruiter, the CSI score between the potential-supporter and the recruiter and the CSI score between the potential-supporter and the target $(N=156)$

\begin{tabular}{lcccc}
\hline Independent variable & $\begin{array}{l}\text { GLMM } \\
\text { estimate }\end{array}$ & SE & $Z$ & $P(>|Z|)$ \\
\hline Intercept & -0.614 & 0.173 & -3.541 & $<0.001$ \\
$\begin{array}{l}\text { Target higher ranked than } \\
\text { recruiter }\end{array}$ & -0.385 & 0.178 & -2.157 & 0.031 \\
$\begin{array}{l}\text { CSI score between } \\
\text { recruiter and potential- }\end{array}$ & -0.399 & 0.180 & -2.223 & 0.026 \\
$\begin{array}{l}\text { supporter } \\
\text { CSI score between target } \\
\text { and potential-supporter }\end{array}$ & -0.059 & 0.183 & -0.324 & 0.746 \\
\hline
\end{tabular}




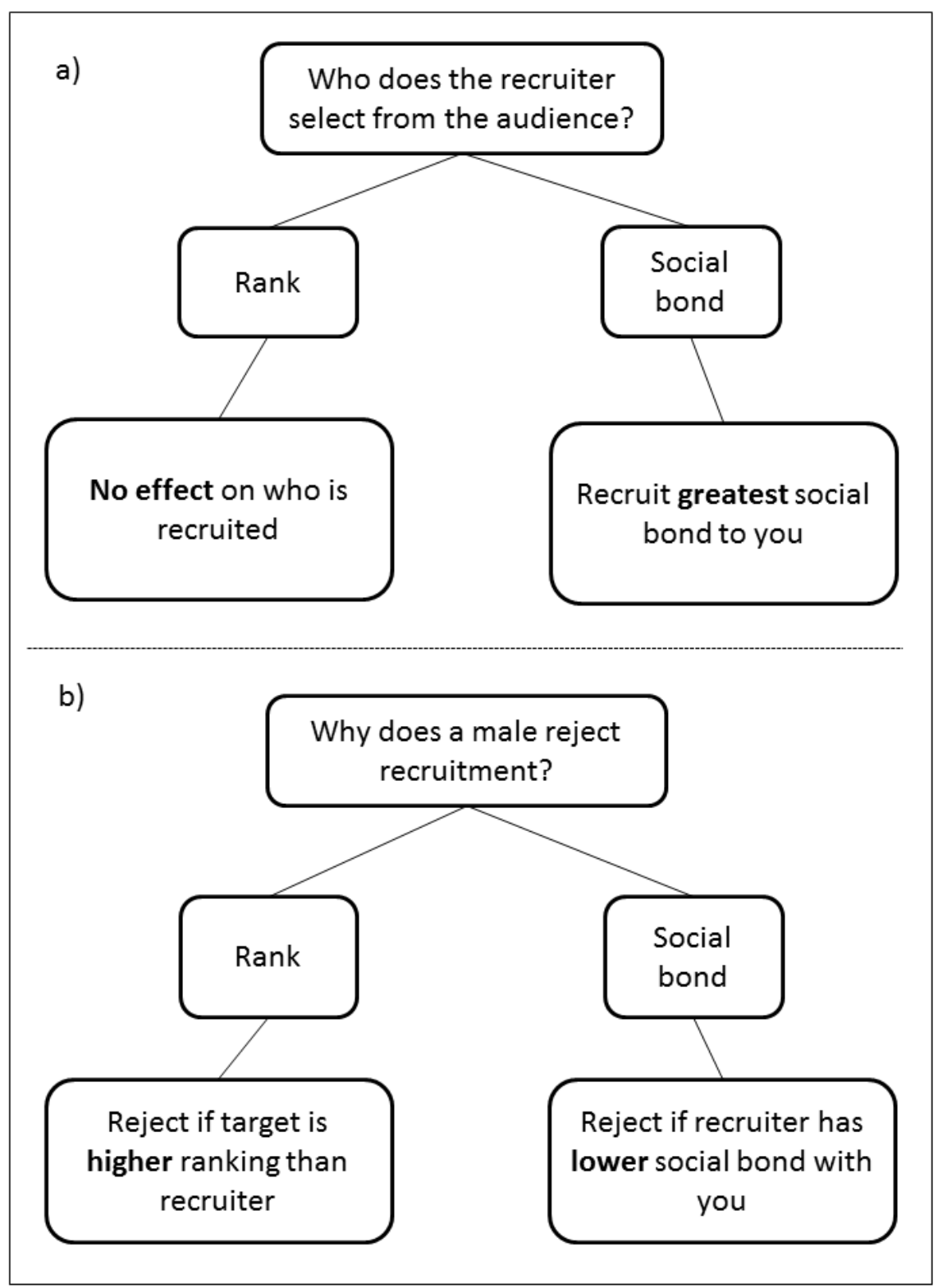

Figure 5.3: Summary of the results of a) the recruiter male's recruitment decisions in relation to the social bond strength between the recruiter and potential-supporter and if the highest ranked male from the audience was recruited and b) a males decision to reject a recruitment invitation from another group male in relation to social bond strength and if the recruiter male or the target male was higher ranking. 


\section{Discussion}

Wild Barbary macaque males of our study formed stable, differentiated social bonds, similar to that of female yellow (Papio cynocephalus, Silk et al. 2006a; 2006b) and chacma baboons ( $P$. ursinus, Silk et al. 2010a), male chimpanzees (Mitani 2009) and Assamese macaques (Schülke et al. 2010). In a species with a short mating season, relationships may be expected to break down during the mating season. However, social bonds were correlated over two years continuously including three consecutive mating seasons. Although under-free ranging conditions short-term social bonds between Barbary macaque males have been observed (Henkel et al. 2010; Berghänel et al. 2011a), we find compelling evidence of long-term strong male bonds in wild populations where migration is unrestricted and neighbouring groups are numerous (C Young, B Majolo, O Schülke \& J Ostner unpub. data), thus bond formation cannot be considered a possible remnant of free-ranging conditions. Grooming was not distributed evenly between all dyads, in fact some dyads were not observed to groom. Although the correlation between grooming given and received could be considered an artefact of the proportion of zero grooming dyads in the Sc group, this is unlikely to be the case in the Gn group few dyads showing no grooming and likely to be a true representation of the grooming differentiation between dyads. The formation and maintenance of strong social bonds can be temporally demanding (Dunbar 1992; Dunbar et al. 2009) and in Barbary macaques, where males use triadic male-infant interactions as a social mediator, can also be physiologically costly (Paul et al. 1996; Henkel et al. 2010). Therefore, this potentially costly behaviour of bond formation and maintenance should provide benefits, which may come in the form of coalition formation. As such, we further investigated the function of social bonds in male coalitionary recruitment behaviour and found that recruiting males are more likely to recruit a male from the audience with the greatest social bond to themselves. From the perspective of the male being recruited we found that non-alpha males were more likely to reject an invitation to support if the potential-supporter was lower ranked than the target or if the strength of the social bond between the recruiter and the potential-supporter was low.

Coalitionary recruitment, in our study, may be based on more than the rank of the bystanders available; in fact, we found that the highest ranking potential-supporter was not selected from the audience. Bissonnette and colleagues (2009a) suggest Barbary macaques under free-ranging conditions maximise the feasibility of the coalition by recruiting the highest ranked male available. However, the vast majority of coalitions were all-up (Pandit and van Schaik 2003) where the rank and hence fighting abilities of the partner may play a much greater role (van Schaik et al. 2006). Unfortunately, no data on social relationships between males were available 
during this study. A further study on the same group (Berghänel et al. 2011a) found more affiliated dyads before the mating season were more frequent coalition partners during the mating season, so we cannot rule out a possible influence of male social bonds. A further study on free-ranging Barbary macaques found males to be more likely to support their kin than nonkin (Widdig et al. 2000) highlighting that more than hierarchal position is considered when supporting in coalitionary aggressions. In our study, males were more likely to reject to support the recruiter, if the target was higher ranked than the recruiter, and if the social bond strength to the recruiter was weak. Similarly, hyenas (Crocuta crocuta) were shown to support higher ranked individuals during dyadic contests (Engh et al. 2005). It may be that individuals are less likely to join a coalition against a higher ranked target for fear of future "punishment" (CluttonBrock and Parker 1995; Clutton-Brock 2009a).

Partner recruitment during coalitionary aggression has been suggested to be a by-product of spatial proximity within social groups (Puga-Gonzalez et al. 2009; Hemelrijk and Puga-Gonzalez 2012). However, we found males to recruit coalition partners based on social bond strength even after controlling for those individuals available when the aggression occurred. Similarly, Noë and Sluijter (1995) suggest that estimates of male association may be inflated by frequent coalitionary aggression between partners, as coalitionary behaviour leads them to associate more frequently and spend longer periods in close proximity. This may be the case for species, such as yellow baboons, where male affiliation is very rare, reproductive skew is high, high ranked males consort females, coalition formation may be more opportunistic and partner choice may occur on a short-term basis dependant on the current needs of the participants (Noë 1992; 1994; Noë and Völkl 2013). In species with large power asymmetries and infrequent male affiliation, partner choice for coalitions may be based on fighting abilities with only a handful of males able to provide the intrinsic power to defeat a high ranked rival (Noë 1992). Due to high skew and frequent consorts by high ranked males, opportunistic levelling coalitions are a viable option for lower ranked individuals (coalitions under a sexual context; Pandit and van Schaik 2003) to break up consorts and gain access to consorted females (Bercovitch 1988; Noë and Sluijter 1990). These opportunistic levelling coalitions, when reproductive skew is high, may not require long-term partner choice. These coalitions may be mediated by short-term attitudinal partner choice based on both positive experience with pervious partners and the identity of the target (Noë 1992; 1994; Noë and Völkl 2013). Males can gain short-term benefits through levelling coalitions and the development of long-term social bonds may not be necessary under high contest conditions. 
Coalitionary activity does not occur only over direct access to females, males can form coalitions leading to changes in the dominance hierarchy (rank changing coalitions; van Schaik et al. 2004a; 2006). Rank change due to coalitionary aggression tends to require many aggressive events so males may still recruit partners based on attitudinal partner choice but over a longer time frame based on both previous experience of coalitionary activity and affiliative behaviour (Schülke et al. 2010; Ostner and Schülke submitted). Cooperating with conspecifics during aggressive encounters is high risk: if one ally were to defect and leave the coalition it would leave their partner in a vulnerable position with an increased risk of injury (van Schaik et al. 2006). Males can use frequent affiliation to strengthen social bonds and recruit these partners during coalitionary aggression (Silk 1992; Perry et al. 2004; Perry 2012). Animals sharing a strong social bond are less likely to defect and a coalition between a dyad without defection would enhance the "trust" in the relationship. This may further enhance the strength of the bond whereas if defection were to occur a negative association to that partner would be made (van Schaik et al. 2006; Schino and Aureli 2009).

Male chimpanzees trade grooming for coalitionary support (Watts 2002) and males who are central to the male coalitionary network (determined through social network analysis) are more likely to rise in rank and increase their reproductive success (Gilby et al. 2012). Additionally, strong social bonds in Assamese macaque males led individuals to frequently form coalitions together and gaining high future rank positions, ultimately leading to an increased future reproductive success (Schülke et al. 2010). Males without strong bonds fell in rank or remained low ranking. Maintaining a high rank position, once it is acquired, may also require coalitionary support. Thus, strong social bonds may aid the longevity of support between partners even when high rank status is achieved, through conservative coalitions (van Schaik et al. 2006). Barbary macaques frequently form both levelling and rank changing coalitions (Kuester and Paul 1992; Widdig et al. 2000; Berghänel et al. 2010; Bissonnette et al. 2011; Young et al. 2013a; Young et al. in revision). Currently it is unknown if coalition formation in wild groups has a direct benefit of increased paternal reproductive success but males do increase their mating success through coalition formation (Young et al. 2013a). In both chimpanzees (Gilby et al. 2012) and Assamese macaques (Schülke et al. 2010) attitudinal partner choice guides coalition formation and in turn affects both future rank and paternity success. Thus, Barbary macaque males may form strong, stable, equitable social bonds similar to those in female baboons (Silk et al. 2006a; 2006b; 2010a), male chimpanzees (Mitani 2009) and macaques (Schülke et al. 2010). Males with long-term, strongly bonded partners may gain both short-term benefits, reducing the risk of injury and partner defection, and long-term benefits by increasing reproductive success. 
Our study builds on previous knowledge of male affiliation and coalitionary activity, helping to fill in some of the gaps and open questions of previous studies. By undertaking a longer term study under natural conditions we build on the previous findings of Berghänel et al. (2011) finding males do form long-term, strong social bonds. Additionally, adding to on the Bissonnette et al. (2009) study, we found that males base partner choice on more than hierarchal position when recruiting their coalition partner. Specifically, males may use social bond strength and previous experience with partners when making recruiting decision, although, comparisons to social bond strength was not possible with the Bissonnette at al. study. Under certain conditions, such as all-up coalition formation, partner choice may be more opportunistic or rank driven to maximise the feasibility of the coalition. Thus, under these circumstances rank position of supporters as well as bond strength may play a larger role, as in the Bissonnette et al. (2009) study where all-up coalitions were frequent. Our results add to the growing body of evidence that social bonds are an important functional tool in guiding partner choice, ultimately leading to fitness benefits, even for the dispersing sex. Strong bonds may have evolved together with rankchanging coalitions that serve to manipulate others' social status which is now echoed in the human literature on friendship (DeScioli and Kurzban 2009). Although males appear to use attitudinal partner choice recruit the bystander with the strongest social bond to them this does not rule out the possibility that they still use simple rules when recruiting; "select the male with the greatest social bond" is still cognitively simple. However, males do need to maintain strong bonds with their regular coalition partners and prevent bonds developing between other individuals in the group, the recognition of third party social and rank relationships between other group members remains to be answered. Furthermore, a comparative analysis across species and taxa is necessary to examine the relationship between sociality and coalition formation and to determine if these two factors have evolved in line with increased brain size, as suggested by the social brain hypothesis (Dunbar 1995; 1998). 


\section{Acknowledgements}

The authors are grateful to Professor Mohamed Qarro (Ecole Nationale Forestière d'Ingénieurs, Morocco) for his support in the field and the Haut Commissariat aux Eaux et Forêts et à la Lutte Contre la Désertification of Morocco for research permission. We would also like to thank Michael Madole, Dave Thomas, Sofia Santos, Maria Thunström, Tom Smith, Josephine Msindai and Sabine Hähndel for assistance in the field. We thank Laëtitia Maréchal, Sally MacDonald and Cédric Girard-Buttoz for helpful comments, Christof Neumann for statistical advise and Andreas Berghänel and Annie Bissonnette for fruitful discussions. Financial support was provided by the Max Planck Society, the Christian Vogel Fonds and Georg-August University Göttingen through funds from the German Initiative of Excellence. 


\section{Chapter 6}

\section{General Discussion}

For cooperation to evolve in group living species the benefits of acting together must be high enough to outweigh the costs (Hamilton 1964a; 1964b; Trivers 1971). In many mammalian species individuals have been shown to cooperate and increase their reproductive success, with cooperation being more prevalent in the philopatric sex (reviewed by Silk 2002a; Silk 2007) where it can lead to inclusive fitness benefits. Males are generally the dispersing mammalian sex, but cooperation between males has been observed under many circumstances including hunting (Blundell et al. 2004), protection from predators (Waterman 1997), grooming exchange (Kutsukake and Clutton-Brock 2010), defence of females (Packer and Pusey 1982; Fedigan and Jack 2004; Port et al. 2010; 2012), territory defence (Caro and Collins 1987) and male-male coalition formation (reviewed by Olson and Blumstein 2009; Smith et al. 2010), to name but a few examples. In this thesis I focused on male cooperation in the form of coalitionary aggression. Males can cooperate opportunistically in the short-term and gain immediate benefits (levelling coalitions; Pandit and van Schaik 2003) or to increase rank and gain future reproductive payoffs (rank-changing coalitions; van Schaik et al. 2004a; 2006). These coalition types are predicted to occur at different levels of within group contest potential. I found that male macaques form long-term social bonds which may have an adaptive benefit by facilitating regular, reliable partner choice for coalition formation. I discuss the possible benefits of regular coalitionary partners in terms of long-term mating/reproductive strategies below.

In this general discussion I will firstly briefly summarise the main findings of my thesis (section 6.1). I then discuss the need for empirical estimates of contest potential (section 6.2) and the influence of contest competition on mating skew (section 6.3). I discuss how different levels of male contest potential affect male cooperation in the form of coalition formation within groups, at very high/low levels of contest (section 6.3), at high (section 6.4) and mid-low levels of contest (section 6.5). Coalition formation in non-primate groups are then discussed (section 6.6). I suggest possible mechanisms for the evolution of coalitionary cooperation in primate groups (section 6.7). Finally I provide a general outlook (section 6.8). 


\subsection{Summary of results}

By investigating the complete chain of evidence from male contest competition to mating success and cooperation via male social bonding, this study unites several previously separate pieces of research into one comprehensive picture. As stated above male coalitionary cooperation is thought to hinge on the level of within group contest competition (van Hooff and van Schaik 1992; van Schaik et al. 2004a). Thus, I first investigated the extent to which wild Barbary macaque males can ascertain information about female reproductive state (Chapter 2). I found that within conception cycles ovulation was most likely to occur during the maximum swelling period. However, male mating behaviour was further concentrated around the fertile phase implying that males infer information from more than swelling size alone. Male mating frequency increased in line with female socio-sexual behaviour. Most strikingly my results showed that males invested equally in mating during fertile and non-fertile, i.e. post-conception, maximum swelling phases. Although previous studies have investigated male mating behaviour in free-ranging Barbary macaques (Kuester and Paul 1984; Möhle et al. 2005; Brauch et al. 2007; Heistermann et al. 2008; Bissonnette et al. 2011; Pfefferle et al. 2011) this is the first study to have investigated the behavioural significance of post-conception swellings for male reproductive behaviour in the species. Whether post-conception swellings were merely a result of changes in hormone concentrations during pregnancy or part of a female reproductive strategy remains elusive.

By understanding what males can infer about female reproductive state I was then able determine group specific estimates of male mating skew as predicted by the Priority of Access model (PoA model; Altmann 1962). Consecutively across three mating seasons I examined wild Barbary macaque mating skew and compared it to the predicted skew (Chapter 3 ). I found that mating was skewed up the hierarchy, i.e. that the higher a male ranked in the dominance hierarchy the more matings he had. High ranked males did not gain as high mating success as predicted by the PoA model suggesting that female receptive synchrony was an effective strategy to reduce male monopolisation. Females initiated sexual encounters frequently with mid-ranked males increasing their mating success and these males were frequent recipients of aggression via bridging coalitions. Males who cooperated through coalitionary aggression were also found to increase their mating success. I concluded that high ranked males utilize bridging coalitions to counteract the effects of female behaviour and reduce mating being concentrated in one main rival. High ranked males may not gain direct access to females via coalition formation but as they cannot control mating access it may be better to disrupt future mating 
opportunities of co-residents and prevent females from concentrating mating in a particular mid-ranking rival.

I examined the Pandit/van Schaik model (PvS model; Pandit and van Schaik 2003; van Schaik et al. 2004a; 2006) for within group coalition formation using group specific empirical measures of male contest competition (Chapter 4). Although I found support for several predictions of the model it appears that the costs and benefits may have been underestimated in the model and some of the predictions did not concur with the data. Particularly it may be that the model assumes immediate benefits and costs to coalition partners but there may be long-term benefits to cooperating which can be mediated by differentiated social bonds (a factor not examined in the PvS model). These longer-term benefits may explain the abundance of rank-changing coalitions at low contest potential not predicted by the model in Assamese macaques. Taking this a step further I then investigated male social bond strength and partner choice during recruitment for coalitionary aggression in Barbary macaques (Chapter 5).

I found that male Barbary macaques formed strong, stable and equitable social bonds similar to other primate males or females (Silk et al. 2006a; 2006b; 2010; Mitani 2009; Schülke et al. 2010), and these bonds were maintained through periods of intense male competition (mating seasons). The formation and maintenance of strong social bonds can be costly, both temporally (Dunbar 1991) and physiologically (Henkel et al. 2010). However, I found a benefit to diminish the potential costs in the form of stable partner choice in coalitionary aggression. I investigated the function of social bonds in male coalitionary recruitment behaviour and found that recruiting males selected the male from the audience with whom they shared the strongest social bond. Social bonds between males may mediate long-term partner preferences for rank-changing coalitions and males may recruit partners based on their social bond strength so as to gain both short-term benefits, reducing the risk of injury and partner defection, and potentially long-term benefits by increasing reproductive success. Thus, mid-low contest can lead to the development of strong social bonds which act to foster reliable partner choice for cooperation through coalitionary aggression.

\subsection{Estimating contest potential}

Males and females face a continual arms-race in terms of competing reproductive strategies (Trivers 1972). In many species of primate, females extend receptivity beyond the period of fertility (Hrdy and Whitten 1987). Thus, the extent to which primate males can determine female fertility varies greatly across species (for example Heistermann et al. 2001; Deschner et al. 2004; Engelhardt et al. 2007; Fürtbauer et al. 2011a; 2011b). In some species the female fertile period 
is clear whereas in others females can conceal ovulation or use reproductive mechanisms/strategies to mate with males outside the period of fertility (reviewed in Chapter 3). If males cannot infer female reproductive state then estimates of contest potential for this species must be adjusted. Highlighted in this thesis (Chapter 3 ) where Barbary macaque females can extend receptivity further with an additional sexual swelling during pregnancy which the males cannot distinguish from swellings when ovulation occurs. Males invest equally in all swelling phases which can be both temporally and energetically costly and highlights an important, perhaps overlooked, aspect of the examination of reproductive strategies. Models predicting both cooperation (PvS model; Pandit and van Schaik 2003; van Schaik et al. 2004a; 2006) and mating/reproductive skew (PoA model; Altmann 1962) hinge on accurate estimates of contest potential. So species or group specific measures of the information males can infer about female reproductive state are essential. Thus, a bottom-up approach firstly investigating male knowledge of female reproductive state can provide a solid foundation with which to examine male reproductive strategies.

\subsection{Male contest and mating skew}

A call for species specific measures of male knowledge of female reproductive state is emphasised by studies examining the PoA model, as discussed by several authors (Alberts et al. 2003; Wroblewski et al. 2009; Henzi et al. 2010, this study Chapter 3). The model's predictions are based on both female receptive synchrony and male ordinal rank position (Altmann 1962) and it is crucial to use accurate estimates of female reproductive synchrony based on information males can infer. The predictions of the PoA model can provide an empirical estimate of male contest potential within primate groups. Cooperation is predicted to evolve at different levels of contest competition so gaining empirical estimates is pertinent to the study of the evolution of cooperation in primate species (Pandit and van Schaik 2003; van Schaik et al. 2004a; 2006). Indeed the mixed support for the PoA model may in part be due to the estimates of female receptive synchrony used (Alberts et al. 2003; Wroblewski et al. 2009; Henzi et al. 2010). Although several post-hoc measures of within group contest have been suggested, including alpha male paternity success (van Schaik et al. 2004a; 2006), these measures rely on the males being able to ascertain accurate information about female reproductive state. If males can infer when the fertile phase occurs then competition will be concentrated around the fertile phase and the alpha male should be able to monopolise access to females (depending on receptive synchrony levels). However, males cannot infer when the fertile phase occurs then competition may not be concentrated solely around the fertile phase and thus, paternity success may not encapsulate the mating effort and contest within the group post-hoc. Many factors can act to 
lower alpha male paternity including the number of males, number of females, female mate choice, the energetic costs of mate-guarding and the information males can infer about female reproductive state, although this list is by no means exhaustive (reviewed by Alberts 2012). The PoA model predictions allow a measure of contest potential based solely on the expected mating/reproductive skew depending on the number of synchronously receptive females (Chapter 3 ) thus, before these additional factors can affect alpha male paternity.

\subsection{Contest potential and cooperation}

Living in multi-male, multi-female relatively stable groups carries increased costs for males in terms of a greater number of competitors for access to females (Kutsukake and Nunn 2006; Schülke and Ostner 2012). Contest potential within groups ranges from very high to very low (van Schaik et al. 2004a; 2006). At very high contest one would expect the alpha male to gain complete monopoly of access to females and the number of males in the group may be limited as reproductive benefits may diminish rapidly down the hierarchy. Cooperation between males may be limited on two fronts; firstly, variability in male group number with many groups containing few males would restrict the opportunity to regularly form coalitions (Henzi and Barrett 1999; van Schaik et al. 2006). For example, chacma baboon males show very high skew to the alpha male and coalition formation appears to be absent (Henzi and Barrett 1999; 2003; Henzi et al. 2010). However, male group size is variable and often very low and thus, over evolutionary time frequent opportunities for coalitionary aggression may not have manifested (Henzi and Barrett 2003). Secondly, there may be large power differentials between individuals so that one male can gain full monopoly of access to receptive females. Therefore, cooperation is unlikely to evolve as the power asymmetry will be too high between males for coalitions to be viable. For example, in mandrills males have not been observed to form coalitions (Setchell and Wickings 2005) and both the observed and the PoA predicted mating success is highly skewed towards the alpha male (Setchell et al. 2005). Due to extreme sexual dimorphism in the species (Plavcan and van Schaik 1992) even sexually mature males need to reach large body size before they can compete with the alpha male further enhancing alpha monopoly.

Conversely, if contest potential is extremely low then scramble competition is likely to develop and all males can gain access to females and thus, cooperation provides costs but no benefits. For example, in muriquis (Brachyteles hypoxanthus) females and males are co-dominant; males do not form a discernible hierarchy and are unable to determine female reproductive state (Strier 1994; Strier et al. 2011). Males do form affiliative relationships within the group but these are undifferentiated and no coalitionary activity between males has been observed. This 
illustrates that more than affiliation alone is required for cooperation to evolve and there must be a benefit to the individuals to offset the costs and drive the evolution of cooperation.

\subsection{High within-group contest and cooperation}

As contest competition begins to decrease (from very high) the opportunity for the evolution of cooperation between males may become feasible. At high contest the top ranked males can still monopolise access to receptive females. In these species, such as yellow baboons, there are still differences in size and fighting abilities between males but these are not as pronounced as with very high contest (Bercovitch 1988; Noë and Sluijter 1990; Noë 1992; 1994). Thus, certain combinations of males (usually of mid-rank) can team up and form coalitions to break up consortships. These coalitions are most likely to be opportunistic depending on the identity of the consorting male and are usually all-up coalitions as dominant males consort frequently (van Schaik et al. 2004a; 2006). When recruiting a partner, males must weigh-up the available allies and the target to recruit a partner who will provide enough combined intrinsic fighting ability to defeat the target.

The undecided nature of the payoff outcome can make the opportunistic coalitions attractive for both partners (Noë 1990). Although an uneven payoff accrues, either male could gain access to the female and not necessarily the highest ranked ally, for example, in baboons (Bercovitch 1988; Noë 1992) and macaques (Bissonnette et al. 2009a; 2011). Both benefit by breaking up the consortship and simultaneously reducing the future mating opportunities of the consorting male and increasing the probability of future mating opportunities for the coalition partners. Barbary macaque coalition partners were shown to chase consorting targets up trees, isolating them and limiting their mating access, an effective strategy in a mainly terrestrial species (Kuester and Paul 1992; Bissonnette et al. 2011). Thus, short-term cooperation between males can lead to immediate payoffs for both parties breaking up future mating opportunities for the consorting male. Similarly, in savannah baboons (Alberts et al. 2003) a long-term study found that the PoA model predicted a high male mating skew and thus high contest competition. The empirical data varied greatly across years and groups to the PoA model's predictions. This variation was attributed to differing age demographics leading to variable fighting abilities between males, the number of males present, the energetic demands of extended mate-guarding and levelling coalitions by lower ranked males (Alberts et al. 2003). Where moderately high skew and relatively large power differentials occur coalitionary activity can evolve which requires shortterm cooperation and a relatively quick return of benefits, although not necessarily immediate direct access to females (Pandit and van Schaik 2003). 


\subsection{Mid-low within-group contest and cooperation}

At mid-low contest potential the capacity of the alpha male to control mating access to females weans further due to increasing female receptive synchrony. However, despite not being able to fully control mating access high ranked males can gain a somewhat higher proportion of mating/reproductive success (Schülke et al. 2010; this study Chapter 4). Power asymmetries generally decease with declining contest and attainment of higher rank positions through coalition formation becomes a more viable option. This may be a longer term form of cooperation than that seen during high contest potential as individuals may require repeated interactions once they have attained higher rank position to defend their higher status, see below (van Schaik et al. 2006; Ostner and Schülke submitted).

The mechanism of attitudinal partner choice may drive long-term cooperation as individuals recruit partners due to their previous experiences with them (Noë and Völkl 2013; Ostner and Schülke submitted) and may be mediated by social bonding between males. Strong social bonds between individuals may have developed as a mechanism for individuals to judge the reliability or reputation of other group members. Individuals may use the willingness to affiliate as an advertisement for willingness to cooperate in the future (Berghänel et al. 2011a). A willingness to invest (e.g. grooming or male triadic interactions) may transfer into support during aggression. Barbary macaque males did not select coalition partners via "public reputation" to cooperate and may have used "private reputation" or individual private experience when selecting coalition partners (Berghänel et al. 2011a), further suggesting attitudinal partner choice.

Rank-changing coalitions carry high immediate costs to the participants as higher ranked targets can show counter-aggression (van Schaik et al. 2006) and sexual selection has equipped males with extensive weaponry (Plavcan and van Schaik 1997; Setchell 2003; Plavcan 2004; Emlen 2008). Recruitment of a coalitionary partner should therefore consider their previous experience with a partner because if the partner were to defect then they would be left in a vulnerable position possibly leading to serious injury (Ostner and Schülke submitted). A rank reversal most likely occurs after one specific coalitionary event but may require several attempts to achieve this goal. Furthermore, fighting abilities remain the same despite elevated rank position, so partners should continue to form coalitions to defend and cement the newly acquired higher rank position (van Schaik et al. 2006; Ostner and Schülke submitted). Additionally, a regular coalitionary partner can act to increase a males intrinsic power even in the absence of the partner and function to intimidate opponents and reduce the probability of aggression from higher rank individuals (Berghänel et al. 2011b). 
Bridging coalitions are predicted to occur between kin as only by assisting a relative can they remain profitable for the high ranked partner (van Schaik et al. 2004a; 2006). Where rankchanging coalitions occur between bonded partners, all-up and bridging coalitions can be variable depending of the ranks of the two bonded allies if both males can gain mutual benefits. As one or both individuals climb in rank, coalition constellations occurring would change and if both males attain top ranks (alpha/beta) then all-down coalitions would be frequent to protect the new status. Alternatively, if only one male climbs up the hierarchy defensive bridging coalitions between the two partners may occur or these may be bridging rank-changing as the partners attempt to increase the lower ranked male's position. Hence, social bond strength may explain the frequent occurrence of bridging coalitions in species with male dispersal (Schülke et al. 2010; this study Chapter 5).

Male Assamese macaques show low contest competition, although the alpha male still gains the greatest share of matings (Ostner et al. 2011) and thus, it pays to be high ranking. Males who form strong social bonds more frequently cooperated through coalition formation and gain a higher future rank position. Strongly bonded partners were not necessarily related and were not predicted by the current rank position of the partners. Males without strong bonds tend to remain at low rank or in the future decrease in rank (Schülke et al. 2010). Additionally, in both bonnet macaques (Macaca radiata) and white-faced capuchins (Cebus capucinus) males with stronger social bonds are recruited more frequently in coalitionary aggression (Silk 1992; Perry et al. 2004; Perry 2012). Similarly, in chimpanzees males affiliate frequently and can form longterm social bonds, often with non-kin (Langergraber et al. 2007; Mitani 2009). Furthermore, chimpanzee males who groom frequently form coalitions frequently (Watts 2002) and males who provided coalitionary support to the alpha gained increased mating opportunities in the presence of the alpha than males who did not (Duffy et al. 2007). Furthermore, wild Barbary macaque males form strong social bonds between group mates and these bonded males were more likely to be recruited as coalitionary partners (Chapter 5). Although paternity data is lacking, mating was skewed up the hierarchy. Thus, cooperation through rank-changing coalitions to improve status may provide fitness benefits and require a dependable partner which may be mediated by social bond strength (Chapter 5). These studies highlight the important role strong, differentiated social bonds may play in male cooperation in primate societies.

Social bonding between males, however, is not a prerequisite for rank-changing coalitionary activity as has recently been shown in rhesus macaques where males show little affiliative behaviour but all-up rank-changing coalitions were observed (Higham and Maestripieri 2010). 
Although these coalitions involved several allies and may be described more like a series of "gang-attacks" to over-throw the high ranked males. Indirect benefits can accrue through support of the alpha male, such as access to feeding sites in Tibetan macaques (Macaca thibetana huangshanensis) (Berman et al. 2007). The formation of coalitions by the alpha male served to reinforce his status and reduce opportunity for coalitions between lower ranked males. Generally, the three highest ranked males showed high levels of support and tolerance to each other and gained 93\% of all matings (alpha 64\%). With all individuals below rank 3 having to share $7 \%$ of mating opportunities it would pay to be high ranked. A stable hierarchy was maintained through all-down defensive coalitions as a lower ranked male rising to alpha position would be detrimental to the mating success of each of the top three males. Coalitionary support may act in a similar manner to that expressed in Assamese macaques succeeding a rank-change, as the high ranked males use coalitions to fend off challenges from lower ranked males and maintaining their high status. There was no correlation between grooming given and agonistic support received between males but as there was no direct measure of bond strength it may be possible social bonding plays a role in partner choice but this requires further study.

\subsection{Non-primate mammalian coalitions}

Out with non-human primates, the expression of coalitions between males in mammalian taxa is rare (reviewed by Olson and Blumstein 2009; Smith et al. 2010). However, the formation of coalitions in non-primate mammalian males does still merit discussion as many of the patterns of coalition formation observed mirror those seen in primate societies (Harcourt 1992). Firstly, several social carnivores have been shown to cooperate in group defence of females, including lions (Panthera leo) (Packer and Pusey 1982; Packer et al. 1988; Grinnell et al. 1995), slender mongooses (Herpestes sanguineus) (Waser et al. 1994) and cheetahs (Acinonyx jubatus) (Caro and Collins 1987). These between group coalitions have similar behavioural hallmarks of between group coalitionary defence of females in primate groups. Several ungulates which live in loose aggregations during the breeding season have also been shown to intervene in on-going dyadic contests to increase their reproductive success, such as red deer (Dama dama) (Jennings et al. 2009; 2011), Camargue horses (Equus caballus) (Feh 1999) and semi-free ranging plains zebra (Equus burchelli) (Schilder 1990). These loose aggregations are very different from the relatively stable groups of multi-male, multi-female primate groups.

Cases of within group cooperation in the form of coalitionary aggression in non-primate mammalian species are scant (Harcourt 1992). High ranked wild dog (Lycaon pictus) males were found to form coalitions against mid-ranked rivals to reinforce their hierarchal position (de Villiers et al. 2003). Additionally, age-mates and litter-mates that formed long-term alliances 
rose in rank together using coalitionary aggression. These observations would fit to the pattern observed with rank-changing coalitions between bonded individuals, although as the authors note future studies on male wild dogs would be required to corroborate these findings. The social systems of bottlenose dolphins (Tursiops aduncus) and hyenas show similar complexity to multi-male multi-female primate groups (Smith et al. 2010) and thus, may promote the frequent within group coalition formation observed in both species. In hyenas the majority of these coalitions are between female group members (Engh et al. 2005; Smith et al. 2010), females are the dominant sex which may inhibit male coalition formation and males queue for higher rank position. Bottlenose dolphin males form long-term alliances and these bonded males either gain access to or defend fertile females from other group males (Connor et al. 1992; Connor 2001; Frère et al. 2010), comparable to primate levelling coalitions (Connor et al. 1992). Male dolphin associations can also show great stability (Connor et al. 1992; 1999) and males with greater bond stability also had greater consortship rates than males with lesser bond stability. Similar to nonhuman primates where males with stronger social bonds gain increased reproductive benefits (Schülke et al. 2010; Gilby et al. 2012). These studies in non-primate mammalian species highlight the range of taxa with which cooperation between group members can manifest itself through coalitionary aggression and the potential reproductive benefits males can gain through cooperating.

\subsection{Cooperation in primate societies}

Cooperation is proposed to have developed via kin selection where the cooperating individuals are related (Hamilton 1963; 1964a; 1964b). For the majority of mammalian species, males are the dispersing sex (Johnson 1986) and thus, may not reside with kin or if kin are present it may prove difficult to recognise relatives. Kin selection theory (Hamilton 1963; 1964a; 1964b) may be limited in its explanation of the evolution of cooperation in male primate societies, as males in many groups are likely to be unaware of the presence of relatives. This is highlighted by research in Assamese macaques where males disperse from there natal groups but relatives were still present (Schülke et al. 2010). However, males formed strong bonds between non-kin and these strongly bonded individuals were more frequent coalition partners. In chimpanzees where males are the philopatric sex, males formed strong bonds with other group members who were not necessarily kin (Langergraber et al. 2007; Mitani 2009) and males who regularly affiliated also formed coalitions frequently (Watts 2002), suggesting that relatedness may not be a prerequisite for social bond development or cooperation between male primates. Barbary macaque males are the dispersing sex and also form strong bonds (Chapter 5 ) and these strongly bonded males are more likely to be recruited for coalition formation. Although relatedness 
between males was beyond the scope of this study it may well be that kin-relations and recognition are similar to the closely related Assamese macaques, although further investigation is required.

Cooperation between non-kin may be more likely to evolve if there are mutual benefits for both partners (Clutton-Brock 2009a). If both individuals gain immediate benefits of cooperating then defection will not provide benefits to cheaters and cooperation may be more likely to evolve (Clutton-Brock 2009a). As both individuals gain from acting cooperatively then group members should exchange short-term commodities to gain benefits (Noë et al. 1991; Noë and Hammerstein 1994; 1995) and select partners which provide the greatest payoffs. Thus, the dynamic nature of multi-male, multi-female societies, where many different group mates can provide competing commodities, may fit with partner choice models (Bshary and Noë 2003; Noë and Völkl 2013). Individuals within groups can base their partner choice decisions on previous experiences with other group members based on attitudinal partner choice (Noë and Völkl 2013). The development and maintenance of social bonds between partners can lead to positive associations bewteen partners and trust can develop between dyads with strong social bonds. Dyads with strong bonds can more readily provide support in agonistic situations creating a positive feedback loop to further enhance social bond strength. The pool of alternative partners within relatively stable groups may also discourage defection by one partner of a cooperative dyad because if defection occurred then their partner can switch to a new preferred ally (Noë and Völkl 2013).

Overall, cooperation in the form of within group male-male coalition formation may occur in primate groups via two main pathways contingent on the within group contest potential (illustrated in Figure 6.1). Where contest competition is high between males, coalition partners can both gain mutual benefits from breaking up consortships and partner choice will be opportunistic and situation dependent (Bercovitch 1988; Noë and Sluijter 1990; Noë 1992; 1994). Alternatively, mid-low contest potential can provide an environment whereby males still aspire to reach high dominance status to gain greater reproductive success. Cooperation requires a reliable partner which may be mediated by social bonds (Ostner and Schülke submitted). Emphasised by this study (Chapter 5 ) where males are more likely to recruit strongly bonded partners in agonistic aggression from the bystanders available. Affiliation between nonkin can foster the development of social bonds, leading to regular partner choice in high risk cooperative acts via attitudinal partner choice (Noë and Völkl 2013; Ostner and Schülke submitted). 


\subsection{Outlook}

By adopting a bottom-up approach this thesis unites several previously separate pieces of research into one comprehensive picture. In this study, I investigated the complete chain of evidence starting with understanding what males can infer about female reproductive state, before examining male contest competition and mating skew, looking at how male affiliation leads to social bonding between males and ultimately how this all ties into male cooperation through coalition formation. Male Barbary macaques cannot distinguish female conception and post-conception swelling periods, extending female receptivity. Male mating behaviour is skewed up the hierarchy but female receptive synchrony, female mating behaviour and male coalitionary activity all influence male mating success. Barbary macaque males formed long-term social bonds enduring highly competitive mating seasons. Males who shared strong social bonds were more likely to recruit each other as a coalition partner suggesting that partner choice is based on more than rank position alone. Coalitionary activity can provide mutual benefits for both partners suggesting that cooperation may be driven by previous affiliative interactions with group members brought about by attitudinal partner choice.

Ultimately, in order to fully understand the evolutionary mechanisms behind cooperation between non-kin it is vital to gain long-term data and attain true measures of male reproductive success via paternity analysis. This study highlights the effectiveness of using a bottom-up approach to examine male reproductive tactics, firstly, investigating male knowledge of female reproductive state via mating success to the use of cooperative strategies to increase reproductive success. By examining factors which can cause deviations from the expected mating/reproductive skew predicted by the PoA model, future research may be able to shed new light on male and female alternative reproductive strategies. Understanding the physiological costs of strong/weak social bonding and within group contest is a vital step in exploring the chain of events leading to costs and benefits of sociality between males. A comparative analysis across mammalian species may help to disentangle the evolutionary path of coalitionary aggression, for example, if large brain size and greater cognitive abilities are the main driving force behind coalition formation (Dunbar and Shultz 2007). This may help explaining the high proportion of primate species expressing coalition formation compared to other mammalian species. Finally, recent evidence suggests males who form strong social bonds with the alpha male and support the alpha male in coalitionary aggression can gain additional benefits within the group. For example, mating concessions (Duffy et al. 2007; Snyder-Mackler et al. 2012) or feeding tolerance (Berman et al. 2007). By examining these possible additional benefits across a broad range of species, including those where such benefits may be absent 
may shed further light on the mechanistic dynamic of social bond formation, maintenance and cooperation.

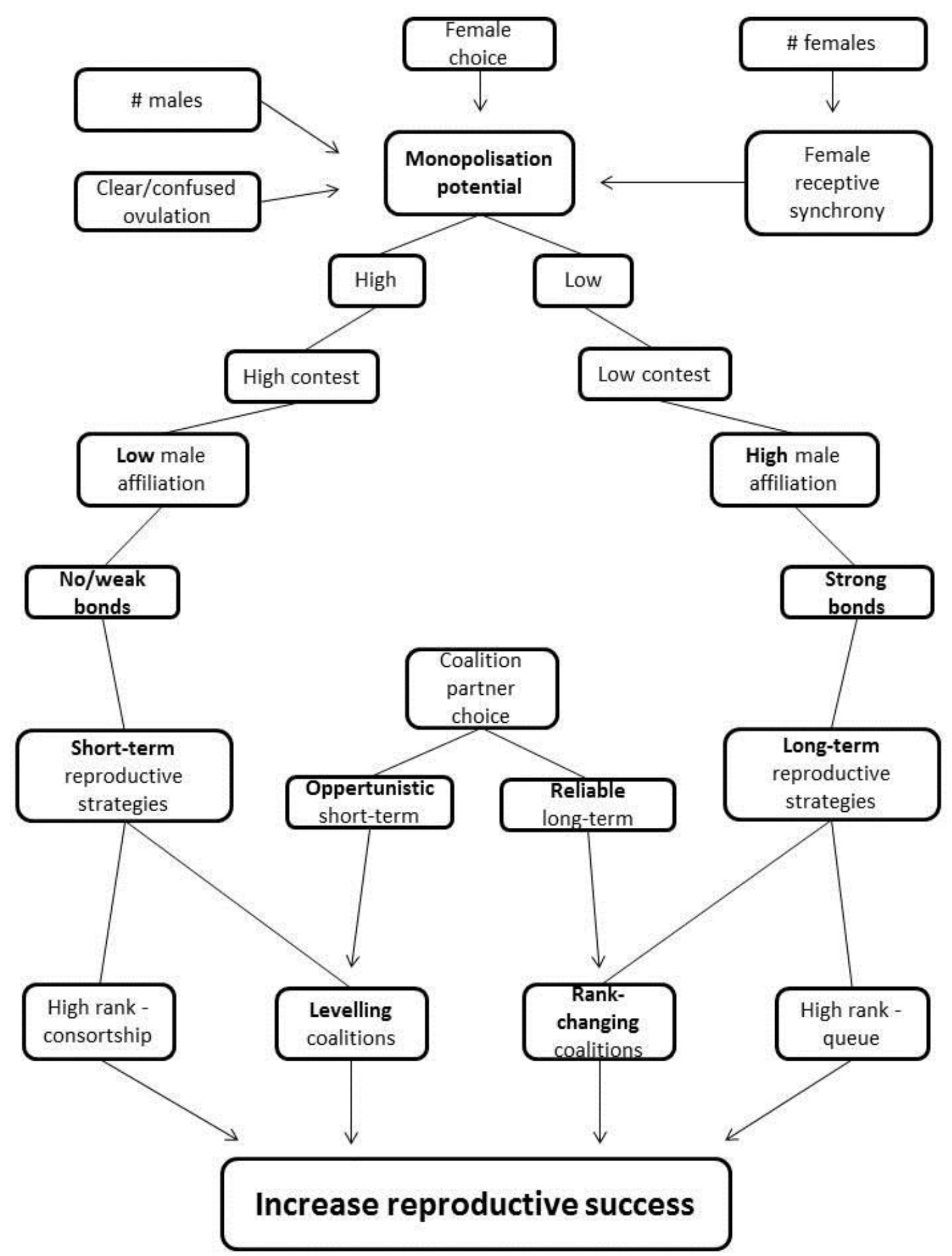

Figure 6.1: Chain of events by which males can gain greater reproductive success depending on the level of monopolisation potential and factors which influence monopolisation potential are shown. 


\section{References}

Alberts S (2012) Magnitude and sources of variation in male reproductive performance. In: Mitani JC, Call J, Kappeler PM, Palombit RA, Silk JB (eds) The evolution of primate societies. The University of Chicago Press, Chicago, pp 412-431

Alberts S, Altmann J, Wilson M (1996) Mate guarding constrains foraging activity of male baboons. Animal Behaviour 51:1269-1277

Alberts S, Buchan J, Altmann J (2006) Sexual selection in wild baboons: from mating opportunities to paternity success. Animal Behaviour 72:1177-1196

Alberts S, Watts H, Altmann J (2003) Queuing and queue-jumping: long-term patterns of reproductive skew in male savannah baboons, Papio cynocephalus. Animal Behaviour 65:821-840

Altmann J (1974) Observational study of behavior: sampling methods. Behaviour 49:227-267

Altmann SA (1962) A field study of the sociobiology of the rhesus monkey, Macaca mulatta. Annals of the New York Academy of Sciences 102:338-435

Armitage KB, Schwartz OA (2000) Social enhancement of fitness in yellow-bellied marmots. Proceedings of the National Academy of Sciences 97:12149-12152

Aujard F, Heistermann M, Thierry B, Hodges JK (1998) Functional significance of behavioral, morphological and endocrine correlates across the ovarian cycle in semifree ranging female Tonkean macaques. American Journal of Primatology 46:285-309

Baayen HR (2008) Analyzing linguistic data. A practical introduction to statistics using R. Cambridge University Press, Cambridge

Barclay P (2013) Strategies for cooperation in biological markets, especially for humans. Evolution and Human Behavior 34:164-175

Barelli C, Heistermann M, Boesch C, Reichard UH (2008) Mating patterns and sexual swellings in pair-living and multimale groups of wild white-handed gibbons, Hylobates lar. Animal Behaviour 75:991-1001

Barrett L, Henzi P (2001) The utility of grooming in baboon troops. In: Noe R, Van Hooff JARAM, Hammestein P (eds) Economics in Nature - Social Dilemmas, Mate Choice and Biological Markers. Cambridge Universtiy Press, Cambridge, pp 119 - 145

Barrett L, Henzi P, Rendall D (2007) Social brains, simple minds: does social complexity really require cognitive complexity? Philosophical Transactions of the Royal Society B: Biological Sciences 362:561-575

Barrett L, Henzi S (2006) Monkeys, markets and minds: biological markets and primate sociality. In: Kappeler $\mathrm{P}$, van Schaik $\mathrm{C}$ (eds) Cooperation in primates and humans. Springer, Heidelberg, pp 209-232

Barrett L, Henzi S, Weingrill T, Lycett J, Hill R (1999) Market forces predict grooming reciprocity in female baboons. Proceedings of the Royal Society London B 266:665-670

Bates D, Maechler M (2010) Ime4: Linear mixed-effects models using S4 classes. In: R package version 0.999375-37

Bates D, Maechler M, Bolker B (2012) Ime4 - linear mixed effects models using S4 classes. In: R package version 0.999999-0. http://CRAN.R-project.org/package=Ime4

Beehner J, Bergman T, Cheney D, Seyfarth R, Whitten P (2005) The effect of new alpha males on female stress in free-ranging baboons. Animal Behaviour 69:1211-1221

Behboodi E, Katz DF, Samuels SJ, Tell L, Hendrickx AG, Lasley BL (1991) The use of a urinary estrone conjugates assay for detection of optimal mating time in the cynomolgus macaque (Macaca fascicularis). Journal of Medical Primatology 20:229-234

Berard J, Nurnberg P, Epplen J, Schmidtke J (1994) Alternative reproductive tactics and reproductive success in male rhesus macaques. Behaviour 129:177 - 201 
Bercovitch FB (1988) Coalitions, cooperation and reproductive tactics among adult male baboons. Animal Behaviour 36:1198-1209

Berghänel A, Ostner J, Schröder U, Schülke O (2011a) Social bonds predict future cooperation in male Barbary macaques, Macaca sylvanus. Animal Behaviour 81:1109-1116

Berghänel A, Ostner J, Schülke O (2011b) Coalitions destabilize dyadic dominance relationships in male Barbary macaques (Macaca sylvanus). Behaviour 148:1257-1257

Berghänel A, Schülke O, Ostner J (2010) Coalition formation among Barbary macaque males: the influence of scramble competition. Animal Behaviour 80:675-682

Berman C, lonica C, Li J (2007) Supportive and tolerant relationships among male Tibetan macaques at Huangshan, China. Behaviour 144:631-661

Bielert C, Anderson CM (1985) Baboon sexual swellings and male response: a possible operational mammalian supernormal stimulus and response mechanism. International Journal of Primatology 6:377-393

Bielert C, Czaja JA, Eisele S, Scheffler G, Robinson JA, Goy RW (1976) Mating in the rhesus monkey (Macaca mulatta) after conception and its relationship to oestradiol and progesterone levels throughout pregnancy. Journal of Reproduction and Fertility 46:179187

Bissonnette A (2009) Testing a model on coalitions in Barbary macaque males (Macaca sylvanus). PhD Thesis. Univeristy of Zürich, Zürich

Bissonnette A, Bischofberger N, van Schaik C (2011) Mating skew in Barbary macaque males: the role of female mating synchrony, female behavior, and male-male coalitions. Behavioral Ecology and Sociobiology 65:167-182

Bissonnette A, de Vries H, van Schaik CP (2009a) Coalitions in male Barbary macaques, Macaca sylvanus: strength, success and rules of thumb. Animal Behaviour 78:329-335

Bissonnette A, Lange E, Van Schaik CP (2009b) A cardinal measure of competitive ability in Barbary macaque males (Macaca sylvanus). Ethology 115:671-681

Blundell G, Ben-David M, Groves P, Bowyer R, Geffen E (2004) Kinship and sociality in coastal river otters: are they related? Behavioral Ecology 15:705- 714

Boehm C (1993) Cooperation and prosocial behaviour. American Anthropologist 95:455-456

Boehm C (1999) Hierarchy in the forest - the evolution of egalitarian behavior. Harvard University Press, Cambridge, Massachussetts

Boesch C (1994a) Chimpanzees-red colobus monkeys: a predator-prey system. Animal Behaviour 47:1135-1148

Boesch C (1994b) Cooperative hunting in wild chimpanzees. Animal Behaviour 48:653-667

Boesch C, Boesch-Achermann H (2000) The chimpanzees of the Tai Forest: behavioural ecology and evolution. Oxford University Press, New York

Boesch C, Kohou G, Nene H, Vigilant L (2006) Male competition and paternity in wild chimpanzees of the Taï Forest. American Journal of Physical Anthropology 130:103-115

Boinski S (1987) Mating patterns in squirrel monkeys (Saimiri oerstedi). Behavioral Ecology and Sociobiology 21:13-21

Borries C (2000) Male dispersal and mating season influxes in Hanuman langurs living in multimale groups. In: Kappeler PM (ed) Primate males: causes and consequences of variation in group composition. Cambridge University Press, Cambridge, pp 146-158

Bowerman B, O'Connell R (1990) Linear statistical models: An applied approach, 2nd edn. Duxbury, Belmont, CA

Bowles R (2007) Genetic diffentiation among hunter-gatherer groups: implications for ancestral human social structure. In. Santa Fe Institute, pp 25

Brauch K, Hodges K, Engelhardt A, Fuhrmann K, Shaw E, Heistermann M (2008) Sex-specific reproductive behaviours and paternity in free-ranging Barbary macaques (Macaca sylvanus). Behavioral Ecology and Sociobiology 62:1453-1466

Brauch K, Pfefferle D, Hodges K, Möhle U, Fischer J, Heistermann M (2007) Female sexual behavior and sexual swelling size as potential cues for males to discern the female fertile 
phase in free-ranging Barbary macaques (Macaca sylvanus) of Gibraltar. Hormones and Behavior 52:375- 383

Braun A, Bugnyar T (2012) Social bonds and rank acquisition in raven nonbreeder aggregations. Animal Behaviour 84:1507-1515

Broom M, Koenig A, Borries C (2009) Variation in dominance hierarchies among group-living animals: modeling stability and the likelihood of coalitions. Behavioral Ecology 20:844855

Brosnan S, Waal FM (2002) A proximate perspective on reciprocal altruism. Human Nature 13:129-152

Bshary R, Noë R (2003) Biological markets: The ubiquitous influence of partner choice on the dynamics of cleaner fish - client reef fish interactions. In: Hammerstein P (ed) Genetic and Cultural Evolution of Cooperation. MIT Press, Cambridge, MA, pp 167-184

Bulger J (1993) Dominance rank and access to estrous females in male savanna baboons. Behaviour 127:67-103

Cameron E, Setsaas T, Linklater W (2009) Social bonds between unrelated females increase reproductive success in feral horses. Proceedings of the National Academy of Sciences 106:13850

Carnegie SD, Fedigan LM, Ziegler TE (2005) Behavioral indicators of ovarian phase in white-faced capuchins (Cebus capucinus). American Journal of Primatology 67:51-68

Caro TM, Collins DA (1987) Male cheetah social organization and territoriality. Ethology 74:52-64

Cerda-Molina A, Hernandez-Lopez L, Chavira R, Cardenas M, Paez-Ponce D, Cervantes-De la Luz $\mathrm{H}$, Mondragon-Ceballos R (2006) Endocrine changes in male stumptailed macaques (Macaca arctoides) as a resonse to odor stimulation with vaginal secretions. Hormones and Behavior 49:81-87

Chapais B (1995) Alliances as means of competition in primates: evolutionary, developmental and cognitive aspects. Yearbook of Physical Anthropology 38:115-136

Cheney DL (2011) Extent and limits of cooperation in animals. Proceedings of the National Academy of Sciences 108:10902-10909

Clarke PMR, Henzi SP, Barrett L (2009) Sexual conflict in chacma baboons, Papio hamadryas ursinus: absent males select for proactive females. Animal Behaviour 77:1217-1225

Clutton-Brock T (2009a) Cooperation between non-kin in animal societies. Nature 462:51-57

Clutton-Brock $T$ (2009b) Structure and function in mammalian societies. Philosophical Transactions of the Royal Society B: Biological Sciences 364:3229-3242

Clutton-Brock T, Parker G (1995) Punishment in animal societies. Nature 373:209-216

Clutton-Brock TH, Lukas D (2011) The evolution of social philopatry and dispersal in female mammals. Molecular Ecology 21:472-492

Clutton-Brock TH, Parker GA (1992) Potential reproductive rates and the operation of sexual selection. The Quarterly Review of Biology 67:437-456

Coltman DW, Bowen WD, Boness DJ, Iverson SJ (1997) Balancing foraging and reproduction in the male harbour seal, an aquatically mating pinniped. Animal Behaviour 54:663-678

Connor R, Smolker R, Richards A (1992) Dolphin alliances and coalitions. In: Harcourt A, De Waal $F$ (eds) Coalitions and alliances in humans and other animals. Oxford University Press, Oxford, pp 415-444

Connor R, Whitehead H (2005) Alliances II. Rates of encounter during resource utilization: a general model of intrasexual alliance formation in fission-fusion societies. Animal Behaviour 69:127-132

Connor RC (2001) Bottlenose dolphins: Social relationships in a big-brained aquatic mammal. In: Dugatkin LA (ed) Model Systems in Behavioral Ecology: Integrating Conceptual, Theoretical, and Empirical Approaches. Princeton University Press, Princeton, pp 408432

Connor RC, Heithaus MR, Barre LM (1999) Superalliance of bottlenose dolphins. Nature 397:571572 
Connor RC, Heithaus MR, Barre LM (2001) Complex social structure, alliance stability and mating access in a bottlenose dolphin "super-alliance". Proceedings of the Royal Society of London. Series B: Biological Sciences 268:263-267

Cords M (1997) Friendships, alliances, reciprocity and repair. In: Whiten A, Byrne R (eds) Machiavellian intelligence II. Cambridge University Press, Cambridge, pp 24-49

Cords M (2000) The number of males in guenon groups. In: Kappeler PM (ed) Primate males: causes and consequences of variation in group composition. Cambridge University Press, Cambridge, pp 84-96

Cowlishaw G, Dunbar RIM (1991) Dominance rank and mating success in male primates. Animal Behaviour 41:1045-1056

Crockford C, Wittig RM, Langergraber K, Ziegler TE, Zuberbühler K, Deschner T (2013) Urinary oxytocin and social bonding in related and unrelated wild chimpanzees. Proceedings of the Royal Society B: Biological Sciences 280

Crockford C, Wittig RM, Whitten P, Seyfarth RM, Cheney DL (2008) Social stressors and coping mechanisms in wild female baboons (Papio hamadryas ursinus). Hormones and Behavior 53:254-265

de Villiers MS, Richardson PRK, van Jaarsveld AS (2003) Patterns of coalition formation and spatial association in a social carnivore, the African wild dog (Lycaon pictus). Journal of Zoology 260:377-389

de Vries H, Netto WJ, Hanegraaf PLH (1993) MATMAN: a program for the analysis of sociometric matrices and behavioural transition matrices. Behaviour 125:157-175

de Vries $\mathrm{H}$, Stevens J, Vervaecke $\mathrm{H}$ (2006) Measuring and testing the steepness of dominance hierarchies. Animal Behaviour 71:585-592

de Waal F, Harcourt A (1992) Coalitions and alliances: a history of ethological research. In: Harcourt A, de Waal $F$ (eds) Coalitions and alliances in humans and other animals. Oxford University Press, Oxford, pp 1-19

de Waal FBM (2000) Attitudinal reciprocity in food sharing among brown capuchin monkeys. Animal Behaviour 60:253-261

Deag J (1980) Interactions between males and unweaned Barbary macaques: Testing the agonistic buffering hypothesis. Behaviour 75:54-81

Deag J, Crook J (1971) Social behaviour and "agonistic buffering" in the wild Barbary macaque, Macaca sylvana L. Folia Primatologica 15:183-200

Deag JM (1974) A study of the social behaviour and ecology of the wild Barbary macaque Macaca sylvanus. PhD thesis, University of Bristol, Bristol, UK

Deschner T, Heistermann M, Hodges J, Boesch C (2003) Timing and probability of ovulation in relation to sex skin swelling in wild West African chimpanzees, Pan troglodytes verus. Animal Behaviour 66:551-560

Deschner T, Heistermann M, Hodges JK, Boesch C (2004) Female sexual swelling size, timing of ovulation, and male behavior in wild West African chimpanzees. Hormones and Behavior 46:204-215

DeScioli P, Kurzban R (2009) The alliance hypothesis for human friendship. PLoS ONE 4:e5802

Dewsbury D (1982) Dominance rank, copulatory behavior, and differential reproduction. The Quarterly Review of Biology 57:135-159

Dixson AF (1998) Primate Sexuality: Comparative Studies of the Prosimians, Monkeys, Apes, and Human Beings, 1st edn. Oxford University Press, Oxford

Dixson AF (2012) Primate Sexuality: Comparative Studies of the Prosimians, Monkeys, Apes, and Humans, 2nd edn. Oxford University Press, Oxford

Drea CM (2005) Bateman revisited: the reproductive tactics of female primates. Integrative and Comparative Biology 45:915-923

Drews C (1993) The concept and definition of dominance in animal behaviour. Behaviour $125: 283-311$ 
Dubuc C, Muniz L, Heistermann M, Engelhardt A, Widdig A (2011) Testing the priority-of-access model in a seasonally breeding primate species. Behavioral Ecology and Sociobiology 65:1615-1627

Duffy KG, Wrangham RW, Silk JB (2007) Male chimpanzees exchange political support for mating opportunities. Current Biology 17:R586-R587

Dufour V, Pelé M, Neumann M, Thierry B, Call J (2009) Calculated reciprocity after all: computation behind token transfers in orang-utans. Biology Letters 5:172-175

Dugatkin LA (1997) The evolution of cooperation. Bioscience 47:355-362

Dugatkin LA (1998) Breaking up fights between others: a model of intervention behaviour. Proceedings of the Royal Society of London. Series B: Biological Sciences 265:433-437

Dunbar R (1991) Functional significance of social grooming in primates. Folia Primatologica $57: 121-131$

Dunbar R (1998) The social brain hypothesis. Evolutionary Anthropology 6:178-190

Dunbar R, Shultz S (2007) Understanding primate brain evolution. Philosophical Transactions of the Royal Society B: Biological Sciences 362:649-658

Dunbar RIM (1992) Time: a hidden constraint on the behavioural ecology of baboons. Behavioral Ecology and Sociobiology 31:35-49

Dunbar RIM (1995) Neocortex size and group size in primates: a test of the hypothesis. Journal of Human Evolution 28:287-296

Dunbar RIM, Korstjens AH, Lehmann J, British Academy Centenary Research P (2009) Time as an ecological constraint. Biological Reviews 84:413-429

Ellis $L$ (1995) Dominance and reproductive success among nonhuman animals: a cross-species comparison. Ethology and Sociobiology 16:257-333

Emery M, Whitten P (2003) Size of sexual swellings reflects ovarian function in chimpanzees (Pan troglodytes). Behavioral Ecology and Sociobiology 54:340-351

Emlen DJ (2008) The evolution of animal weapons. Annual Review of Ecology, Evolution, and Systematics 39:387-413

Engelhardt A, Hodges J, Heistermann M (2007) Post-conception mating in wild long-tailed macaques (Macaca fascicularis): Characterization, endocrine correlates and functional significance. Hormones and Behavior 51:3-10

Engelhardt A, Hodges JK, Niemitz C, Heistermann M (2005) Female sexual behavior, but not sex skin swelling reliably indicates the timing of the fertile phase in wild long-tailed macaques (Macaca fascicularis). Hormones and Behavior 47:195-204

Engelhardt A, Pfeifer J-B, Heistermann M, Niemitz C, van Hooff J, Hodges J (2004) Assessment of female reproductive status by male longtailed macaques, Macaca fascicularis, under natural conditions. Animal Behaviour 67:915-924

Engh A, Beehner J, Bergman T, Whitten P, Hoffmeier R, Seyfarth RM, Cheney DL (2006) Female hierarchy instability, male immigration and infanticide increase glucocorticoid levels in female chacma baboons. Animal Behaviour 71:1227-1237

Engh A, Funk S, van Horn R, Scribner K, Bruford M, Libants S, Szykman M, Smale L, Holekamp K (2002) Reproductive skew among males in a female-dominated mammalian society. Behavioral Ecology 13:193- 200

Engh AL, Siebert ER, Greenberg DA, Holekamp KE (2005) Patterns of alliance formation and postconflict aggression indicate spotted hyaenas recognize third-party relationships. Animal Behaviour 69:209-217

Fedigan L, Jack K (2004) The demographic and reproductive context of male replacements in cebus capucinus. Behaviour 141:755- 775

Feh C (1999) Alliances and reproductive success in Camargue stallions. Animal Behaviour 57:705713

Field A (2005) Discovering statistics using SPSS. Sage Publications, London

Fischer J, Hammerschmidt K (2002) An Overview of the Barbary Macaque, Macaca sylvanus, Vocal Repertoire. Folia Primatologica 73:32-45 
Fooden J (2007) Systematic review of the Barbary macaque, Macaca sylvanus (Linnaeus, 1758). In. Field Museum of Natural History, Chicago, III

Fox J, Weisberg HS (2010) An R companion to applied regression. Sage Publications, Thousand Oaks, CA

Fraser ON, Bugnyar T (2010) The quality of social relationships in ravens. Animal Behaviour 79:927-933

Frère CH, Krützen M, Mann J, Connor RC, Bejder L, Sherwin WB (2010) Social and genetic interactions drive fitness variation in a free-living dolphin population. Proceedings of the National Academy of Science 107:19949-19954

Fürtbauer I, Heistermann M, Schülke O, Ostner J (2011a) Concealed fertility and extended female sexuality in a non-human primate (Macaca assamensis). PLoS ONE 6:e23105

Fürtbauer I, Mundry R, Heistermann M, Schülke O, Ostner J (2011b) You mate, I mate: macaque females synchronize sex not cycles. PLoS ONE 6:e26144

Fürtbauer I, Schülke O, Heistermann M, Ostner J (2010) Reproductive and life history parameters of wild female Assamese macaques (Macaca assamensis). International Journal of Primatology 31:501-517

Gilby I, Brent LN, Wroblewski E, Rudicell R, Hahn B, Goodall J, Pusey A (2012) Fitness benefits of coalitionary aggression in male chimpanzees. Behavioral Ecology and Sociobiology:1-9

Gilby IC (2012) Cooperation among Non-kin. In: Mitani JC, Call J, Kappeler PM, Palombit RA, Silk JB (eds) The evolution of primate societies. The University of Chicago Press, Chicago, pp 514-530

Gogarten J, Koenig A (2012) Reproductive seasonality is a poor predictor of receptive synchrony and male reproductive skew among nonhuman primates. Behavioral Ecology and Sociobiology:1-12

Goldberg TL, Wrangham RW (1997) Genetic correlates of social behaviour in wild chimpanzees: evidence from mitochondrial DNA. Animal Behaviour 54:559-570

Gordon T, Gust D, Busse C, Wilson M (1991) Hormones and sexual behavior associated with postconception perineal swelling in the sooty mangabey (Cercocebus torquatus atys). International Journal of Primatology 12:585-597

Gouzoules H, Gouzoules S (1995) Recruitment screams of pigtail monkeys (Macaca nemestrina): ontogenetic perspectives. Behaviour:431-450

Grinnell J, Packer C, Pusey AE (1995) Cooperation in male lions: kinship, reciprocity or mutualism? Animal Behaviour 49:95-105

Gust D (1994) Alpha-male sooty mangabeys differentiate between females' fertile and their postconception maximal swellings. International Journal of Primatology 15:289-301

Hamilton W (1964a) The genetical evolution of social behaviour. I. Journal of Theoretical Biology 7:1-16

Hamilton W (1964b) The genetical evolution of social behaviour. II. Journal of Theoretical Biology 7:17-52

Hamilton WD (1963) The evolution of altruistic behavior. The American Naturalist 97:354-356

Harcourt A (1992) Coalitions and alliances: are primates more complex than non-primates? In: Harcourt A, De Waal F (eds) Coalitions and alliances in humans and other animals. Oxford University Press, Oxford pp 445-472

Harcourt A, de Waal F (1992) Coalitions and alliances in humans and other animals. Oxford University Press, Oxford

Hayakawa S (2008) Male-female mating tactics and paternity of wild Japanese macaques (Macaca fuscata yakui). American Journal of Primatology 70:986-989

Heistermann M, Brauch K, Möhle U, Pfefferle D, Dittami J, Hodges K (2008) Female ovarian cycle phase affects the timing of male sexual activity in free-ranging Barbary macaques (Macaca sylvanus) of Gibraltar. American Journal of Primatology 70:44-53

Heistermann M, Finke M, Hodges JK (1995) Assessment of female reproductive status in captivehoused Hanuman langurs (Presbytis entellus) by measurement of urinary and fecal steroid excretion patterns. American Journal of Primatology 37:275-284 
Heistermann M, Möhle U, Vervaecke H, van Elsacker L, Hodges JK (1996) Application of urinary and fecal steroid measurements for monitoring ovarian function and pregnancy in the Bonobo (Pan paniscus) and evaluation of perineal swelling patterns in relation to endocrine events. Biology of Reproduction 55:844-853

Heistermann M, Ziegler T, van Schaik CP, Launhardt K, Winkler P, Hodges JK (2001) Loss of oestrus, concealed ovulation and paternity confusion in free-ranging Hanuman langurs. Proceedings of the Royal Society London B 268:2445-2451

Hemelrijk C (1990) Models of, and tests for, reciprocity, unidirectionality and other social interaction patterns at a group level. Animal Behaviour 39:1013-1029

Hemelrijk C, Ek A (1991) Reciprocity and interchange of grooming and 'support' in captive chimpanzees. Animal Behaviour 41:923-935

Hemelrijk CK, Puga-Gonzalez I (2012) An individual-oriented model on the emergence of support in fights, its reciprocation and exchange. PLoS ONE 7:e37271

Henkel S, Heistermann M, Fischer J (2010) Infants as costly social tools in male Barbary macaque networks. Animal Behaviour 79:1199-1204

Henzi P, Barrett L (2003) Evolutionary ecology, sexual conflict, and behavioral differentiation among baboon populations. Evolutionary Anthropology 12:217-230

Henzi SP, Barrett $L$ (1999) The value of grooming to female primates. Primates 40:47-59

Henzi SP, Clarke PMR, van Schaik CP, Pradhan GR, Barrett L (2010) Infanticide and reproductive restraint in a polygynous social mammal. Proceedings of the National Academy of Science 107:2130-2135

Henzi SP, Lusseau D, Weingrill T, Schaik CP, Barrett $L$ (2009) Cyclicity in the structure of female baboon social networks. Behavioral Ecology and Sociobiology 63:1015-1021

Hesler N, Fischer J (2007) Gestural communication in Barbary macaques (Macaca sylvanus): An overview. In: Tomasello M, Call J (eds) The gestural communication in apes and monkeys. Mahwah: Lawrence Erlbaum Associates, pp 159-195

Higham JP, Heistermann M, Ross C, Semple S, MacLarnon A (2008a) The timing of ovulation with respect to sexual swelling detumescence in wild olive baboons. Primates 49:295-299

Higham JP, Heistermann M, Saggau C, Agil M, Perwitasari-Farajallah D, Engelhardt A (2012) Sexual signalling in female crested macaques and the evolution of primate fertility signals. BMC Evolutionary Biology 12:89

Higham JP, MacLarnon AM, Ross C, Heistermann M, Semple S (2008b) Baboon sexual swellings: Information content of size and color. Hormones and Behavior 53:452-462

Higham JP, Maestripieri D (2010) Revolutionary coalitions in male rhesus macaques. Behaviour 147:1889-1908

Higham JP, Semple S, MacLarnon A, Heistermann M, Ross C (2009) Female reproductive signaling, and male mating behavior, in the olive baboon. Hormones and Behavior 55:6067

Hinde R (1976) Interactions, relationships and social structure. Man 11:1-17

Hinde RA (1983) Primate social relationships: An integrated approach. Blackwell Scientific Publications, Oxford

Hirotani A (1994) Dominance rank, copulatory behaviour and estimated reproductive success in male reindeer. Animal Behaviour 48:929- 936

Hodges JK, Heistermann M, Beard A, van Aerde RJ (1997) Concentrations of progesterone and the $5 \alpha$-reduced progestins, $5 \alpha$-pregnane-3,20-dione and $5 \alpha$-pregnane-3 $\alpha$-ol-20-one in luteal tissue and circulating blood and their relationship to luteal function in the African elephant, Loxodonta africana. Biology of Reproduction 56:640-646

Holt-Lunstad J, Smith TB, Layton JB (2010) Social relationships and mortality risk: a meta-analytic review. PLoS Med 7:e1000316

Hrdy SB, Whitten PL (1987) Patterning of sexual activity. In: Smuts BB, Cheney DL, Seyfarth RM, Wrangham RW, Struhsaker TT (eds) Primate Societies. The University of Chicago Press, Chicago, pp 370-384 
Huffman M, A. (1987) Consort intrusion and female mate choice in Japanese macaques (Macaca fuscata). Ethology 75:221-234

Isbell LA (1991) Contest and scramble competition: patterns of female aggression and ranging behavior in primates. Behavioral Ecology:143-155

Janson C (1984) Female choice and mating system of the brown capuchin monkey Cebus apella (primates: Cebidae). Zeitschrift für Tierpsychologie 65:177-200

Jennings DJ, Carlin CM, Gammell MP (2009) A winner effect supports third-party intervention behaviour during fallow deer, Dama dama, fights. Animal Behaviour 77:343-348

Jennings DJ, Carlin CM, Hayden TJ, Gammell MP (2011) Third-party intervention behaviour during fallow deer fights: the role of dominance, age, fighting and body size. Animal Behaviour 81:1217-1222

Johnson CN (1986) Sex-biased philopatry and dispersal in mammals. Oecologia 69:626-627

Johnstone RA, Dugatkin LA (2000) Coalition formation in animals and the nature of winner and loser effects. Proceedings of the Royal Society B: Biological Sciences 267:17-21

Kappeler PM (2012) Mate choice. In: Mitani JC, Call J, Kappeler P, Palombit RA, Silk JB (eds) The evolution of primate societies. University of Chicago Press, Chicago, pp 367-386

Koenig A, Borries C, Suarez S, Kreetiyutanont K, Prabnasuk J (2004) Socio-ecology of Phayre's leaf monkeys (Trachypithecus phayrei) at Phu Khieo Wildlife Sanctuary. Journal of Wildlife in Thailand 12:150-163

Kokko H, Lindström J (1997) Measuring the mating skew. American Naturalist 149:794-799

Kuester J, Paul A (1984) Female reproductive characteristics in semifree-ranging Barbary macaques (Macaca sylvanus L. 1758). Folia Primatologica 43:69-83

Kuester J, Paul A (1992) Influence of male competition and female mate choice on male mating success in Barbary macaques (Macaca sy/vanus). Behaviour 120:192 - 217

Kuester J, Paul A (1999) Male migration in Barbary macaques (Macaca sylvanus) at Affenberg Salem. International Journal of Primatology 20:85-106

Kulik L, Muniz L, Mundry R, Widdig A (2012) Patterns of interventions and the effect of coalitions and sociality on male fitness. Molecular Ecology 21:699-714

Kutsukake N, Clutton-Brock TH (2010) Grooming and the value of social relationships in cooperatively breeding meerkats. Animal Behaviour 79:271-279

Kutsukake N, Hasegawa T (2005) Dominance turnover between an alpha and a beta male and dynamics of social relationships in Japanese macaques. International Journal of Primatology 26:775-800

Kutsukake N, Nunn C (2006) Comparative tests of reproductive skew in male primates: the roles of demographic factors and incomplete control. Behavioral Ecology and Sociobiology 60:695-706

Kvarnemo C, Ahnesjö I (1996) The dynamics of operational sex ratios and competition for mates. Trends in Ecology and Evolution 11:404-408

Langergraber K, E, Mitani J, C, Watts D, P, Vigilant L (2013) Male-female socio-spatial relationships and reproduction in wild chimpanzees. Behavioral Ecology and Sociobiology 67:861-873

Langergraber K, Mitani J, Vigilant L (2007) The limited impact of kinship on cooperation in wild chimpanzees. Proceedings of the National Academy of Sciences 104:7786-7790

Le Boeuf BJ (1974) Male-male competition and reproductive success in elephant seals. American Zoologist 14:163-176

Leiva D, de Vries H (2011) Testing steepness of dominance hierarchies. http://CRAN.Rproject.org/package=steepness. In: $\mathrm{R}$ package version 0.2

Lisk RD, Ciaccio LA, Catanzaro C (1983) Mating behaviour of the golden hamster under seminatural conditions. Animal Behaviour 31:659-666

Loretto MC, Fraser ON, Bugnyar T (2012) Otogeny of social relations and coalition formation in common ravens (Corvus corax). International Journal of Comparative Psychology 25:180194 
Lu A, Beehner JC, Czekala NM, Borries C (2012) Juggling priorities: female mating tactics in Phayre's leaf monkeys. American Journal of Primatology 74:471-481

Lukas D, Clutton-Brock T (2012) Cooperative breeding and monogamy in mammalian societies. Proceedings of the Royal Society B: Biological Sciences 279:2151-2156

Mainguy J, Côté SD (2008) Age- and state-dependent reproductive effort in male mountain goats, Oreamnos americanus. Behavioral Ecology and Sociobiology 62:935-943

Majolo B, Lehmann J, de Bortoli Vizioli A, Schino G (2012) Fitness-related benefits of dominance in primates. American Journal of Physical Anthropology 147:652-660

Majolo B, McFarland R, Young C, Qarro M (2013) The effect of climatic factors on the activity budgets of Barbary macaques (Macaca sylvanus). International Journal of Primatology 34:500-514

Manson JH (1995) Do female rhesus macaques choose novel males? American Journal of Primatology 37:285-296

Massen JJM, Sterck EHM, de Vos H (2010) Close social associations in animals and humans: functions and mechanisms of friendship. Behaviour 147:1379-1412

Mayers R (1990) Classical and modern regression with applications, 2nd edn. Duxbury, Boston, MA

McCarthy M, Becker J (2002) Neuroendocrinology of sexual behavior in the female. In: Becker J, Breedlove S, Crews D, McCarthy M (eds) Behavioral endocrinology, 2nd edn. The MIT Press, Cambridge, pp 117-152

Melis AP, Semmann D (2010) How is human cooperation different? Philosophical Transactions of the Royal Society B: Biological Sciences 365:2663-2674

Menard N, Vallet D (1996) Demography and ecology of Barbary macaques (Macaca sylvanus) living in two different habitats. In: Fa J, Lindburg D (eds) Evolution and Ecology of Macaque Societies. Cambridge University Press, Cambridge, pp 106-131

Mitani J (2006a) Demographic influences on the behavior of chimpanzees. Primates 47:6-13

Mitani J (2009) Male chimpanzees form enduring and equtable social bonds. Animal Behaviour 77:633-640

Mitani J, Merriwether DA, Zhang CB (2000) Male affiliation, cooperation and kinship in wild chimpanzees. Animal Behaviour 59:885-893

Mitani JC (2006b) Reciprocal exchange in chimpanzees and other primates. In: Kappeler P, van Schaik C (eds) Cooperation in primates and humans. Springer, Heidelberg, pp 107-120

Mitani JC, Watts DP, Pepper JW, Merriwether DA (2002) Demographic and social constraints on male chimpanzee behaviour. Animal Behaviour 64:727-737

Modolo L, Martin RD, van Schaik CP, van Noordwijk MA, Krutzen M (2008) When dispersal fails: unexpected genetic separation in Gibraltar macaques (Macaca sylvanus). Molecular Ecology 17:4027-38

Möhle U, Heistermann M, Dittami J, Reinberg V, Hodges J (2005) Patterns of anogenital swelling size and their endocrine correlates during ovulatory cycles and early pregnancy in freeranging barbary macaques (Macaca sylvanus) of Gibraltar. American Journal of Primatology 66:351-368

Morales JC, Melnick DJ (1998) Phylogenetic relationships of macaques (Cercopithecidae: Macaca), as revealed by high resolution restriction site mapping of mitrochondrial ribosome genes. Journal of Human Evolution 34:1-23

Muller M, Wrangham R (2004) Dominance, cortisol and stress in wild chimpanzees (Pan troglodytes schweinfurthii). Behavioral Ecology and Sociobiology 55:332-340

Muller MN, Emery Thompson M, Kahlenberg SM, Wrangham RW (2011) Sexual coercion by male chimpanzees shows that female choice may be more apparent than real. Behavioral Ecology and Sociobiology 65:921-933

Muller MN, Kahlenberg SM, Emery Thompson M, Wrangham RW (2007) Male coercion and the costs of promiscuous mating for female chimpanzees. Proceedings of the Royal Society B: Biological Sciences 274:1009-14 
Muller MN, Wrangham RW (2009) Sexual coercion in primates and humans: An evolutionary perspective on male aggression against females. Harvard University Press, Cambridge, MA

Newton-Fisher NE, Emery Thompson M, Reynolds V, Boesch C, Vigilant L (2010) Paternity and social rank in wild chimpanzees (Pan troglodytes) from the Budongo Forest, Uganda. American Journal of Physical Anthropology 142:417-428

Nieuwenhuijsen K, De Neef K, Slob A (1986) Sexual behaviour during ovarian cycles, pregnancy and lactation in group-living stumptail macaques (Macaca arctoides). Human Reproduction 1:159-169

Nishida T (1983) Alpha status and agonsitic alliance in wild chimpanzees. Primates 24:318- 336

Noë R (1990) A veto game played by baboons: a challenge to the use of the Prisoner's Dilemma as a paradigm for reciprocity and cooperation. Animal Behaviour 39:78-90

Noë R (1992) Alliance formation among male baboons: shopping for profitable partners. In: Harcourt A, De Waal $F$ (eds) Coalitions and alliances in humans and other animals. Oxford University Press, Oxford, pp 285-322

Noë R (1994) A model of coalition formation among male baboons with fighting ability as the crucial parameter. Animal Behaviour 47:211-213

Noë R, Hammerstein P (1994) Biological markets: supply and demand determine the effect of partner choice in cooperation, mutualism and mating. Behavioral Ecology and Sociobiology 35:1-11

Noë R, Hammerstein P (1995) Biological markets. Trends in Ecology and Evolution 10:336-339

Noë R, Sluijter AA (1990) Reproductive tactics of male savanna baboons. Behaviour 113:117-169

Noë R, Sluijter AA (1995) Which adult male savanna baboons form coalitions? International Journal of Primatology 16:77-105

Noë R, van Schaik CP, van Hooff JARAM (1991) The market effect: an explanation for pay-off asymetries among collaboration animals. Ethology 87:97-118

Noë R, Völkl B (2013) Cooperation and biological markets: the power of partner choice. In: Sterelny K, Joyce R, Calcott B, Fraser B (eds) Cooperation and its Evolution. MIT Press, Cambridge, pp 131-151

Nonacs P (2000) Measuring and using skew in the study of social behavior and evolution. American Naturalist 156:577-589

Nonacs P (2003) Measuring the reliability of skew indices: is there one best index? Animal Behaviour 65:615-627

Nowak M (2006) Five rules for the evolution of cooperation. Science 314:1560- 1563

Nowak M, A., Tarnina C, E., Wilson E, O. (2011) Nowak et al. reply. Nature 471:(7339): E9-E10: http://dx.doi.org/10.1038/nature09836

Nunn CL (1999a) The evolution of exaggerated swellings in primates and the graded-signal hypothesis. Animal Behaviour 58:229-246

Nunn CL (1999b) The number of males in primate groups: a comparative test of the socioecological model. Behavioral Ecology and Sociobiology 46:1-13

Nunn CL, van Schaik CP, Zinner D (2001) Do exaggerated sexual swellings function in female mating competition in primates? A comparative test of the reliable indicator hypothesis. Behavioral Ecology 12:646-654

O'Neill A, Fedigan LM, Ziegler TE (2004) Ovarian cycle phase and same-sex mating behavior in Japanese macaque females. American Journal of Primatology 63:25-31

Olson LE, Blumstein DT (2009) A trait-based approach to understand the evolution of complex coalitions in male mammals. Behavioral Ecology

Ostner J, Chalise M, Koenig A, Launhardt K, Nikolei J, Podzuweit D, Borries C (2006) What Hanuman langur males know about female reproductive status. American Journal of Primatology 68:701- 712

Ostner J, Heistermann M, Schülke O (2008a) Dominance, aggression and physiological stress in wild male Assamese macaques (Macaca assamensis). Hormones and Behavior 54:613619 
Ostner J, Heistermann M, Schülke O (2011) Male competition and its hormonal correlates in Assamese macaques (Macaca assamensis). Hormones and Behavior 59:105-113

Ostner J, Nunn CL, Schülke O (2008b) Female reproductive synchrony predicts skewed paternity across primates. Behavioral Ecology 19:1150-1158

Ostner J, Schülke O (submitted) The evolution of social bonds in primate males. Behaviour

Overduin-de Vries AM, Olesen CU, Vries H, Spruijt BM, Sterck EHM (2012) Sneak copulations in long-tailed macaques (Macaca fascicularis): no evidence for tactical deception. Behavioral Ecology and Sociobiology:1-11

Packer C (1979) Male dominance and reproductive activity in Papio anubis. Animal Behaviour 27:37-45

Packer C, Herbst L, Pusey AE, Bygott JD, Hanby JP, Cairns SJ, Borgerhoff-Mulder M (1988) Reproductive success of lions. In: Clutton-Brock TH (ed) Reproductive success. University of Chicago Press, Chicago, pp 363-383

Packer C, Pusey AE (1982) Cooperation and compatition within coalitions of male lions: kin selection or game theory? Nature 296:740-742

Palombit RA, Cheney DL, Fischer J, Johnson S, Rendall D, Seyfarth RM, Silk JB (2000) Male infanticide and defense of infants in chacma baboons. In: van Schaik $\mathrm{CP}$, Janson $\mathrm{CH}$ (eds) Infanticide by males and its implications. Cambridge University Press, Cambridge, pp 123-152

Palombit RA, Seyfarth RM, Cheney DL (1997) The adaptive value of 'friendships' to female baboons: experimental and observational evidence. Animal Behaviour 54:599-614

Pandit S, van Schaik C (2003) A model for leveling coalitions among primate males: toward a theory of egalitarianism. Behavioral Ecology and Sociobiology 55:161-168

Paul A (1989) Determinants of male mating success in a large group of Barbary macaques (Macaca sylvanus) at Affenberg Salem. Primates 30:461-476

Paul A, Kuester J (1988) Life-history patterns of Barbary macaques (Macaca sylvanus) at Affenberg Salem. In: Fa JE, Southwick CH (eds) Ecology and behavior of food-enhanced primate groups. Alan R. Liss, New York, pp 199-228

Paul A, Kuester J, Arnemann J (1996) The sociobiology of male-infant interactions in barbary macaques, Macaca sylvanus. Animal Behaviour 51:155-170

Pelletier F, Mainguy J, Côté SD (2009) Rut-induced hypophagia in male bighorn sheep and mountain goats: foraging under time budget constraints. Ethology 115:141-151

Perry S (1998) Male-male social relationships in wild white-faced capuchins, Cebus capucinus. Behaviour 135:139- 172

Perry S (2012) The behavior of wild white-faced capuchins: demography, life history, social relationships, and communication. Advances in the Study of Behavior 44:135-181

Perry S, Barrett H, Manson J (2004) White-faced capuchin monkeys show triadic awareness in their choice of allies. Animal Behaviour 67:165-170

Pfefferle D, Heistermann M, Pirow R, Hodges JK, Fischer J (2011) Estrogen and progestogen correlates of the structure of female copulation calls in semi-free-ranging Barbary macaques (Macaca sylvanus). International Journal of Primatology 32:992-1006

Plavcan J (2004) Sexual selection, measures of sexual selection, and sexual dimorphism in primates. In: Kappeler $P$, van Schaik $C$ (eds) Sexual selection in primates - new and comparative perspectives. Cambridge University Press, Cambridge, pp 230-252

Plavcan J, van Schaik C (1992) Intrasexual competition and canine dimorphism in anthropoid primates. American Journal of Physical Antropology 87:461-477

Plavcan JM, van Schaik CP (1997) Intrasexual competition and body weight dimorphism in anthropoid primates. American Journal of Physical Anthropology 103:37-68

Poole JH (1989) Mate guarding, reproductive success and female choice in African elephants. Animal Behaviour 37, Part 5:842-849

Port M, Johnstone RA, Kappeler PM (2010) Costs and benefits of multi-male associations in redfronted lemurs (Eulemur fulvus rufus). Biology Letters 6:620-622 
Port M, Johnstone RA, Kappeler PM (2012) The evolution of multimale groups in Verreaux's sifaka, or how to test an evolutionary demographic model. Behavioral Ecology 23:889897

Port M, Kappeler PM (2010) The utility of reproductive skew models in the study of male primates, a critical evaluation. Evolutionary Anthropology 19:46-56

Preston BT, Stevenson IR, Pemberton JM, Coltman DW, Wilson K (2003) Overt and covert competition in a promiscuous mammal: the importance of weaponry and testes size to male reproductive success. Proceedings of the Royal Society of London B: Biological Sciences 270:633-640

Puga-Gonzalez I, Hildenbrandt H, Hemelrijk CK (2009) Emergent patterns of social affiliation in primates, a model. PLoS Computational Biology 5:e1000630

Purvis A (1995) A composite estimate of primate phylogeny. Philosophical Transactions of the Royal Society, London, Series B 348:405-421

R Development Core Team (2011) R: A language and environment for statistical computing. In. R Foundation for Statistical Computing, Vienna, Austria

R Development Core Team (2012) R: A language and environment for statistical computing. R Foundation for Statistical Computing, Vienna, Austria. http://www.R-project.org/

Range F, Noe $R$ (2005) Can simple rules account for the pattern of triadic interactions in juvenile and adult female sooty mangabeys? Animal Behaviour 69:445-452

Rasmussen KLR (1985) Changes in the activity budgets of yellow baboons (Papio cynocephalus) during sexual consortships. Behavioral Ecology and Sociobiology 17:161-170

Rasmussen LEL, Schulte BA (1998) Chemical signals in the reproduction of Asian (Elephas maximus) and African (Loxodonta africana) elephants. Animal Reproduction Science 53:19-34

Reichert KE, Heistermann M, Hodges JK, Boesch C, Hohmann G (2002) What females tell males about their reproductive status: are morphological and behavioural cues reliable signals of ovulation in bonobos (Pan paniscus)? Ethology 108:583-600

Riss D, Goodall J (1977) The recent rise to the alpha-rank in a population of free-living chimpanzees. Folia Primatologica 27:134- 151

Say L, Pontier D, Natoli E (2001) Influence of oestrus synchronization on male reproductive success in the domestic cat (Felis catus L.). Proceedings of the Royal Society of London. Series B: Biological Sciences 268:1049-1053

Schilder M, B., H. (1990) Interventions in a herd of semi-captive plains zebras. Behaviour 112:5383

Schino G (2007) Grooming and agonistic support: a meta-analysis of primate reciprocal altruism. Behavioral Ecology 18 115-120

Schino G, Aureli F (2009) Reciprocal altruism in primates: partner choice, cognition, and emotions. Advances in the Study of Behavior 39:45-69

Schino G, Aureli F (2010) Primate reciprocity and its cognitive requirements. Evolutionary Anthropology 19:130-135

Schino G, Tiddi B, Polizzi Di Sorrentino E (2006) Simultaneous classification by rank and kinship in Japanese macaques. Animal Behaviour 71:1069-1074

Schubert M, Schradin C, Rödel HG, Pillay N, Ribble DO (2009) Male mate guarding in a socially monogamous mammal, the round-eared sengi: on costs and trade-offs. Behavioral Ecology and Sociobiology 64:257-264

Schülke O, Bhagavatula J, Vigilant L, Ostner J (2010) Social bonds enhance reproductive success in male macaques. Current Biology 220:2207-2210

Schülke O, Ostner J (2012) Ecological and social influences on sociality. In: Mitani JC, Call J, Kappeler PM, Palombit RA, Silk JB (eds) The evolution of primate societies. The University of Chicago Press, Chicago, pp 195-219

Seltmann A, Majolo B, Schülke O, Ostner J (2013) The organization of collective group movements in wild Barbary macaques (Macaca sylvanus): social structure drives processes of group coordination in macaques. PLoS ONE 8:e67285 
Semple S (1998) The function of Barbary macaque copulation calls. Proceedings of the Royal Society London B 265:287-291

Setchell J, Charpentier M, Wickings E (2005) Mate guarding and paternity in mandrills: factors influencing alpha male monopoly. Animal Behaviour 70:1105-1120

Setchell J, Wickings E (2005) Dominance, status signals and coloration in male mandrills (Mandrillus sphinx). Ethology 111:25-50

Setchell JM (2003) The evolution of alternative reproductive morphs in male primates. In: Jones C (ed) Special Topics in Primatology, vol 3. American Society of Primatologists, Norman, Oklahoma, pp 413-435

Setchell JM, Huchard E (2012) The hidden benefits of sex: evidence for MHC-associated mate choice in primate societies. Bioessays 32:940-948

Shelmidine N, Borries C, Koenig A (2007) Genital swellings in silvered langurs: what do they indicate? American Journal of Primatology 69:519-532

Sherman PW (1989) Mate guarding as paternity insurance in Idaho ground squirrels. Nature 338:418-420

Shideler SE, Ortuño AM, Morán FM, Moorman EA, Lasley BL (1993) Simple extraction and enzyme immunoassays for estrogen and progesterone metabolites in the feces of Macaca fascicularis during non-conceptive and conceptive ovarian cycles. Biology of Reproduction 48:1290-1298

Shively C, Smith D (1985) Social status and reproductive success of male Macaca fascicularis. American Journal of Primatology 9:129-135

Silk JB (1992) The patterning of intervention among male bonnet macaques: reciprocity, revenge, and loyalty. Current Anthropology 33:318-325

Silk JB (1994) Social relationships of male bonnet macaques: male bonding in a matrilineal society. Behaviour 130:271-291

Silk JB (1999) Male bonnet macaques use information about third-party rank relationships to recruit allies. Animal Behaviour 58:45-51

Silk JB (2002a) Kin selection in primate groups. International Journal of Primatology 23:849-875

Silk JB (2002b) Practice random acts of aggression and senseless acts of intimidation: the logic of status contests in social groups. Evolutionary Anthropology 11:221-225

Silk JB (2002c) Using the 'F'-word in primatology. Behaviour:421-446

Silk JB (2003) Social bonds of female baboons enhance infant survival. Science 302:1231-1234

Silk JB (2005) The evolution of cooperation in primate groups. In: Gintis H, Bowles R (eds) Moral Sentiments and Material Interests - The Foundation of Cooperation in Economic Life. MIT Press, Cambridge, Massachussetts pp 43-73

Silk JB (2007) The adaptive value of sociality in mammalian groups. Philosophical Transactions of the Royal Society B: Biological Sciences 362:539-559

Silk JB (2012) The adaptive value of sociality. In: Mitani J, C, Call J, Kappeler P, M., Palombit R, A., Silk JB (eds) The evolution of primate societies. The University of Chicago Press, Chicago, pp 552-564

Silk JB, Alberts SC, Altmann J (2006a) Social relationships among adult female baboons (Papio cynocephalus) II. Variation in the quality and stability of social bonds. Behavioral Ecology and Sociobiology 61:197-204

Silk JB, Altmann J, Alberts SC (2006b) Social relationships among adult female baboons (Papio cynocephalus) I. Variation in the strength of social bonds. Behavioral Ecology and Sociobiology 6:183-195

Silk JB, Beehner J, Bergman T, Crockford C, Engh A, Moscovice L, Wittig R, Seyfarth RM, Cheney DL (2010a) Female chacma baboons form strong, equitable, and enduring social bonds. Behavioral Ecology and Sociobiology 64:1733-1747

Silk JB, Beehner J, Bergman T, Crockford C, Engh A, Moscovice L, Wittig R, Seyfarth RM, Cheney DL (2010b) Strong and consistent social bonds enhance the longevity of female baboons. Current Biology 20:1359-1361 
Silk JB, Beehner JC, Bergman TJ, Crockford C, Engh AL, Moscovice LR, Wittig RM, Seyfarth RM, Cheney DL (2009) The benefits of social captial: close social bonds among female baboons enhance offspring survival. Proceedings of the Royal Society B 276:3099-3104

Small M (1990) Promiscuity in Barbary Macaques (Macaca sy/vanus). American Journal of Primatology 20:267-282

Smith JE, Van Horn RC, Powning KS, Cole AR, Graham KE, Memenis SK, Holekamp KE (2010) Evolutionary forces favoring intragroup coalitions among spotted hyenas and other animals. Behavioral Ecology 21:284-303

Snyder-Mackler N, Alberts SC, Bergman TJ (2012) Concessions of an alpha male? Cooperative defence and shared reproduction in multi-male primate groups. Proceedings of the Royal Society B: Biological Sciences 279:3788-3795

Soltis J, Mitsunaga F, Shimizu K, Nozakis M, Yanagihara Y, Domingo-Roura X, Takenaka O (1997) Sexual selection in Japanese macaques II: female mate choice and male-male competition. Animal Behaviour 54:737-746

Soltis J, Thomsen R, Takenaka $O$ (2001) The interaction of male and female reproductive strategies and paternity in wild Japanese macaques, Macaca fuscata. Animal Behaviour 62:485-494

Stamatopoulos G, Sengupta A, Vogel E, Janson C (2009) A game-theoretic model of coalition formation among primates. Journal of Bioeconomics 11:165-183

Sterck EHM, Watts DP, van Schaik CP (1997) The evolution of female social relationships in nonhuman primates. Behavioral Ecology and Sociobiology 41:291-309

Stevens JR, Cushman FA, Hauser MD (2005) Evolving the psychological mechanisms for cooperation. Annual Review of Ecology, Evolution, and Systematics 36:499-518

Stevens JR, Hauser MD (2004) Why be nice? Psychological constraints on the evolution of cooperation. Trends in Cognitive Sciences 8:60-65

Strier KB (1994) Brotherhoods among atelins: kinship, affiliation, and competition. Behaviour 130:151-167

Strier KB (1996) Male reproductive strategies in new world primates. Human Nature 7:105-123

Strier KB, Chaves PB, Mendes SrL, Fagundes Vr, Di Fiore A (2011) Low paternity skew and the influence of maternal kin in an egalitarian, patrilocal primate. Proceedings of the National Academy of Sciences 108:18915-18919

Sukmak M, Wajjwalku W, Schülke O, Ostner J (submitted) The Priority-of-Access-Model predicts paternity distribution in wild male Assamese macaques. Behavioral Ecology and Sociobiology

Swaisgood RR, Lindburg DG, Zhang H (2002) Discrimination of oestrous status in giant pandas (Ailuropoda melanoleuca) via chemical cues in urine. Journal of Zoology 257:381-386

Takahashi H (2004) Do males have a better chance of mating when the number of estrous females is equal to or greater than the males' ordinal rank? Testing the hypothesis in Japanese macaques. American Journal of Primatology 63:95-102

Taub D (1980) Female choice and mating strategies among wild Barbary macaques (Macaca sylvanus L.). In: Lindburg D (ed) The Macaques: Studies in Ecology. Behavior and Evolution. Van Nostrand Reinhold Co., New York, pp 287-344

Teichroeb JA, Wikberg EC, Ting N, Sicotte P (2013) Factors influencing male affiliation and coalitions in a species with male dispersal and intense male-male competition, Colobus vellerosus. In: Behaviour, pp 1-22

Trivers RL (1971) The evolution of reciprocal altruism. The Quarterly Review of Biology 46:35-57

Trivers RL (1972) Parental investment and sexual selection. In: Campbell B (ed) Sexual selection and the descent of man, 1871-1971. Heinemann, London, pp 136-179

van Hooff JARAM, van Schaik CP (1992) Cooperation in competition: the ecology of primate bonds. In: Harcourt AH, de Waal FBM (eds) Coalitions and alliances in humans and other animals. Oxford University Press, Oxford, pp 357-389

van Hooff JARAM, van Schaik CP (1994) Male bonds: affiliative relationships among nonhuman primate males. Behaviour 130:309-337 
van Noordwijk MA, van Schaik CP (2004) Sexual selection and the careers of primate males: paternity concentration, dominance-acquisition tactics and transfer decisions. In: Kappeler PM, van Schaik CP (eds) Sexual selection in primates - new and comparative perspectives. Cambridge University Press, Cambridge, pp 208-229

van Schaik C, Hodges J, Nunn C (2000) Paternity confusion and the ovarian cycles of female primates. In: van Schaik C, Janson C (eds) Infanticide by males and its implications. Cambridge University Press, Cambridge, pp 361-387

van Schaik C, Pandit S, Vogel E (2004a) A model for within-group coalitionary aggression among males. Behavioral Ecology and Sociobiology 57:101-109

van Schaik C, Pandit S, Vogel E (2006) Toward a general model for male-male coalitions in primate groups. In: Kappeler $\mathrm{P}$, van Schaik $\mathrm{C}$ (eds) Cooperation in primates and humans. Springer, Heidelberg, pp 151-172

van Schaik CP (1989) The ecology of social relationships amongst female primates. In: Standen V, Foley RA (eds) Comparative socioecology. The behavioural ecology of humans and other mammals. Blackwell Scientific Publications, Oxford, pp 195-218

van Schaik CP, Pradhan GR, van Noordwijk MA (2004b) Mating conflict in primates: infanticide, sexual harrassment and female sexuality. In: Kappeler PM, van Schaik CP (eds) Sexual selection in primates: New and comparative perspectives. Cambridge University Press, Cambridge, pp 131-150

Vigilant L, Hofreiter M, Siedel H, Boesch C (2001) Paternity and relatedness in wild chimpanzee communities. Proceedings of the National Academy of Science 98:12890-12895

Wallen K (2001) Sex and context: hormones and primate sexual motivation. Hormones and Behavior 40:339-357

Wallen K, Winston LA, Gaventa S, Davis-DaSilva M, Collins DC (1984) Periovulatory changes in female sexual behavior and patterns of ovarian steroid secretion in group-living rhesus monkeys. Hormones and Behavior 18:431-50

Wallner B, Möstl E, Dittami J, Prossinger H (1999) Fecal glucocorticoids document stress in female Barbary macaques (Macaca sylvanus). General and Comparative Endocrinology 113:80-86

Waser PM, Keane B, Creel SR, Elliott LF, Minchella DJ (1994) Possible male coalitions in a solitary mongoose. Animal Behaviour 47:289-294

Waterman JM (1997) Why do male Cape ground squirrels live in groups? Animal Behaviour 53:809-817

Watts DP (2002) Reciprocity and Interchange in the Social Relationships of Wild Male Chimpanzees. Behaviour 139:343- 370

Watts DP (2010) Dominance, power, and politics in nonhuman and human primates. In: Kappeler PM, Silk JB (eds) Mind the Gap: tracting the origins of human universals. Springer, Berlin, pp 109-138

Weidt A, Hofmann SE, König B (2008) Not only mate choice matters: fitness consequences of social partner choice in female house mice. Animal Behaviour 75:801-808

Weingrill T (2000) Infanticide and the value of male-female relationships in mountain chacma baboons. In: Behaviour, pp 337-359

Weingrill T, Lycett JE, Barrett L, Hill RA, Henzi SP (2003) Male consortship behaviour in chacma baboons: the role of demographic factors and female conceptive probabilities. Behaviour 140:405-427

Weingrill T, Lycett JE, Henzi SP (2000) Consortship and mating success in chacma baboons (Papio cynocephalus ursinus). Ethology 106:1033-1044

West S, Griffin A, Gardner A (2007a) Social semantics: altruism, cooperation, mutualism, strong reciprocity and group selection. Journal of Evolutionary Biology 20:415-432

West SA, Griffin AS, Gardner A (2007b) Evolutionary explanations for cooperation. Current Biology 17:R661-R672

Westlin LM (1996) Behavioural manifestation of conception 12 hours after mating in an asocial African rodent, Saccostomus campestris. Journal of Zoology 239:515-523 
Wey T, Blumstein D (2012) Social attributes and associated performance measures in marmots: bigger male bullies and weakly affiliating females have higher annual reproductive success. Behavioral Ecology and Sociobiology 66:1075-1085

Whitehead H, Connor R (2005) Alliances I. How large should alliances be? Animal Behaviour 69:117-126

Widdig A, Bercovitch F, Streich W, Sauermann U, Nürnberg P, Krawczak M (2004) A longitudinal analysis of reproductive skew in male rhesus macaques. Proceedings of the Royal Society London B 271:819-826

Widdig A, Streich WJ, Tembrock G (2000) Coalition formation among male Barbary macaques (Macaca sylvanus). American Journal of Primatology 50:37-51

Wilcox AJ, Weinberg CR, Baird DD (1995) Timing of sexual intercourse in relation to ovulation. New England Journal of Medicine 333:1517-1521

Wilson ME, Gordon TP, Collins DC (1982) Serum 17 beta-estradiol and progesterone associated with mating behavior during early pregnancy in female rhesus monkeys. Hormones and Behavior 16:94-106

Wittig RM, Crockford C, Lehmann J, Whitten PL, Seyfarth RM, Cheney DL (2008) Focused grooming networks and stress alleviation in wild female baboons. Hormones and Behavior 54:170-177

Wrangham RW (1980) An ecological model of female-bonded primate groups. Behaviour 75:262300

Wroblewski EE, Murray CM, Keele BF, Schumacher-Stankey JC, Hahn BH, Pusey AE (2009) Male dominance rank and reproductive success in chimpanzees, Pan troglodytes schweinfurthii. Animal Behaviour 77:873-885

Xia D-P, Li J-H, Garber P, A., Matheson M, D., Sun B-H, Zhu Y (2013) Grooming reciprocity in male Tibetan macaques. American Journal of Primatology:DOI: 10.1002/ajp.22165

Young C, Hähndel S, Majolo B, Schülke O, Ostner J (2013a) Male coalitions and female behaviour affect male mating success independent of dominance rank and female receptive synchrony in wild Barbary macaques. Behavioral Ecology and Sociobiology:DOI: 10.1007/s00265-013-1577-7

Young C, Majolo B, Heistermann M, Schülke O, Ostner J (2013b) Male mating behaviour in relation to female sexual swellings, socio-sexual behaviour and hormonal changes in wild Barbary macaques. Hormones and Behavior 63:32-39

Young C, Schülke O, Ostner J (in revision) Beyond mutual tolerance - how males form coalitions against group rivals and the Pandit|van Schaik coalition model. Behaviour

Zabel C, Glickman S, Frank L, Woodmansee K, Keppel G (1992) Coalition formation in a colony of prepubertal spotted hyaenas. In: Harcourt A, De Waal F (eds) Coalitions and alliances in humans and other animals. Oxford University Press, Oxford, pp 113-136

Zeh JA, Zeh DW (2001) Reproductive mode and the genetic benefits of polyandry. Animal Behaviour:1051-1063

Zehr JL, Maestripieri D, Wallen K (1998) Estradiol increases female sexual initiation independent of male responsiveness in rhesus monkeys. Hormones and Behaviour 33:95-103

Zehr JL, Tannenbaum PL, Jones B, Wallen K (2000) Peak occurrence of female sexual initiation predicts day of conception in rhesus monkeys (Macaca mulatta). Reproduction Fertility and Development 12:397-404 


\section{Acknowledgements}

Many people have provided me with help, support and advice throughout the duration of this thesis any I would show my appreciation to you all. First and foremost, I am eternally grateful to my supervisor team during my PhD studies. Julia Ostner, Oliver Schülke and Bino Majolo have all provided me with sage advice, great supervision and astute evaluation of manuscripts. Thank you for all your support, guidance and putting faith in me to succeed in this project.

I am thankful to Peter Kappeler for his support as the co-supervisor of my thesis. I further thank Peter Kappeler, Julia Fischer, Antje Engelhardt, Eckhard Heymann, and Dirk Semmann for agreeing to be members of my evaluation committee.

I am grateful to Professor Mohamed Qarro (Ecole Nationale Forestière d'Ingénieurs, Morocco) for his support in the field and the Haut Commissariat aux Eaux et Forêts et à la Lutte Contre la Désertification of Morocco for research permission. I would like to thank the Rakkas family who did so much for me in Morocco and who integrated me into a different culture with great food, welcoming attitudes and mint tea. Also to everyone in Azrou who made me feel a big part of the community, Šukran bezzaf (بـ زاف شـكرا). Additionally, to Rich who convinced me to join him in Morocco. The Ain Kahla forest provided us with some great memories and you provided important and useful advice along the way, thank you.

I am eternally grateful to my field team in Morocco; to say this project would not have been possible without you all is an understatement. You all did such a sterling job under great mental and physical pressures. Michael, Dave, Sofia, Maria, Tom and Josephine you suffered through some tough environmental conditions from sun and heat, to hail, to rain, to thunder to snow but your positive attitudes shone through. Thank you not only for the great work ethic and dedication but for sharing many great social experiences as well. An additionally I would like to show my appreciation to all the other PhD, Masters and research team members who were in Morocco during my time there, the constantly changing dynamics and new faces really added depth and enjoyment to my field time. I would also like to thank Sabine for her hard work and data collection to continue the project after I left the field.

I would also like to thank my collaborators and those who provided additional assistance. Firstly, I would like to show my gratitude to Michael Heistermann for providing input, training and guidance with endocrine analysis and interpretation. Additionally, I would like to thank Andrea Heistermann and Petra Kiesel for providing assistance and expertise during hormone analysis. I 
would like to take this opportunity to thank the Max Planck Society, the Christian Vogel Fonds and Georg-August University Göttingen through funds from the German Initiative of Excellence for financial support.

In my research group I have received great support from its ever changing composition and to all those who have shared my affiliation I thank you for support, critique and invaluable discussions. The group provided a great platform with which to discuss my ideas and conduct my research. I am grateful to Josi, Andreas and Annie for discussions on coalitions. A big thank you goes to Christiane Fuhlrott and Clarissa Licht for constantly providing me with assistance of German form filling and document translating both in Germany and Morocco, without which I would have been very lost. In a similar vein I thank Frederic and Pascal who helped with translation of my summary (zusammenfassung) to German.

My statistical knowledge would never have been as advanced as it is today if it were not for Christof and Cédric sharing and discussing information on loops, models, scripts, randomness, distributions and so much more, thank you guys. Additionally, I thank Cédric for many long discussions and debates about reproductive strategies and tactics and always making his point.

Göttingen provides a fairly unique environment for PhD students in Primatology with many different research groups clustered around the DPZ/CRC. To all those many Bachelor, Masters, $\mathrm{PhD}$ and Post-Doc's who have been here over the last few years thank you all for providing many good times. I think none of us will fully appreciate the unique dynamic provided with here until we have all moved on to pastures new. In particular I would like to thank Cédric, Christof, Gisela, Ines, Julie, Matthis, Marlies, Miranda, Pascal, Rebecca, Sally, Tabby for sharing so many great experiences and memories from Bond's, to bars to BBQ's and always tolerating the humour no matter the depths it reached.

I would especially like to acknowledge Tabby and Rebecca for expressing a willingness to exchange food for support and sharing many a weekend lunch together breaking up the writing melon-choly with fruitful discussions. Additionally, along with Julie they have provided a wonderful wee thesis support group, thank you guys.

I would like to give a special thank you to all my family and friends for providing unwavering support whilst I have been away in many foreign lands. Sometimes my work became my life and I thank you for understanding that. Finally a massive thank you to Laëtitia for sharing many great times in Morocco and beyond, helping out with field work when on holiday, for providing dependable support, enthusiasm and encouragement throughout. I am sure I shall be reciprocating all that very soon. 


\section{Curriculum Vitae}

Name: Christopher John Young

Place and date of birth: Dundee, U.K. $19^{\text {th }}$ October 1982

Nationality: British

\section{Education}

2009 - Present

Georg-August-Universität Göttingen

PhD Studentship

Thesis title: Cooperation and competition in wild male Barbary macaques (Macaca sylvanus) in Morocco.

$2006-2007$

\section{University of Roehampton, UK}

MRes in Primatology

Includes a field-based research dissertation on "The effects of tourist pressure on stress and human-macaque interactions of the Barbary macaques of Gibraltar".

$2000-2004$

\section{University of Edinburgh, UK}

BSc Zoology (Hons)

\section{Publications}

Young, C., Majolo, B., Schülke, O. \& Ostner, J. (Submitted). Male social bonds predict partner choice in cooperative aggression in wild Barbary macaques. Animal Behaviour.

Young, C., Schülke, O., \& Ostner, J. (In revision). Beyond mutual tolerance - how males form coalitions against group rivals and the Pandit/van Schaik coalition model. Behaviour.

Thunström, M., Kuchenbuch, P., \& Young, C. (In revision). Concealing of facial expressions in a wild Barbary macaque (Macaca sylvanus). International Journal of Primatology.

Young, C., Hähndel, S., Majolo, B., Schülke, O., \& Ostner, J. (Online) Male coalitions, dominance rank and female preferences independently affect male mating success in wild Barbary macaques. Behavioral Ecology and Sociobiology, DOI: 10.1007/s00265-013-1577-7

Majolo, B., McFarland, R., Young, C., Qarro, M. (2013) The Effect of climatic factors on the activity budgets of the Barbary macaque (Macaca sylvanus). International Journal of Primatology, 34: 500-514.

Young, C., Majolo, B., Heistermann, M., Schülke, O. \& Ostner, J. (2013). Male mating behaviour in relation to female sexual swellings, socio-sexual behaviour and hormonal changes in wild Barbary macaques. Hormones and Behavior, 63: 32-39.

Young, C., Schülke, O., Ostner, J. \& Majolo, B. (2012). Consumption of unusual prey items in the Barbary macaque (Macaca sylvanus). African Primates, 7: 224-229. 


\section{Conference contributions}

Young, C., Hähndel, S., Majolo, B., Schülke, O. \& Ostner, J. Male coalitions and female behaviour predict deviations of male mating success from the predictions of the Priority of Access model. (Oral presentation PSGB Spring meeting, Lincoln, 2013).

Young, C., Majolo, B., Heistermann, M., Schülke, O. \& Ostner, J. Male Barbary macaques invest equally in sexual behaviour during fertile and post-conception anogenital swelling periods. (Oral presentation Gesellschaft für Primatologie, Hamburg, 2013).

Young, C., Majolo, B., Heistermann, M., Schülke, O. \& Ostner, J. Honest signals? Barbary macaque males cannot differentiate conception and post-conception female anogenital swellings. (Poster at PSGB Spring Meeting, London, 2012).

Young, C., Majolo, B., Schülke, O. \& Ostner, J. The flexibility of coalition formation in Barbary macaques. (Poster at Göttinger Freilandtage 2011).

\section{Research experience}

2008: $\quad$ Research Assistant for PhD student (Richard McFarland), University of Lincoln. A study on socio-ecology of wild Barbary macaques in Morocco.

2010-2012: Scientific advisor. BBC Africa, BBC Natural History Unit, Bristol, UK.

\section{External funding \research grants}

2011: Christian Vogel Fonds

Cooperation and competition in wild male Barbary macaques in Morocco

2010: Georg-August-Universität Göttingen

Cooperation and competition in wild male Barbary macaques in Morocco

2009: Max Planck Institute

Cooperation and competition in wild male Barbary macaques in Morocco

\section{Memberships and affiliations}

International Primatological Society

Primate Society of Great Britain

Gesellschaft für Primatologie e.V. 
I hereby declare that I have written this thesis entitled "Cooperation and competition in wild male Barbary macaques (Macaca sylvanus) in Morocco" independently and with no other aids or sources than quoted.

\section{Göttingen,}

Christopher Young 